\title{
MID-INFRARED PROPERTIES OF OH MEGAMASER HOST GALAXIES. I. SPITZER IRS LOW- AND HIGH-RESOLUTION SPECTROSCOPY
}

\author{
Kyle W. Willett ${ }^{1}$, Jeremy Darling ${ }^{1}$, Henrik W. W. Spoon ${ }^{2}$, Vassilis Charmandaris $^{3,4,5}$, and Lee Armus ${ }^{6}$ \\ ${ }^{1}$ Center for Astrophysics and Space Astronomy, Department of Astrophysical and Planetary Sciences, UCB 391, \\ University of Colorado, Boulder, CO 80309-0391, USA; willettk@ colorado.edu \\ ${ }^{2}$ Astronomy Department, Cornell University, Ithaca, NY 14853, USA \\ ${ }^{3}$ Department of Physics and Institute of Theoretical \& Computational Physics, University of Crete, GR-71003 Heraklion, Greece \\ ${ }^{4}$ IESL/Foundation for Research and Technology-Hellas, GR-71110 Heraklion, Greece \\ ${ }^{5}$ Chercheur Associé, Observatoire de Paris, F-75014 Paris, France \\ ${ }^{6}$ Spitzer Science Center, California Institute of Technology, Pasadena, CA 91125, USA \\ Received 2010 March 19; accepted 2011 January 26; published 2011 March 3
}

\begin{abstract}
We present mid-infrared spectra and photometry from the Infrared Spectrograph on the Spitzer Space Telescope for $51 \mathrm{OH}$ megamasers (OHMs), along with 15 galaxies confirmed to have no megamaser emission above $L_{\mathrm{OH}}=10^{2.3} L_{\odot}$. The majority of galaxies display moderate-to-deep $9.7 \mu \mathrm{m}$ amorphous silicate absorption, with OHM galaxies showing stronger average absorption and steeper 20-30 $\mu \mathrm{m}$ continuum emission than non-masing galaxies. Emission from multiple polycyclic aromatic hydrocarbons (PAHs), especially at 6.2, 7.7, and $11.3 \mu \mathrm{m}$, is detected in almost all systems. Fine-structure atomic emission (including [Ne II], [Ne III], [S III], and [S IV]) and multiple $\mathrm{H}_{2}$ rotational transitions are observed in more than $90 \%$ of the sample. A subset of galaxies show emission from rarer atomic lines, such as [Ne v], [O IV], and [Fe II]. Fifty percent of the OHMs show absorption from water ice and hydrogenated amorphous carbon grains, while absorption features from $\mathrm{CO}_{2}, \mathrm{HCN}, \mathrm{C}_{2} \mathrm{H}_{2}$, and crystalline silicates are also seen in several OHMs. Column densities of $\mathrm{OH}$ derived from $34.6 \mu \mathrm{m} \mathrm{OH}$ absorption are similar to those derived from $1667 \mathrm{MHz} \mathrm{OH}$ absorption in non-masing galaxies, indicating that the abundance of masing molecules is similar for both samples. This data paper presents full mid-infrared spectra for each galaxy, along with measurements of line fluxes and equivalent widths, absorption feature depths, and spectral indices.
\end{abstract}

Key words: galaxies: active - galaxies: starburst - infrared: galaxies - masers

Online-only material: extended figures

\section{INTRODUCTION}

OH megamasers (OHMs) are $18 \mathrm{~cm}$ masers with integrated line luminosities on the order of $10^{1}-10^{4} L_{\odot}$. They are an extremely rare phenomenon in the local universe, with roughly 100 currently known out to a redshift of $z=0.265$ (Baan et al. 1992). All OHMs, including the more powerful "gigamasers" $\left(L_{\mathrm{OH}}>10^{4} L_{\odot}\right)$ are associated with starburst nuclei in merging galaxies. OHMs have been identified in many different types of nuclear environments as classified by optical spectra, but the merging galaxies are without exception (ultra)luminous infrared galaxies ((U)LIRGs). Since OHMs are signposts of gas-rich merging galaxies, their presence can also indicate the existence of associated phenomena including massive black hole mergers and highly obscured circumnuclear starbursts (Darling 2007). OHMs are a powerful tool in this respect due in large part to their ability to be seen at cosmic distances. In order to employ OHMs as tracers, however, the assumption must be made that the $\mathrm{OH}$ line properties remain constant as a function of cosmic time and host environment. An explanation of the physical mechanisms and conditions responsible for distinguishing OHMs from non-masing ULIRGs is thus vital for understanding both the megamaser phenomenon and the associated merger characteristics.

Spectroscopic studies of the mid-IR emission in the host galaxies offer multiple diagnostics which can provide clues to the nature of the maser pumping mechanism and the associated $\mathrm{OH}$ emission. We used the Infrared Spectrograph (IRS) on board the Spitzer Space Telescope (Werner et al. 2004) to study merging ULIRGs. Since the dusty nuclear regions are typically obscured at optical wavelengths, mid-IR observations can yield valuable information specific to the locations in which the OHMs are generated. These include measurements of the dust temperature and optical depth (from broadband photometry and absorption features), the excitation and temperature of the gas (molecular and fine-structure atomic lines), and high-ionization lines that can signal the presence of an active galactic nucleus (AGN), a possible heating source for the dust.

This data paper presents full low-resolution (LR) and highresolution (HR) IRS spectra along with measured mid-IR properties for 51 OHMs and 15 non-masing ULIRGs. An accompanying paper (Willett et al. 2011, Paper II) presents the full analysis, statistical comparisons of the masing and nonmasing galaxies, and tests the viability of current OHM pumping models based on the IRS data.

\section{THE SAMPLE}

The OHM host galaxies selected for IRS observations were primarily drawn from the Arecibo OHM survey (Darling \& Giovanelli 2002a, 2002b). We selected well-studied OHMs with unambiguous maser detections and large amounts of ancillary data (including $\mathrm{OH}$ line and radio continuum maps, near-IR imaging, and optical imaging and spectroscopy) to maximize scientific return on the sample. A lower threshold of $L_{\mathrm{OH}}>10^{1.6} L_{\odot}$ also eliminated extragalactic "kilomasers" from the sample, which are likely powered by different radiative processes than megamasers (Henkel \& Wilson 1990).

In order to be detected in reasonable integration times using the IRS, we required that all potential targets have 
$S(60 \mu \mathrm{m})>0.8 \mathrm{Jy}$ as measured by the Infrared Astronomical Satellite (IRAS). After removing objects already observed by the IRS (largely through the GTO ULIRG program; e.g., Armus et al. 2007), we observed 24 galaxies in the redshift range $0.1<z<0.2$.

We supplemented these galaxies with additional spectra of OHM hosts publicly available through the Spitzer archive. To ensure uniformity of the data, we selected only galaxies from the archive that had full coverage with both the IRS LR and HR modules. As of 2008 March, the publicly available data from the archive yielded 27 additional OHM galaxies (Table 1).

In order to provide a baseline for analysis of the OHMs, we also identified a control sample of ULIRGs that showed no megamaser emission above a firm limit. To identify these galaxies, we drew on non-detections from $\mathrm{OH}$ surveys by Baan et al. (1992), Staveley-Smith et al. (1992), Darling \& Giovanelli (2000, 2001, 2002a), and Kent et al. (2002). The upper limit for $\mathrm{OH}$ emission is conservatively derived from the rms noise in the spectrum at $1667 \mathrm{MHz}$, assuming a boxcar line profile with a line width $\Delta v=150 \mathrm{~km} \mathrm{~s}^{-1}$ and a $1.5 \sigma$ detection:

$$
L_{\mathrm{OH}}^{\max }=4 \pi D_{L}^{2}(1.5 \sigma)\left(\frac{\Delta v}{c}\right)\left(\frac{v_{0}}{1+z}\right) .
$$

For this control sample, we set an upper limit of $L_{\mathrm{OH}}^{\max }<$ $10^{2.3} L_{\odot}$; this limit compromises between ensuring that all but the faintest megamaser emission is excluded and yielding a reasonable number of objects in the control sample for statistical analysis. All $51 \mathrm{OHMs}$ in our IRS sample have $L_{\mathrm{OH}}$ above this limit.

In addition to selecting galaxies based on $\mathrm{OH}$ non-detection, we imposed two additional criteria to ensure that the control sample was as similar as possible to the OHM hosts. First, we set a lower limit on the far-infrared luminosity (Sanders \& Mirabel 1996) of the non-masing galaxies as measured by their IRAS fluxes. OHMs occur exclusively in IR-bright galaxies, due to the fact that the maser is pumped primarily by rotational transitions of a few hundred kelvin above the ground state (Baan et al. 1982; Henkel et al. 1987). Darling \& Giovanelli (2002a) show that the relationship between the $\mathrm{OH}$ and infrared luminosities is a power law with $L_{\mathrm{OH}} \propto L_{\mathrm{FIR}}^{1.2}$. Since no OHM observed with the IRS has $L_{\mathrm{FIR}}<10^{11} L_{\odot}$, we established this as the lower limit for inclusion in the non-masing sample.

Second, a cutoff in redshift space is applied to sample a sufficiently large volume $\left(V \sim 1 \mathrm{Gpc}^{3}\right)$ in order to avoid systematic effects such as the Malmquist bias. The available data in the archive contained many more galaxies at lower redshifts $(z<0.05)$ than those further away. To avoid overweighting the control sample toward galaxies at low redshifts, we sorted galaxies that met the $L_{\mathrm{OH}}^{\max }$ and $L_{\mathrm{IR}}$ criteria into bins of $\Delta z=$ 0.02 . For bins where the number of non-masing galaxies exceeded those of OHMs, we randomly removed objects from the non-masing bins until the numbers were equal. This reduced the control sample to one galaxy at $0<z<0.02$ and four galaxies at $0.02<z<0.04$; for all other redshift bins, no such adjustments were necessary. As of 2008 March, there existed 15 suitable candidates in the Spitzer archive qualifying for the nonmasing control sample (Table 1). Figure 1 shows the distribution of $\mathrm{OH}$ luminosity for all objects as a function of redshift; for the non-masing galaxies, we display upper limits as computed in Equation (1).

Throughout this paper, we assume the WMAP5 cosmology with $H_{0}=70.5, \Omega_{M}=0.274$, and $\Omega_{\Lambda}=0.726$ (Hinshaw et al. 2009).

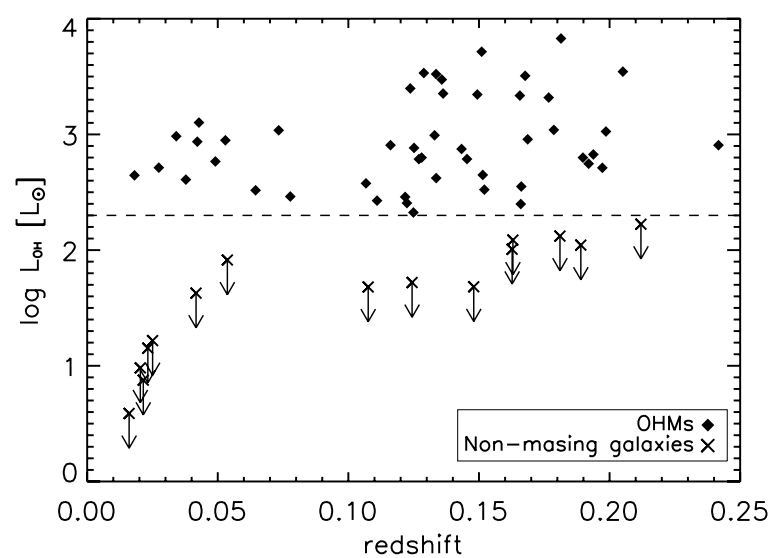

Figure 1. Distribution of integrated $\mathrm{OH}$ luminosity for the IRS samples as a function of redshift. For non-masing galaxies, the $\mathrm{OH}$ luminosity is an upper limit calculated from Equation (1). The dashed line at $L_{\mathrm{OH}}=10^{2.3} L_{\odot}$ is the upper limit on possible $\mathrm{OH}$ emission for the non-masing control sample.

\section{OBSERVATIONS}

We observed 24 OHMs with the IRS from 2006 August through 2007 December. The IRS contains four modules in two different spectroscopic resolutions: LR and HR (Houck et al. 2004). The short-high (SH) and long-high (LH) modules operate at a resolution of $R \sim 600$; the short-low (SL1 and SL2) and long-low (LL1 and LL2) modules operate at resolutions ranging from $R \sim 56-127$, depending on the observing wavelength. Two orders, SL3 $(7.3-8.7 \mu \mathrm{m})$ and LL3 $(19.4-21.7 \mu \mathrm{m})$, cover the overlapping range between the first and second orders in both SL and LL. We used these only as checks for the absolute flux calibration between the different orders.

All targets used the Staring Mode Astronomical Observing Template (AOT) with the galaxies placed at two nod positions approximately one-third and two-thirds the length of the slit. We observed targets in all six modules as well as an equaltime, off-target exposure in the LH module to be used for sky subtraction.

Although the majority of our sources had no previous IRAS detections in either the 12 or $25 \mu \mathrm{m}$ bands, we extrapolated the likely flux based on the colors of ULIRGs at similar redshifts and the measured fluxes at $60 \mu \mathrm{m}$. We chose cycle times intended to yield signal-to-noise ratios of $\mathrm{S} / \mathrm{N}=50-100$ for the $\mathrm{LR}$ modules and $\mathrm{S} / \mathrm{N} \geqslant 10$ for the HR modules, allowing for accurate measurement of faint emission and absorption features, as well as accurate spectral decomposition.

For the 24 objects in the dedicated OHM program, we took dedicated peakup observations in both the blue $(16 \mu \mathrm{m})$ and red $(22 \mu \mathrm{m})$ filters in the sample-up-the-ramp (SUR) mode for photometric calibration of the spectra. The SUR peakups did not coincide in time with the spectroscopy for the majority of targets. None of the galaxies selected from the Spitzer archive possessed SUR peakup data; 16 archived galaxies have double correlated sampling (DCS) peakups with slightly worse photometric accuracy than those with SUR data. Only one galaxy, IRAS 10173+0828, had no peakup observations of any kind. Measured peakup fluxes are given in Table 3.

The Spitzer beam is diffraction limited past $6 \mu \mathrm{m}$, with slit widths for the spectral modules between $3^{\prime \prime}$ and $11^{\prime \prime}$. The vast majority of the nuclei in merging ULIRGs have angular separations less than the instrument point-source function, and thus can be treated as point sources. For the few galaxies close 
Table 1

Radio, Optical, and FIR Properties of OHMs and Non-masing Galaxies

\begin{tabular}{|c|c|c|c|c|c|c|c|c|c|}
\hline & IRAS FSC & $\begin{array}{c}\text { R.A. } \\
\text { J2000.0 }\end{array}$ & $\begin{array}{c}\text { Decl. } \\
\text { J2000.0 }\end{array}$ & $z_{\odot}^{\mathrm{a}}$ & $\begin{array}{c}D_{L} \\
\left(h_{70}^{-1} \mathrm{Mpc}\right)\end{array}$ & $\begin{array}{c}\log L_{\mathrm{FIR}}{ }^{\mathrm{b}} \\
\left(h_{70}^{-2} L_{\odot}\right)\end{array}$ & $\begin{array}{l}\log L_{\mathrm{OH}}{ }^{\mathrm{c}} \\
\left(h_{70}^{-2} L_{\odot}\right)\end{array}$ & $\begin{array}{c}f_{1.4 \mathrm{GHz}}^{\mathrm{d}} \\
(\mathrm{mJy})\end{array}$ & Alt. Desig. \\
\hline \multirow[t]{51}{*}{ OHMs } & IRAS $01355-1814$ & 013757.4 & -175921 & 0.191 & 929 & 12.49 & 2.75 & $<5.0$ & \\
\hline & IRAS $01418+1651$ & 014430.5 & +170605 & 0.0274 & 115 & 11.63 & 2.71 & 40.6 & III Zw 035 \\
\hline & IRAS $01562+2528$ & 015902.6 & +254237 & 0.1658 & 788 & 12.19 & 3.31 & 6.3 & \\
\hline & IRAS $02524+2046$ & 025517.1 & +205843 & 0.1815 & 873 & $12.07-12.54$ & 3.80 & 2.9 & \\
\hline & IRAS $03521+0028$ & 035442.2 & +003703 & 0.1522 & 718 & 12.59 & 2.49 & 6.7 & \\
\hline & IRAS $04121+0223$ & 041447.1 & +023036 & 0.1216 & 568 & $11.69-11.96$ & 2.39 & 3.1 & \\
\hline & IRAS 04454-4838 & 044649.5 & -483333 & 0.0529 & 235 & 11.89 & 2.95 & $<5.0$ & ESO 203-IG 001 \\
\hline & IRAS $06487+2208$ & 065145.8 & +220427 & 0.1437 & 678 & 12.34 & 2.87 & 10.8 & \\
\hline & IRAS $07163+0817$ & 071905.5 & +08 1207 & 0.1107 & 515 & 11.79 & 2.43 & 3.5 & \\
\hline & IRAS $07572+0533$ & 075957.2 & +052500 & 0.1894 & 926 & 12.31 & 2.80 & 5.0 & \\
\hline & IRAS $08201+2801$ & 082312.6 & +275140 & 0.1680 & 808 & 12.26 & 3.51 & 16.7 & \\
\hline & IRAS $08449+2332$ & 084751.0 & +232106 & 0.1510 & 723 & 12.05 & 2.65 & 6.1 & \\
\hline & IRAS $08474+1813$ & 085018.3 & +180201 & 0.1450 & 692 & 12.19 & 2.76 & 4.2 & \\
\hline & IRAS 09039+0503 & 090634.2 & +045125 & 0.1250 & 589 & 12.16 & 2.88 & 6.6 & \\
\hline & IRAS 09539+0857 & 095634.3 & +084306 & 0.1290 & 608 & 12.09 & 3.53 & 9.5 & \\
\hline & IRAS $10035+2740$ & 100626.3 & +272546 & 0.1662 & 794 & 12.26 & 2.55 & 6.3 & \\
\hline & IRAS $10039-3338$ & 100604.8 & -335315 & 0.0341 & 154 & 11.74 & 2.98 & 24.7 & \\
\hline & IRAS $10173+0828$ & 102000.2 & +08 1334 & 0.0480 & 222 & 11.86 & 2.77 & 10.8 & \\
\hline & IRAS $10339+1548$ & 103637.9 & +153242 & 0.1965 & 969 & 12.35 & 2.71 & 5.1 & \\
\hline & IRAS $10378+1109$ & 104029.2 & +105318 & 0.1362 & 646 & 12.35 & 3.35 & 8.9 & \\
\hline & IRAS $10485-1447$ & 105103.1 & -150322 & 0.1330 & 629 & 12.23 & 2.99 & 4.4 & \\
\hline & IRAS $11028+3130$ & 110537.5 & +311432 & 0.1990 & 975 & 12.39 & 3.03 & 5.0 & \\
\hline & IRAS $11180+1623$ & 112041.7 & +160657 & 0.1660 & 801 & 12.27 & 2.40 & 4.2 & \\
\hline & IRAS $11524+1058$ & 115502.8 & +104144 & 0.1784 & 868 & 12.19 & 3.04 & 5.0 & \\
\hline & IRAS 12018+1941 & 120424.5 & +192510 & 0.1687 & 814 & 12.48 & 2.96 & 6.5 & \\
\hline & IRAS $12032+1707$ & 120547.7 & +165108 & 0.2170 & 1082 & 12.64 & 4.21 & 28.7 & \\
\hline & IRAS $12112+0305$ & 121346.0 & +024838 & 0.0730 & 335 & 12.38 & 3.04 & 23.8 & \\
\hline & IRAS $12540+5708$ & 125614.2 & +565225 & 0.0422 & 188 & 12.42 & 2.94 & 309.9 & Mrk 231 \\
\hline & IRAS $13218+0552$ & 132419.9 & +053705 & 0.2051 & 1011 & 12.44 & 3.50 & 5.3 & \\
\hline & IRAS $13428+5608$ & 134442.1 & +555313 & 0.0378 & 167 & 12.18 & 2.61 & 145.4 & Mrk 273 \\
\hline & IRAS $13451+1232$ & 134733.3 & +121724 & 0.1220 & 571 & 12.21 & 2.46 & 5398.0 & $4 C+12.50$ \\
\hline & IRAS $14059+2000$ & 140818.7 & +194623 & 0.1237 & 580 & 11.94 & 3.40 & 7.5 & \\
\hline & IRAS $14070+0525$ & 140931.2 & +051132 & 0.2644 & 1346 & 12.87 & 4.50 & 5.2 & \\
\hline & IRAS $14553+1245$ & 145743.4 & +123316 & 0.1249 & 585 & 11.87 & 2.33 & 3.8 & \\
\hline & IRAS $15327+2340$ & 153457.1 & +233011 & 0.0181 & 80 & 12.22 & 2.65 & 326.8 & Arp 220 \\
\hline & IRAS 16090-0139 & 161140.5 & -014706 & 0.1339 & 628 & 12.57 & 3.52 & 20.9 & \\
\hline & IRAS $16255+2801$ & 162738.1 & +275452 & 0.1340 & 627 & 11.94 & 2.62 & 5.0 & \\
\hline & IRAS $16300+1558$ & 163221.4 & +155145 & 0.2417 & 1212 & 12.80 & 2.91 & 7.9 & \\
\hline & IRAS $17207-0014$ & 172321.9 & -001701 & 0.0428 & 188 & 12.45 & 3.10 & 82.4 & \\
\hline & IRAS $18368+3549$ & 183835.4 & +355220 & 0.1162 & 536 & 12.24 & 2.91 & 21.0 & \\
\hline & IRAS $18588+3517$ & 190041.2 & +352127 & 0.1067 & 489 & 11.92 & 2.58 & 5.9 & \\
\hline & IRAS 20100-4156 & 201329.5 & -414735 & 0.1296 & 603 & 12.68 & 4.13 & $<5.0$ & \\
\hline & IRAS $20286+1846$ & 203055.5 & +185646 & 0.1347 & 633 & 12.06 & 3.47 & 5.0 & \\
\hline & IRAS $21077+3358$ & 210949.0 & +341020 & 0.1764 & 846 & $12.10-12.24$ & 3.32 & 9.4 & \\
\hline & IRAS $21272+2514$ & 212929.4 & +252750 & 0.1508 & 709 & $11.99-12.14$ & 3.71 & 4.4 & \\
\hline & IRAS $22055+3024$ & 220749.7 & +30 3940 & 0.1269 & 587 & 12.19 & 2.79 & 6.4 & \\
\hline & IRAS $22116+0437$ & 221409.9 & +045224 & 0.1939 & 937 & $12.12-12.32$ & 2.83 & 8.4 & \\
\hline & IRAS $22491-1808$ & 225149.2 & -175223 & 0.0778 & 346 & 12.19 & 2.46 & 5.9 & \\
\hline & IRAS $23028+0725$ & 230520.4 & +074144 & 0.1496 & 701 & $11.86-12.06$ & 3.34 & 19.5 & \\
\hline & IRAS 23233+0946 & 232556.2 & +100249 & 0.1279 & 591 & 12.18 & 2.80 & 11.6 & \\
\hline & IRAS $23365+3604$ & 233901.3 & +362109 & 0.0645 & 283 & 12.19 & 2.52 & 28.7 & \\
\hline \multirow[t]{11}{*}{ Non-masing } & IRAS 00164-1039 & 001850.4 & -102208 & 0.0272 & 113 & 11.36 & $<1.25$ & $<5.0$ & Arp 256 \\
\hline & IRAS $01572+0009$ & 015950.2 & +002341 & 0.1630 & 774 & 12.47 & $<2.12$ & 26.7 & Mrk 1014 \\
\hline & IRAS $05083+7936$ & 051646.4 & +794013 & 0.0537 & 237 & 11.93 & $<1.94$ & 41.4 & \\
\hline & IRAS $06538+4628$ & 065734.4 & +462411 & 0.0214 & 93.6 & 11.24 & $<0.89$ & 64.3 & UGC 3608 \\
\hline & IRAS $08559+1053$ & 085841.8 & +104122 & 0.1480 & 705 & 12.18 & $<1.72$ & $<5.0$ & \\
\hline & IRAS 09437+0317 & 094620.6 & +030330 & 0.0205 & 93.5 & 11.15 & $<1.01$ & $<5.0$ & \\
\hline & IRAS $10565+2448$ & 105918.1 & +243234 & 0.0431 & 194 & 12.04 & $<1.66$ & 57.0 & \\
\hline & IRAS 11119+3257 & 111438.9 & +324133 & 0.1890 & 923 & 12.48 & $<2.07$ & 110.4 & \\
\hline & IRAS $13349+2438$ & 133718.7 & +242303 & 0.1076 & 500 & 11.39 & $<1.72$ & 20.0 & \\
\hline & IRAS $15001+1433$ & 150231.9 & +142135 & 0.1627 & 781 & 12.42 & $<2.04$ & 16.9 & \\
\hline & IRAS $15206+3342$ & 152238.0 & +333136 & 0.1244 & 582 & 12.13 & $<1.75$ & 11.2 & \\
\hline
\end{tabular}


Table 1

(Continued)

\begin{tabular}{|c|c|c|c|c|c|c|c|c|}
\hline IRAS FSC & $\begin{array}{c}\text { R.A. } \\
\text { J2000.0 }\end{array}$ & $\begin{array}{c}\text { Decl. } \\
\text { J2000.0 }\end{array}$ & $z_{\odot}^{\mathrm{a}}$ & $\begin{array}{c}D_{L} \\
\left(h_{70}^{-1} \mathrm{Mpc}\right)\end{array}$ & $\begin{array}{c}\log L_{\mathrm{FIR}}{ }^{\mathrm{b}} \\
\left(h_{70}^{-2} L_{\odot}\right)\end{array}$ & $\begin{array}{l}\log L_{\mathrm{OH}}{ }^{\mathrm{c}} \\
\left(h_{70}^{-2} L_{\odot}\right)\end{array}$ & $\begin{array}{c}f_{1.4 \mathrm{GHz}}^{\mathrm{d}} \\
(\mathrm{mJy})\end{array}$ & Alt. Desig. \\
\hline IRAS $20460+1925$ & 204817.3 & +193654 & 0.1807 & 868 & 12.03 & $<2.15$ & 18.9 & \\
\hline IRAS $23007+0836$ & 230315.6 & +085226 & 0.0163 & 64.9 & 11.43 & $<0.63$ & 181.0 & NGC 7469 \\
\hline IRAS 23394-0353 & 234200.8 & -033655 & 0.0232 & 95.4 & 11.11 & $<1.18$ & $<5.0$ & Arp 295B \\
\hline IRAS $23498+2423$ & 235226.0 & +244017 & 0.2120 & 1037 & 12.44 & $<2.25$ & 6.8 & \\
\hline
\end{tabular}

Notes.

${ }^{a}$ Heliocentric optical redshift (Darling \& Giovanelli 2002a).

${ }^{\mathrm{b}}$ Computed according to the prescription of Sanders $\&$ Mirabel (1996), with a scale factor of $C=1.6$. IRAS photometry is from Sanders et al. (2003); a range

in $L_{\text {FIR }}$ means that the object was not detected by IRAS at $100 \mu \mathrm{m}$.

${ }^{c} \mathrm{OH}$ fluxes are from Darling \& Giovanelli (2002a, 2002b); limits are computed according to Equation (1).

${ }^{\mathrm{d}}$ Flux densities at $1.4 \mathrm{GHz}$ are from the NRAO VLA Sky Survey (Condon et al. 1998).

enough to be resolved, we chose staring mode observations centered on the IR-dominant nuclear region of the galaxy. The only available observations of the double nucleus in IRAS 10485-1447 were centered on the western nucleus. Details of the IRS observations are given in Table 2.

\section{DATA REDUCTION}

\subsection{Dedicated Observations of OHM Galaxies}

The data were processed using the Spitzer Science Center S17.0 data pipeline. We used basic calibrated data (BCD) products for our analysis, having already been corrected for flat fielding, stray light contributions, nonlinear responsivity in the pixels, and "drooping" (an increase in detector pixel voltage that occurs during non-destructive readouts). The two-dimensional BCD images were first medianed over the data cycles at each nod position to remove transient effects such as cosmic rays. For the SL and LL modules, we subtracted the sky contribution by differencing the BCD images for each nod position with the adjacent position in the same module.

The slit sizes of the $\mathrm{SH}$ and $\mathrm{LH}$ modules are too small to permit extraction of a sky background during the same observation. The continuum levels in the HR modules, however, contain strong contributions from scattered zodiacal light. Estimations of the flux at $15 \mu \mathrm{m}$ using SPOT predict zodi contributions within the Spitzer beam ranging from 20 to $80 \mathrm{mJy}$, which in many cases is of comparable magnitude to the expected signal from the galaxies themselves. The wavelength-dependent brightness of the sky contribution means that it cannot be corrected using a simple scaling, and so we did not attempt to further calibrate either HR module. The calibrated LR spectra were thus used for absolute fluxes and the HR spectra for line ratio diagnostics (which are unaffected by continuum levels).

To obtain more accurate measurements of faint lines at longer wavelengths, we took dedicated off-source sky observations in the LH module for all 24 OHMs in our program. Subtraction of the wavelength-dependent background, however, significantly affected the measured line fluxes. For galaxies with background subtraction in only the LH module, this changes the line ratios measured between different HR modules (e.g., [S III] $\lambda 33$ / [S IV] 10.5). We reduced the data both with and without subtraction of the sky backgrounds; the background-subtracted LH spectra are attached as an Appendix.

Following the initial cleaning of the two-dimensional BCD products, we eliminated rogue pixels using the IDL package
IRSCLEAN_MASK ${ }^{7}$. We used rogue pixel masks provided by the SSC for each IRS campaign, and supplemented the standard masks with manual cleaning of each nod and module. The onedimensional spectra were then extracted using the Spitzer IRS Custom Extractor version 2.0. For all modules we used the optimal extraction routine with the standard aperture to improve the $\mathrm{S} / \mathrm{N}$ ratio in faint galaxies.

The LR modules were stitched together to match continuum levels by using a multiplicative scaling. We fixed the LL1 module and then scaled LL2 to LL1, SL1 to LL2, and SL2 to SL1. The mean scaling factors were $1.03 \pm 0.08$ for LL2 to LL1, $1.49 \pm 0.69$ for SL1 to LL2, and $1.37 \pm 0.78$ for SL2 to SL1. We then calibrated the entire LR spectra as a single unit by scaling to the IRS $22 \mu \mathrm{m}$ SUR peakups. The required scaling in the majority of cases was quite small, indicating that the sky subtraction and spectral extraction techniques are robust; the mean scaling factor was $0.94 \pm 0.08$. The accuracy of the overall continuum flux calibration is $\sim 5 \%$ (Houck et al. 2004).

Noisy areas on both ends of the SH and LH orders were trimmed from the one-dimensional spectra. These areas typically encompass a range of 10-30 pixels on the edges of the orders and correspond to areas of decreased sensitivity on the detector. We deliberately trimmed only pixels with an overlapping wavelength range in adjacent orders so that a maximum amount of information is preserved. In isolated cases, we also removed obvious rogue pixels by hand from spectra in which exceptional one-channel features appear in only a single nod.

We calculated a simple figure of merit to measure the $\mathrm{S} / \mathrm{N}$ in the LR data. The data near $\lambda_{\text {rest }}=21 \mu \mathrm{m}$ (a feature-free area near the center of most spectra) are fit with a low-order polynomial; the median flux in that region is then divided by the rms noise to yield the $\mathrm{S} / \mathrm{N}$ (Table 3 ). We note that this parameter is a function of wavelength, as well as the performance and integration time in each spectral module; this is intended to give only a rough estimate for each object. The $\mathrm{S} / \mathrm{N}$ for the samples ranges from $\sim 10$ to 110 , with a median of 35 .

\subsection{Archival Data}

Since the archival OHM and non-masing galaxies did not come from a unified observing program, the version of the Spitzer data pipeline and the level of processing varied slightly from object to object—we used the most recent versions available in the archive (version 15.3.0 or later). The reduction process was identical to that for the OHM galaxies in our program,

\footnotetext{
7 IRSCLEAN_MASK is available at http://ssc.spitzer.caltech.edu/ dataanalysistools/tools/irsclean/
} 
Table 2

IRS Observation Log

\begin{tabular}{|c|c|c|c|c|c|c|c|c|c|}
\hline Object & Date & Peakup Type & SL1 & SL2 & LL1 & LL2 & $\mathrm{SH}$ & $\mathrm{LH}$ & Program \\
\hline IRAS 01355-1814 & & BPU-offset & $60 \times 2$ & $60 \times 2$ & $30 \times 2$ & $30 \times 2$ & $120 \times 4$ & $240 \times 5$ & 4,6 \\
\hline IRAS 01418+1651 & & BPU & $14 \times 2$ & $14 \times 2$ & $14 \times 4$ & $14 \times 4$ & $120 \times 5$ & $60 \times 7$ & 2,3 \\
\hline IRAS $01562+2528$ & 2007 Sep 9 & BPU-offset & $14 \times 7$ & $14 \times 7$ & $30 \times 3$ & $30 \times 3$ & $120 \times 2$ & $60 \times 2$ & 1 \\
\hline IRAS 02524+2046 & 2007 Sep 9 & BPU & $14 \times 7$ & $14 \times 7$ & $30 \times 3$ & $30 \times 3$ & $120 \times 2$ & $60 \times 2$ & 1 \\
\hline IRAS $03521+0028$ & & BPU-offset & $60 \times 2$ & $60 \times 2$ & $30 \times 3$ & $30 \times 3$ & $120 \times 3$ & $60 \times 4$ & 4 \\
\hline IRAS $04121+0223$ & 2007 Oct 5 & BPU-offset & $60 \times 2$ & $60 \times 2$ & $30 \times 3$ & $30 \times 3$ & $120 \times 2$ & $60 \times 2$ & 1 \\
\hline IRAS 04454-4838 & & BPU & $60 \times 2$ & $60 \times 2$ & $30 \times 3$ & $30 \times 3$ & $120 \times 3$ & $240 \times 2$ & 3 \\
\hline IRAS 06487+2208 & 2007 May 4 & BPU-offset & $14 \times 7$ & $14 \times 7$ & $30 \times 3$ & $30 \times 3$ & $120 \times 2$ & $60 \times 2$ & 1 \\
\hline IRAS 07163+0817 & 2007 May 3 & BPU & $14 \times 6$ & $14 \times 6$ & $30 \times 3$ & $30 \times 3$ & $120 \times 2$ & $60 \times 2$ & 1 \\
\hline IRAS $07572+0533$ & 2007 May 4 & BPU & $14 \times 6$ & $14 \times 6$ & $30 \times 3$ & $30 \times 3$ & $120 \times 2$ & $60 \times 2$ & 1 \\
\hline IRAS 08201+2801 & 2007 May 3 & BPU-offset & $14 \times 7$ & $14 \times 7$ & $30 \times 3$ & $30 \times 3$ & $120 \times 2$ & $60 \times 2$ & 1 \\
\hline IRAS 08449+2332 & 2007 May 4 & BPU-offset & $14 \times 6$ & $14 \times 6$ & $30 \times 3$ & $30 \times 3$ & $120 \times 2$ & $60 \times 2$ & 1 \\
\hline IRAS 08474+1813 & 2007 Dec 5 & BPU & $14 \times 6$ & $14 \times 6$ & $30 \times 3$ & $30 \times 3$ & $120 \times 2$ & $60 \times 2$ & 1 \\
\hline IRAS 09039+0503 & & BPU & $60 \times 2$ & $60 \times 2$ & $30 \times 4$ & $30 \times 4$ & $120 \times 4$ & $240 \times 3$ & 5,6 \\
\hline IRAS 09539+0857 & & BPU & $60 \times 2$ & $60 \times 7$ & $30 \times 4$ & $30 \times 4$ & $120 \times 3$ & $240 \times 3$ & 5,6 \\
\hline IRAS $10035+2740$ & 2007 Jun 9 & BPU-offset & $14 \times 7$ & $14 \times 7$ & $30 \times 3$ & $30 \times 3$ & $120 \times 2$ & $60 \times 2$ & 1 \\
\hline IRAS 10039-3338 & & BPU & $14 \times 6$ & $14 \times 6$ & $14 \times 4$ & $14 \times 4$ & $30 \times 6$ & $60 \times 2$ & 3 \\
\hline IRAS $10173+0828$ & & none & $60 \times 1$ & $60 \times 1$ & $14 \times 4$ & $14 \times 4$ & $120 \times 4$ & $60 \times 12$ & $3,7,8$ \\
\hline IRAS 10339+1548 & 2007 Jun 8 & BPU-offset & $14 \times 7$ & $14 \times 7$ & $30 \times 3$ & $30 \times 3$ & $120 \times 2$ & $60 \times 2$ & 1 \\
\hline IRAS 10378+1109 & & BPU-offset & $60 \times 2$ & $60 \times 2$ & $30 \times 3$ & $30 \times 3$ & $120 \times 3$ & $60 \times 4$ & 4 \\
\hline IRAS $10485-1447$ & & BPU & $60 \times 2$ & $60 \times 2$ & $30 \times 4$ & $30 \times 4$ & $120 \times 2$ & $240 \times 2$ & 5,6 \\
\hline IRAS $11028+3130$ & 2007 Jun 9 & BPU-offset & $60 \times 2$ & $60 \times 2$ & $30 \times 3$ & $30 \times 3$ & $120 \times 2$ & $60 \times 2$ & 1 \\
\hline IRAS $11180+1623$ & 2007 Jun 8 & BPU-offset & $14 \times 7$ & $14 \times 7$ & $30 \times 3$ & $30 \times 3$ & $120 \times 2$ & $60 \times 2$ & 1 \\
\hline IRAS 11524+1058 & 2007 Jun 12 & BPU-offset & $14 \times 7$ & $14 \times 7$ & $30 \times 3$ & $30 \times 3$ & $120 \times 2$ & $60 \times 2$ & 1 \\
\hline IRAS 12018+1941 & & BPU-offset & $60 \times 1$ & $60 \times 1$ & $30 \times 3$ & $30 \times 3$ & $120 \times 3$ & $60 \times 4$ & 4 \\
\hline IRAS 12032+1707 & & BPU-offset & $60 \times 2$ & $60 \times 2$ & $30 \times 2$ & $30 \times 2$ & $120 \times 3$ & $240 \times 3$ & 4,6 \\
\hline IRAS $12112+0305$ & & BPU-offset & $14 \times 3$ & $14 \times 3$ & $30 \times 2$ & $30 \times 2$ & $120 \times 2$ & $60 \times 4$ & 4 \\
\hline IRAS $12540+5708$ & & BPU & $14 \times 2$ & $14 \times 2$ & $6 \times 5$ & $6 \times 5$ & $30 \times 6$ & $60 \times 4$ & 9 \\
\hline IRAS 13218+0552 & & BPU-offset & $60 \times 1$ & $60 \times 1$ & $30 \times 3$ & $30 \times 3$ & $120 \times 3$ & $60 \times 4$ & 4 \\
\hline IRAS $13428+5608$ & & BPU & $14 \times 2$ & $14 \times 2$ & $14 \times 2$ & $14 \times 2$ & $30 \times 6$ & $60 \times 4$ & 4 \\
\hline IRAS $13451+1232$ & & $\mathrm{BPU}$ & $14 \times 3$ & $14 \times 3$ & $30 \times 2$ & $30 \times 2$ & $30 \times 6$ & $60 \times 4$ & 4 \\
\hline IRAS 14059+2000 & $2007 \mathrm{Jul} 31$ & BPU-offset & $60 \times 2$ & $60 \times 2$ & $30 \times 3$ & $30 \times 3$ & $120 \times 2$ & $60 \times 2$ & 1 \\
\hline IRAS $14070+0525$ & & BPU-offset & $60 \times 2$ & $60 \times 2$ & $30 \times 2$ & $30 \times 2$ & $120 \times 3$ & $240 \times 2$ & 4 \\
\hline IRAS $14553+1245$ & 2007 Jul 31 & BPU & $60 \times 2$ & $60 \times 2$ & $30 \times 3$ & $30 \times 3$ & $120 \times 2$ & $60 \times 2$ & 1 \\
\hline IRAS $15327+2340$ & & BPU & $14 \times 3$ & $14 \times 3$ & $6 \times 5$ & $6 \times 5$ & $30 \times 6$ & $60 \times 4$ & 10 \\
\hline IRAS 16090-0139 & & BPU-offset & $60 \times 1$ & $60 \times 1$ & $30 \times 3$ & $30 \times 3$ & $120 \times 2$ & $60 \times 4$ & 4 \\
\hline IRAS 16255+2801 & 2006 Sep 17 & $\mathrm{BPU}$ & $60 \times 2$ & $60 \times 2$ & $30 \times 3$ & $30 \times 3$ & $120 \times 2$ & $60 \times 2$ & 1 \\
\hline IRAS $16300+1558$ & & BPU-offset & $60 \times 2$ & $60 \times 2$ & $30 \times 5$ & $30 \times 5$ & $120 \times 4$ & $240 \times 4$ & 4,6 \\
\hline IRAS 17207-0014 & & BPU & $14 \times 3$ & $14 \times 3$ & $14 \times 3$ & $30 \times 2$ & $30 \times 6$ & $60 \times 4$ & 4 \\
\hline IRAS 18368+3549 & 2007 May 1 & BPU & $60 \times 2$ & $60 \times 2$ & $30 \times 3$ & $30 \times 3$ & $120 \times 2$ & $60 \times 2$ & 1 \\
\hline IRAS 18588+3517 & 2006 Nov 20 & BPU & $60 \times 2$ & $60 \times 2$ & $30 \times 3$ & $30 \times 3$ & $120 \times 2$ & $60 \times 2$ & 1 \\
\hline IRAS 20100-4156 & & BPU-offset & $60 \times 1$ & $60 \times 1$ & $30 \times 2$ & $30 \times 2$ & $120 \times 2$ & $60 \times 4$ & 4 \\
\hline IRAS $20286+1846$ & 2006 Nov 20 & BPU & $60 \times 2$ & $60 \times 2$ & $30 \times 3$ & $30 \times 3$ & $120 \times 2$ & $60 \times 2$ & 1 \\
\hline IRAS $21077+3358$ & 2007 Jun 13 & $\mathrm{BPU}$ & $60 \times 2$ & $60 \times 2$ & $30 \times 3$ & $30 \times 3$ & $120 \times 2$ & $60 \times 2$ & 1 \\
\hline IRAS $21272+2514$ & & BPU-offset & $60 \times 2$ & $60 \times 2$ & $30 \times 3$ & $30 \times 3$ & $120 \times 2$ & $240 \times 1$ & 4,11 \\
\hline IRAS $22055+3024$ & 2007 Jun 27 & BPU & $60 \times 2$ & $60 \times 2$ & $30 \times 3$ & $30 \times 3$ & $120 \times 2$ & $60 \times 2$ & 1 \\
\hline IRAS $22116+0437$ & 2006 Dec 21 & BPU-offset & $60 \times 2$ & $60 \times 2$ & $30 \times 3$ & $30 \times 3$ & $120 \times 2$ & $60 \times 2$ & 1 \\
\hline IRAS $22491-1808$ & & BPU-offset & $60 \times 1$ & $60 \times 1$ & $30 \times 2$ & $30 \times 2$ & $120 \times 2$ & $60 \times 4$ & 4 \\
\hline IRAS $23028+0725$ & 2006 Dec 20 & BPU & $14 \times 7$ & $14 \times 7$ & $30 \times 3$ & $30 \times 3$ & $120 \times 2$ & $60 \times 2$ & 1 \\
\hline IRAS 23233+0946 & & BPU & $60 \times 2$ & $60 \times 2$ & $30 \times 4$ & $30 \times 4$ & $120 \times 5$ & $240 \times 4$ & 5,6 \\
\hline IRAS $23365+3604$ & & $\mathrm{BPU}$ & $14 \times 3$ & $14 \times 3$ & $30 \times 2$ & $30 \times 2$ & $30 \times 6$ & $60 \times 4$ & 4 \\
\hline IRAS 00163-1039 & & BPU & $14 \times 3$ & $14 \times 3$ & $14 \times 2$ & $14 \times 2$ & $30 \times 3$ & $60 \times 2$ & 3 \\
\hline IRAS $01572+0009$ & & $\mathrm{BPU}$ & $14 \times 3$ & $14 \times 3$ & $30 \times 2$ & $30 \times 2$ & $30 \times 6$ & $60 \times 4$ & 4 \\
\hline IRAS $05083+7936$ & & BPU & $14 \times 6$ & $14 \times 6$ & $14 \times 4$ & $14 \times 4$ & $30 \times 6$ & $60 \times 2$ & 3 \\
\hline IRAS 06538+4628 & & BPU & $14 \times 3$ & $14 \times 3$ & $14 \times 2$ & $14 \times 2$ & $30 \times 3$ & $60 \times 2$ & 3 \\
\hline IRAS 08559+1053 & & BPU-offset & $60 \times 2$ & $60 \times 2$ & $30 \times 3$ & $30 \times 3$ & $120 \times 3$ & $60 \times 4$ & 12 \\
\hline IRAS 09437+0317 & & BPU & $60 \times 2$ & $60 \times 2$ & $30 \times 3$ & $30 \times 3$ & $120 \times 3$ & $240 \times 2$ & 3 \\
\hline IRAS $10565+2448$ & & BPU & $14 \times 3$ & $14 \times 3$ & $30 \times 2$ & $30 \times 2$ & $30 \times 6$ & $60 \times 4$ & 4 \\
\hline IRAS 11119+3257 & & BPU & $60 \times 1$ & $60 \times 1$ & $30 \times 3$ & $30 \times 3$ & $120 \times 3$ & $60 \times 4$ & 4 \\
\hline IRAS 13349+2438 & & BPU & $14 \times 5$ & $14 \times 5$ & $14 \times 5$ & $14 \times 5$ & $120 \times 5$ & $60 \times 10$ & 13 \\
\hline IRAS $15001+1433$ & & BPU-offset & $60 \times 2$ & $60 \times 2$ & $30 \times 3$ & $30 \times 3$ & $120 \times 3$ & $60 \times 4$ & 4 \\
\hline IRAS 15206+3342 & & BPU-offset & $60 \times 1$ & $60 \times 1$ & $30 \times 3$ & $30 \times 3$ & $120 \times 3$ & $60 \times 4$ & 4 \\
\hline IRAS 20460+1925 & & BPU & $14 \times 5$ & $14 \times 5$ & $14 \times 5$ & $14 \times 5$ & $120 \times 5$ & $60 \times 10$ & 13 \\
\hline
\end{tabular}


Table 2

(Continued)

\begin{tabular}{|c|c|c|c|c|c|c|c|c|c|}
\hline Object & Date & Peakup Type & SL1 & SL2 & LL1 & LL2 & $\mathrm{SH}$ & LH & Program \\
\hline IRAS $23007+0836$ & & BPU & $14 \times 2$ & $14 \times 2$ & $6 \times 5$ & $6 \times 5$ & $30 \times 4$ & $60 \times 2$ & 14 \\
\hline IRAS $23394-0353$ & & BPU & $30 \times 6$ & $30 \times 6$ & $60 \times 2$ & $60 \times 2$ & $14 \times 6$ & $14 \times 4$ & 3 \\
\hline IRAS $23498+2423$ & & BPU-offset & $60 \times 2$ & $60 \times 2$ & $30 \times 2$ & $30 \times 2$ & $120 \times 3$ & $240 \times 2$ & 4 \\
\hline
\end{tabular}

Notes. Spitzer archival data are from programs: (1) 30407 (PI: J. Darling); (2) 3237 (PI: E. Sturm); (3) 30323 (PI: L. Armus); (4) 105 (PI: J. Houck); (5) 2306 (PI: M. Imanishi); (6) 3187 (PI: S. Veilleux); (7) 3605 (PI: C. Bradford); (8) 20549 (PI: R. Joseph); (9) 1442 (PI: L. Armus); (10) 1444 (PI: L. Armus); (11) 20375 (PI: L. Armus); (12) 666 (PI: J. Houck); (13) 61 (PI: G. Rieke); (14) 14 (PI: J. Houck). Exposure times for all modules are given as seconds per cycle $\times$ number of cycles.

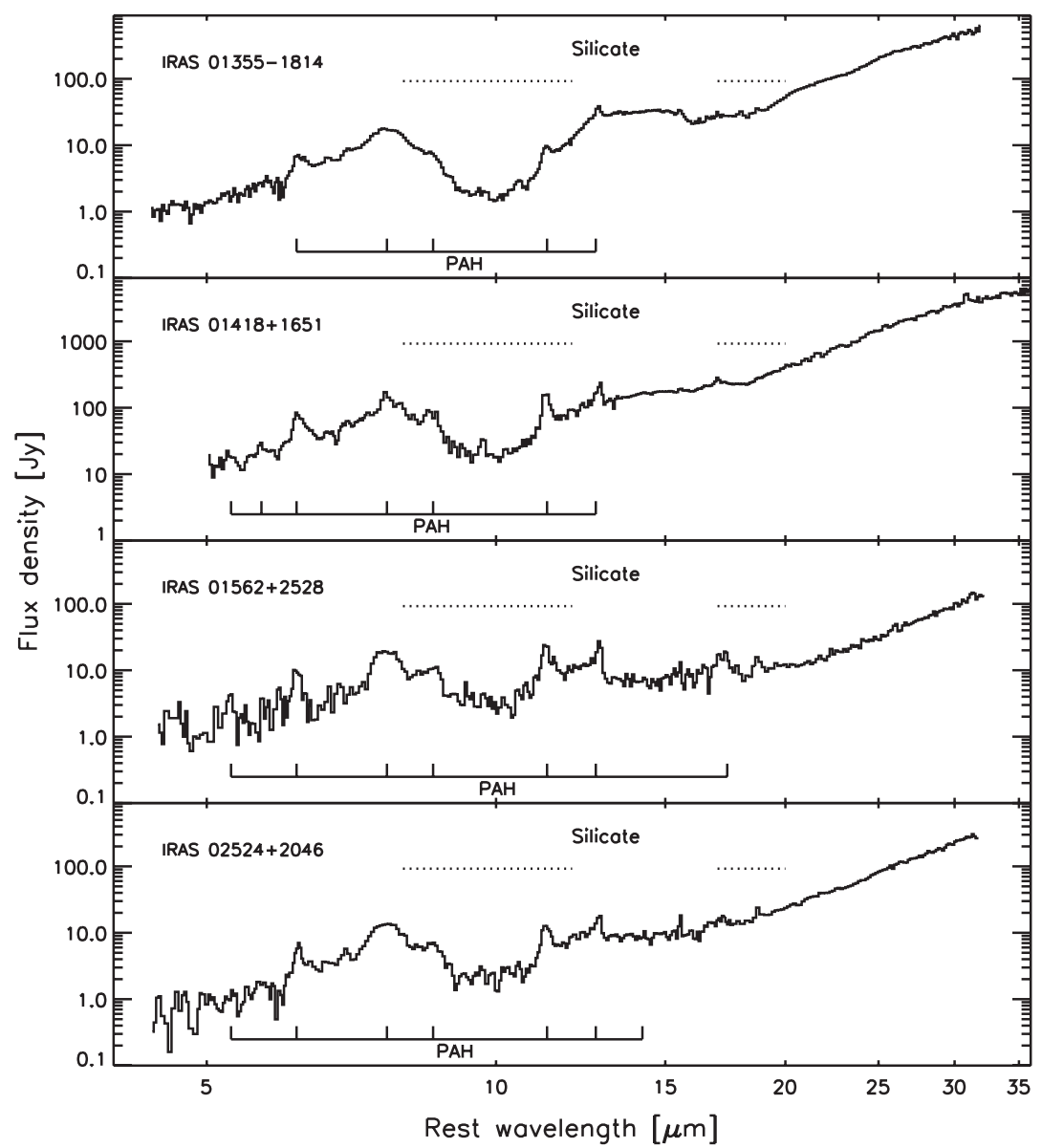

Figure 2. IRS spectra from the low-resolution modules (LR) for OHMs. Spectra for all OHMs and non-masing galaxies are available as an online supplement; portions are shown here for guidance on form and style. All detected PAH emission and absorption features from water ice and silicates are marked.

(An extended version of this figure is available in the online journal.)

with the exception of the LR photometric scaling; since observations in the archive varied in availability of peakup data, we used a variety of sources to calibrate the spectra. In order of priority, we used the IRS dedicated $22 \mu \mathrm{m}$ SUR peakups, IRS acquisition 16 and $22 \mu \mathrm{m}$ DCS peakups, or IRAS $25 \mu \mathrm{m}$ observations (all lying within the coverage of the LL modules). For galaxies from the archive, 17 are calibrated with DCS peakups, 21 with IRAS $25 \mu \mathrm{m}$ photometry, and four are left uncalibrated. The mean scaling factor for the objects without SUR photometry was $1.05 \pm 0.25$, slightly higher than the mean scaling for galaxies in the dedicated OHM program.

Five OHMs and five non-masing galaxies from the archive had both SH and LH sky backgrounds taken simultaneously with the spectroscopic observations; the remainder had no HR sky backgrounds in either module. Since we emphasize uniformity of the observations to the fullest extent possible, all data used for statistical comparisons between the samples use data without HR sky subtraction; line measurements for the backgroundsubtracted objects are given in the Appendix.

The spectra for IRAS 20460+1925 and IRAS 23028+0725 had no flux in the SL modules, most likely due to a pointing error during observations. No SL data from either galaxy are used in our analyses here or in Paper II.

\section{RESULTS}

We show examples of the peakup-scaled LR spectra for the OHMs in Figure 2, with the individual modules stitched together and bonus orders removed. Examples of the HR SH and LH data are shown in Figures 3 and 4; full spectra for all galaxies are available as an online-only extended figures. While individual orders within the HR modules are typically well aligned in flux, 
Table 3

IRS Photometry and Continuum Measurements

\begin{tabular}{|c|c|c|c|c|c|c|}
\hline Object & $\begin{array}{c}16 \mu \text { m Peakup } \\
(\mathrm{mJy})\end{array}$ & $\begin{array}{c}22 \mu \text { m Peakup } \\
(\mathrm{mJy})\end{array}$ & Peakup Type & $\alpha_{15-6}$ & $\alpha_{30-20}$ & $\mathrm{~S} / \mathrm{N}$ \\
\hline IRAS 01355-1814 & $\ldots$ & 45.5 & DCS & $2.8 \pm 0.3$ & $5.2 \pm 0.8$ & 75 \\
\hline IRAS 01418+1651 & $\ldots$ & $\ldots$ & $\ldots$ & $2.2 \pm 0.3$ & $5.2 \pm 0.8$ & 18 \\
\hline IRAS $01562+2528$ & 6.1 & 13.0 & SUR & $1.8 \pm 0.3$ & $5.1 \pm 0.8$ & 11 \\
\hline IRAS 02524+2046 & 10.6 & 21.7 & SUR & $2.0 \pm 0.4$ & $5.7 \pm 1.0$ & 25 \\
\hline IRAS $03521+0028$ & 25.6 & $\ldots$ & DCS & $2.3 \pm 0.4$ & $5.5 \pm 0.8$ & 63 \\
\hline IRAS $04121+0223$ & 10.5 & 22.6 & SUR & $2.6 \pm 0.1$ & $5.9 \pm 0.5$ & 21 \\
\hline IRAS 04454-4838 & $\ldots$ & $\ldots$ & $\ldots$ & $2.7 \pm 0.4$ & $6.1 \pm 0.9$ & 37 \\
\hline IRAS 06487+2208 & 85.0 & 177.5 & SUR & $2.4 \pm 0.2$ & $3.6 \pm 0.8$ & 48 \\
\hline IRAS $07163+0817$ & 12.3 & 28.8 & SUR & $2.5 \pm 0.3$ & $4.8 \pm 1.0$ & 26 \\
\hline IRAS $07572+0533$ & 52.1 & 103.0 & SUR & $2.6 \pm 0.3$ & $3.0 \pm 0.7$ & 55 \\
\hline IRAS $08201+2801$ & 61.8 & 74.3 & SUR & $2.2 \pm 0.2$ & $4.5 \pm 0.7$ & 37 \\
\hline IRAS 08449+2332 & 26.6 & 48.2 & SUR & $2.2 \pm 0.2$ & $4.4 \pm 0.4$ & 52 \\
\hline IRAS $08474+1813$ & 9.5 & 28.7 & SUR & $2.2 \pm 0.5$ & $6.5 \pm 0.6$ & 30 \\
\hline IRAS 09039+0503 & 86.7 & $\ldots$ & DCS & $1.8 \pm 0.5$ & $5.5 \pm 0.7$ & 41 \\
\hline IRAS 09539+0857 & & $\ldots$ & $\ldots$ & $2.2 \pm 0.1$ & $6.0 \pm 0.3$ & 36 \\
\hline IRAS $10035+2740$ & 9.2 & 22.8 & SUR & $2.1 \pm 0.2$ & $6.0 \pm 0.5$ & 23 \\
\hline IRAS $10039-3338$ & $\ldots$ & $\ldots$ & $\ldots$ & $-0.1 \pm 0.1$ & $5.4 \pm 0.3$ & 17 \\
\hline IRAS $10173+0828$ & $\ldots$ & $\ldots$ & $\ldots$ & $2.6 \pm 0.4$ & $6.4 \pm 1.0$ & 48 \\
\hline IRAS $10339+1548$ & 10.7 & 28.1 & SUR & $2.5 \pm 0.3$ & $5.1 \pm 0.7$ & 22 \\
\hline IRAS $10378+1109$ & $\ldots$ & 114.8 & DCS & $2.0 \pm 0.3$ & $4.5 \pm 0.7$ & 35 \\
\hline IRAS $10485-1447$ & $\ldots$ & $\ldots$ & $\ldots$ & $2.5 \pm 0.3$ & $4.8 \pm 0.8$ & 75 \\
\hline IRAS $11028+3130$ & 4.8 & 13.0 & SUR & $2.4 \pm 0.2$ & $7.0 \pm 0.6$ & 26 \\
\hline IRAS $11180+1623$ & 14.8 & 33.6 & SUR & $2.2 \pm 0.3$ & $5.6 \pm 0.9$ & 15 \\
\hline IRAS $11524+1058$ & 8.2 & 14.8 & SUR & $1.5 \pm 0.4$ & $6.1 \pm 0.8$ & 20 \\
\hline IRAS 12018+1941 & 121.7 & $\ldots$ & DCS & $3.0 \pm 0.3$ & $2.9 \pm 0.4$ & 63 \\
\hline IRAS 12032+1707 & $\ldots$ & 73.9 & DCS & $2.2 \pm 0.3$ & $4.2 \pm 0.7$ & 42 \\
\hline IRAS 12112+0305 & 91.9 & $\ldots$ & DCS & $2.3 \pm 0.3$ & $5.3 \pm 0.7$ & 42 \\
\hline IRAS $12540+5708$ & $\ldots$ & $\ldots$ & $\ldots$ & $1.6 \pm 0.3$ & $2.4 \pm 0.7$ & 30 \\
\hline IRAS $13218+0552$ & 212.9 & $\ldots$ & DCS & $0.6 \pm 0.3$ & $2.4 \pm 0.7$ & 100 \\
\hline IRAS $13428+5608$ & $\ldots$ & $\ldots$ & $\ldots$ & $1.9 \pm 1.3$ & $4.6 \pm 0.6$ & 12 \\
\hline IRAS $13451+1232$ & $\ldots$ & $\ldots$ & $\ldots$ & $2.3 \pm 0.4$ & $1.9 \pm 0.7$ & 50 \\
\hline IRAS $14059+2000$ & 12.2 & 26.3 & SUR & $1.1 \pm 0.6$ & $4.9 \pm 0.8$ & 20 \\
\hline IRAS $14070+0525$ & & 30.1 & DCS & $1.6 \pm 0.3$ & $5.6 \pm 0.5$ & 33 \\
\hline IRAS $14553+1245$ & 28.7 & 61.2 & SUR & $2.0 \pm 0.7$ & $4.3 \pm 0.7$ & 22 \\
\hline IRAS $15327+2340$ & $\ldots$ & $\ldots$ & $\ldots$ & $2.4 \pm 0.2$ & $5.5 \pm 0.4$ & 17 \\
\hline IRAS $16090-0139$ & 71.4 & $\ldots$ & DCS & $1.3 \pm 0.4$ & $4.6 \pm 0.6$ & 59 \\
\hline IRAS $16255+2801$ & 16.0 & 36.7 & SUR & $1.4 \pm 0.3$ & $4.7 \pm 0.6$ & 22 \\
\hline IRAS $16300+1558$ & $\ldots$ & 35.0 & DCS & $1.7 \pm 0.5$ & $5.6 \pm 0.9$ & 31 \\
\hline IRAS $17207-0014$ & $\ldots$ & $\ldots$ & $\ldots$ & $1.8 \pm 0.6$ & $6.2 \pm 0.6$ & 28 \\
\hline IRAS $18368+3549$ & 26.6 & 49.8 & SUR & $1.7 \pm 0.5$ & $5.4 \pm 0.8$ & 29 \\
\hline IRAS $18588+3517$ & 43.6 & 92.1 & SUR & $1.5 \pm 0.3$ & $4.8 \pm 0.8$ & 28 \\
\hline IRAS $20100-4156$ & 86.7 & $\ldots$ & DCS & $1.9 \pm 0.2$ & $5.2 \pm 0.7$ & 73 \\
\hline IRAS 20286+1846 & 11.4 & 22.0 & SUR & $2.3 \pm 0.3$ & $5.8 \pm 0.6$ & 20 \\
\hline IRAS $21077+3358$ & 31.1 & 62.1 & SUR & $2.8 \pm 0.3$ & $4.2 \pm 0.6$ & 52 \\
\hline IRAS $21272+2514$ & $\ldots$ & 39.0 & DCS & $2.0 \pm 0.3$ & $5.0 \pm 0.7$ & 36 \\
\hline IRAS $22055+3024$ & 54.5 & 132.4 & SUR & $2.7 \pm 0.3$ & $4.0 \pm 0.7$ & 30 \\
\hline IRAS 22116+0437 & 46.6 & 68.2 & SUR & $2.3 \pm 0.5$ & $4.5 \pm 0.9$ & 35 \\
\hline IRAS 22491-1808 & 87.5 & $\ldots$ & DCS & $2.8 \pm 0.3$ & $5.1 \pm 0.6$ & 28 \\
\hline IRAS $23028+0725$ & 56.4 & 140.7 & SUR & $\ldots$ & $3.2 \pm 0.7$ & 25 \\
\hline IRAS 23233+0946 & $\ldots$ & $\ldots$ & $\ldots$ & $2.1 \pm 0.3$ & $4.7 \pm 0.5$ & 33 \\
\hline IRAS $23365+3604$ & $\ldots$ & $\ldots$ & $\ldots$ & $2.5 \pm 3.1$ & $4.6 \pm 0.5$ & 15 \\
\hline IRAS $00163-1039$ & $\ldots$ & $\ldots$ & $\ldots$ & $2.3 \pm 0.4$ & $2.1 \pm 0.4$ & 34 \\
\hline IRAS 01572+0009 & $\ldots$ & $\ldots$ & $\ldots$ & $1.8 \pm 0.2$ & $2.2 \pm 0.3$ & 81 \\
\hline IRAS $05083+7936$ & $\ldots$ & $\ldots$ & $\ldots$ & $2.0 \pm 0.4$ & $2.8 \pm 0.4$ & 48 \\
\hline IRAS $06538+4628$ & $\ldots$ & $\ldots$ & $\ldots$ & $2.9 \pm 0.3$ & $2.5 \pm 0.4$ & 37 \\
\hline IRAS $08559+1053$ & $\ldots$ & 90.7 & DCS & $1.3 \pm 0.1$ & $2.8 \pm 0.4$ & 64 \\
\hline IRAS 09437+0317 & $\ldots$ & $\ldots$ & $\ldots$ & $1.7 \pm 0.3$ & $2.2 \pm 0.4$ & 19 \\
\hline IRAS $10565+2448$ & $\ldots$ & $\ldots$ & $\ldots$ & $2.1 \pm 0.4$ & $3.2 \pm 0.5$ & 19 \\
\hline IRAS $11119+3257$ & $\ldots$ & $\ldots$ & $\ldots$ & $1.2 \pm 0.1$ & $2.5 \pm 0.3$ & 79 \\
\hline IRAS $13349+2438$ & $\ldots$ & $\ldots$ & $\ldots$ & $0.8 \pm 0.1$ & $0.1 \pm 0.1$ & 110 \\
\hline IRAS $15001+1433$ & $\ldots$ & 135.3 & DCS & $1.9 \pm 0.2$ & $3.4 \pm 0.5$ & 58 \\
\hline IRAS $15206+3342$ & 110.7 & $\ldots$ & DCS & $2.3 \pm 0.4$ & $2.6 \pm 0.4$ & 65 \\
\hline IRAS 20460+1925 & $\ldots$ & $\ldots$ & $\ldots$ & $\ldots$ & $1.1 \pm 0.3$ & 16 \\
\hline IRAS $23007+0836$ & $\ldots$ & $\ldots$ & $\ldots$ & $1.7 \pm 0.1$ & $1.8 \pm 0.4$ & 19 \\
\hline IRAS $23394-0353$ & $\ldots$ & $\ldots$ & $\ldots$ & $1.8 \pm 0.3$ & $2.9 \pm 0.4$ & 37 \\
\hline IRAS $23498+2423$ & 46.5 & $\ldots$ & DCS & $1.0 \pm 0.1$ & $3.1 \pm 0.4$ & 87 \\
\hline
\end{tabular}

Note. Errors in the peakup fluxes are at the $15 \%$ level. 


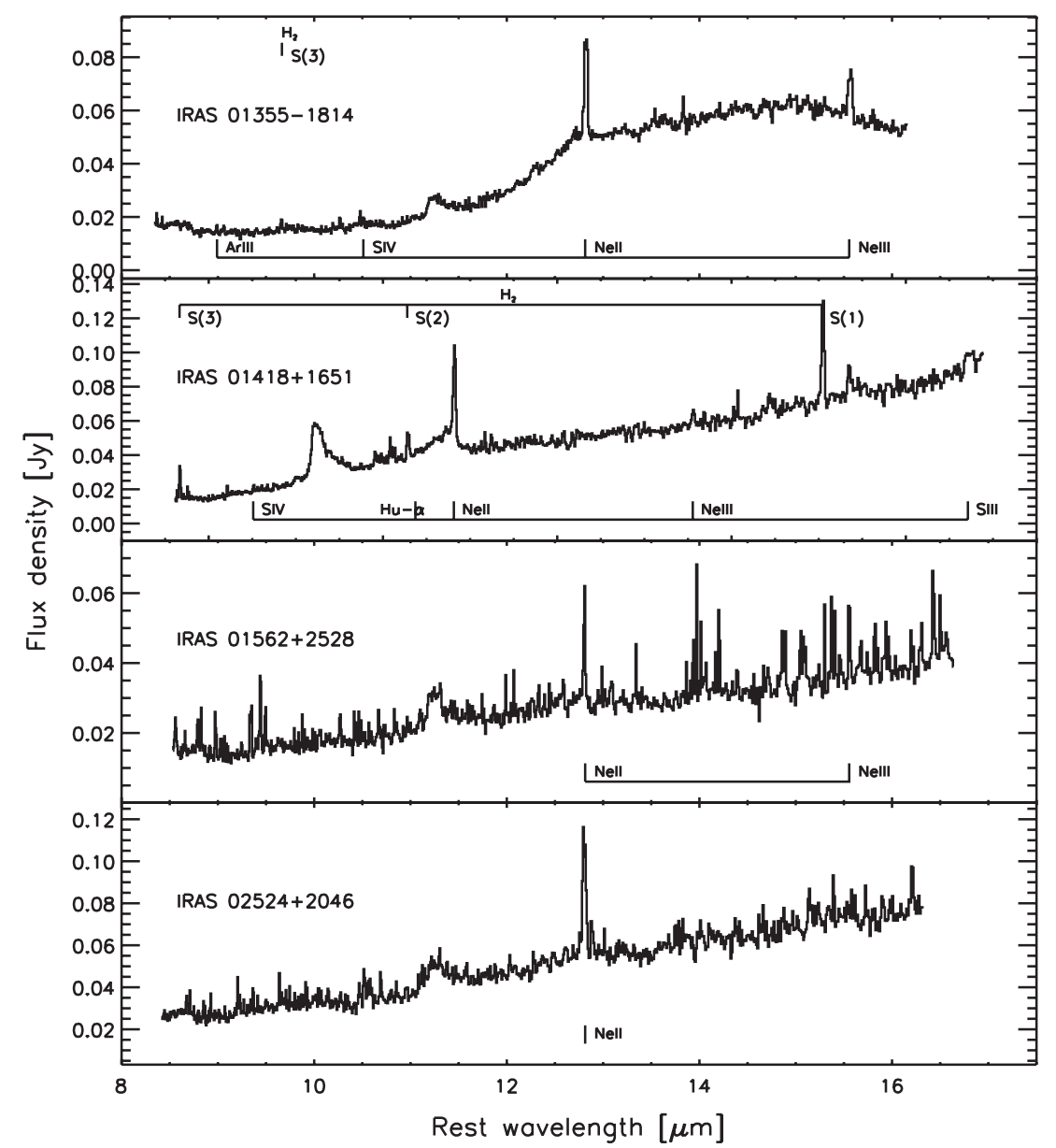

Figure 3. IRS spectra from the short-high module (SH) for OHMs. Spectra for all OHMs and non-masing galaxies are available as an online supplement; portions are shown here for guidance on form and style. All detected atomic and $\mathrm{H}_{2}$ features in each spectra are marked.

(An extended version of this figure is available in the online journal.)

the differences in calibration between the SH and LH modules are clearly apparent when matching the spectra; this is due to a combination of different slit sizes for the SH and LH modules (a factor of $\sim 4$ ) and the lack of separate sky subtraction for the $\mathrm{SH}$ modules. For this reason, as well as emphasizing the narrow atomic and molecular features visible in the HR spectra, we display separate plots for the SH and LH modules.

A small amount of the archival objects have previously published full IRS spectra (Armus et al. 2004; Weedman et al. 2005; Armus et al. 2007), mainly consisting of bright, nearby galaxies. Farrah et al. (2007) published HR spectra for roughly half of our archival OHM hosts. While many papers use the available data from the GTO programs, however, the majority of objects extracted from the archive have no published spectra, although some data are used in larger studies of ULIRG properties (e.g., Higdon et al. 2006; Desai et al. 2007; Hao et al. 2007). The spectra for many of the archival galaxies are thus presented here for the first time.

Comparison of our data with the few spectra of objects previously published (e.g., Mrk 1014, Arp 220, NGC 7469) revealed no significant differences in spectral shape or detection of individual features. Measurements of line flux and equivalent widths (EWs), however, may be affected by the photometric scaling and/or line-fitting routines used; for this reason, we chose to reduce all data in a uniform matter.

For many of our HR spectra, especially those with low $\mathrm{S} / \mathrm{N}$, there exist individual spikes that do not correspond to any identified feature (see IRAS $01562+2528$ for a prominent example). These features are typically 1-2 channels wide, much narrower than the expected line width for an unresolved feature. We regard such features as spurious, possibly caused by hot pixels or other instrumental conditions that are not corrected by our cleaning routines. All features we regard as valid detections are listed in the data tables, with the locations of the most common features marked on the spectra themselves.

\subsection{Continuum}

The continuum emission for all objects in the OHM sample has a relatively homogenous spectral shape over the range of the IRS, although differences in spectral shape between the two samples do appear and are explored in Paper II. Figure 5 shows the individual objects overlaid with a template generated by medianing the flux in each wavelength bin from all galaxies. The template bears a close resemblance to starbursting ULIRG spectra seen in previous surveys (Hao et al. 2007; Weedman \& Houck 2009). The LR spectrum clearly shows silicate absorption at 9.7 and $18 \mu \mathrm{m}$ and water ice absorption at $6 \mu \mathrm{m}$. LR emission features are dominated by the broad polycyclic aromatic hydrocarbon (PAH) features from 6 to $13 \mu \mathrm{m}$, with weaker contributions from neon, sulfur, and molecular hydrogen also visible.

The continuum data from $\lambda_{\text {rest }}=20$ to $30 \mu \mathrm{m}$ are in most cases well characterized by a power-law fit, with the short 


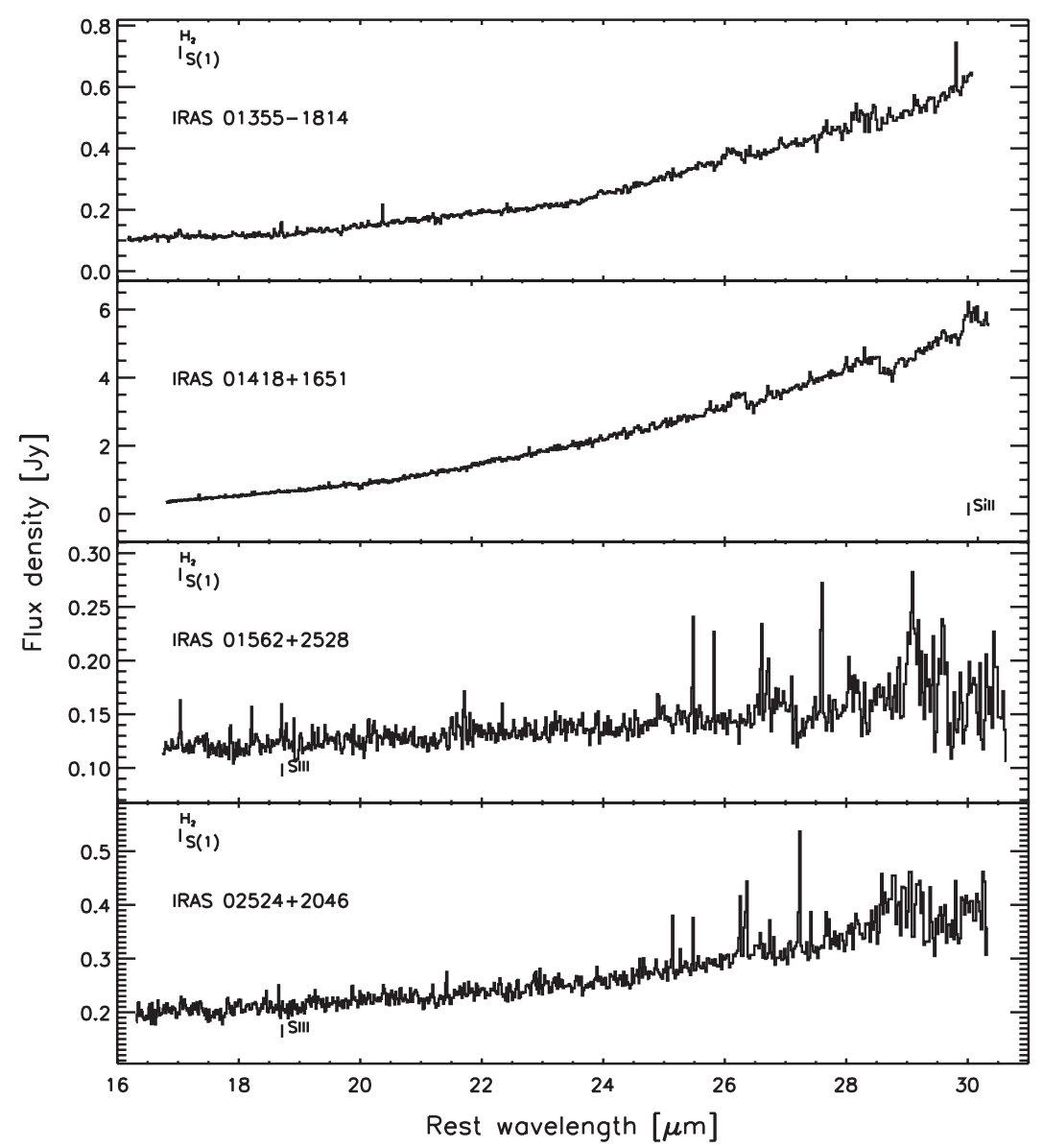

Figure 4. IRS spectra from the long-high module (LH) for OHMs. Spectra for all OHMs and non-masing galaxies are available as an online supplement; portions are shown here for guidance on form and style. All detected atomic and $\mathrm{H}_{2}$ emission features are marked.

(An extended version of this figure is available in the online journal.)

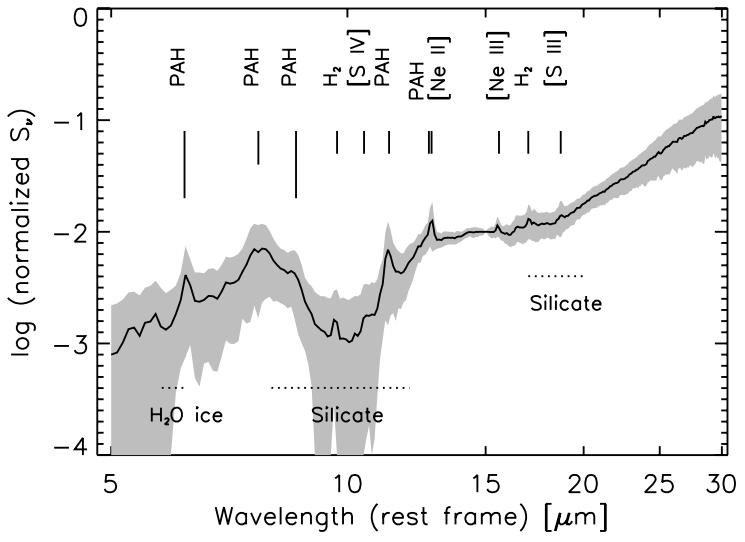

Figure 5. Low-resolution spectrum of all galaxies in our sample normalized at $S_{\lambda}=15 \mu \mathrm{m}$. The black spectrum is the composite template made from an errorweighted median of the individual galaxies; the gray shaded area shows the $1 \sigma$ envelope for each resolution element (exaggerated toward negative values in log space).

wavelength break occurring near the $18 \mu \mathrm{m}$ silicate feature and the long wavelength end cut off by the spectral range of the IRS. Shortward of $15 \mu \mathrm{m}$, the continuum becomes increasingly contaminated by individual absorption and emission features, especially from PAH emission and the deep silicate absorption at $9.7 \mu \mathrm{m}$. Following Brandl et al. (2006), we fit a spectral index to the continuum in two components, with $\alpha_{30-20}$ measuring the relatively feature-free flux from 20 to $30 \mu \mathrm{m}$ and $\alpha_{15-6}$ measur- ing the contribution from 5.3 to $14.8 \mu \mathrm{m}$; the wavelengths are slightly shifted to avoid contamination from water ice at $6 \mu \mathrm{m}$ and [Ne III] at $15.6 \mu \mathrm{m}$ (Table 3).

The mean 15-6 spectral index for the entire OHM sample is $\alpha_{15-6}=2.1 \pm 0.6$; the mean 30-20 slope is $\alpha_{30-20}=4.8 \pm 1.1$. The shallowest 15-6 slope occurs for IRAS 10039-3338 $\left(\alpha_{15-6}=-0.1\right)$, an object with weak PAH features and very strong silicate absorption; the steepest index occurs for IRAS $12018+1941\left(\alpha_{15-6}=3.0\right)$, which has moderate PAH and line emission features and a nearly constant spectral index over the entire mid-IR range. Steeper 15-6 indices are likely due to a combination of smaller relative quantities of warm dust (thermal blackbodies of $\sim 300 \mathrm{~K}$ peaking near $10 \mu \mathrm{m}$ ) and larger quantities of cooler dust.

The shallowest 30-20 slope occurs for IRAS 13451+1232 $\left(\alpha_{30-20}=1.9\right)$; the LR spectrum for this object more closely resembles that seen in Seyfert galaxies and PG quasars (Schweitzer et al. 2006; Hao et al. 2007), with weak PAH emission and shallower silicate absorption. The continuum emission for this object is also much closer to being uniform over the entire range of the IRS; the difference between the two spectral indices is only $\Delta \alpha=-0.4$, compared to an average of $\Delta \alpha=2.7$ for the entire sample. This behavior is more typical of non-thermal emission that can extend over many decades with the same index. The steepest 30-20 emission measured is from IRAS $11028+3130\left(\alpha_{30-20}=7.0\right)$; the galaxy shows moderate $\mathrm{PAH}$ and line emission features, but a flat continuum between 12 and $20 \mu \mathrm{m}$. 
The non-masing galaxies have average spectral indices of $\alpha_{15-6}=1.8 \pm 0.6$ and $\alpha_{30-20}=2.5 \pm 0.9$. A full statistical analysis of the values for the two samples (particularly $\alpha_{30-20}$ ) is presented in Paper II.

\subsection{Atomic Emission Lines}

We measured emission from atomic and molecular lines using the standard packages in the Spectroscopic Modeling Analysis and Reduction Tool (SMART) version 6.2.4 (Higdon et al. 2004). A simple Gaussian is a good fit for virtually all HR lines in the sample; in cases where lines are blended (such as the [Ne v]/ $[\mathrm{Cl} \mathrm{II}]$ and [O IV] $/[\mathrm{Fe}$ II] complexes), we used a multi-Gaussian fit centered at the redshifted rest wavelengths of the expected transitions. To compute upper limits for non-detections, we use the $3 \sigma$ noise measured from the surrounding continuum and a Gaussian shape with an FWHM estimated from detected lines. Accuracy for all measured line fluxes is on the order of $\sim 10 \%$.

The most common lines detected are the forbidden [Ne II] $\lambda 12.814$ and [Ne III] $\lambda 15.555$ transitions (Table 4). [Ne II] is observed in nearly the entire sample, with detections in $50 / 51$ OHMs and 14/15 non-masing galaxies. The only exceptions are the OHM IRAS $11028+3130$ and the non-maser IRAS 20460+1925. [Ne III] is also common, detected in 43/51 OHMs and 14/15 non-masing galaxies. Other common lines are the [S III] $\lambda 18.713$ (detected in $\sim 80 \%$ of galaxies) and [S IV] $\lambda 10.511(\sim 50 \%)$. [Ar III] $\lambda 8.991$ is detected in 15 OHMs and 5 non-masing galaxies, but the redshifted line is not visible in the $\mathrm{SH}$ module for archived objects at $z<0.1$.

We detect "rarer" line transitions that appear in less than $15 \%$ of our sample, including [Ne v] $\lambda 14.322$ and $\lambda 24.318,[\mathrm{Fe}$ II] $\lambda 17.936$ and $\lambda 25.988$, and [O IV] $\lambda 25.890$. IRAS $10339+1548$ is the only galaxy in the sample with detections for all atomic transitions listed above. Eight galaxies also show the nonforbidden H I 7-6 transition (Humphreys- $\alpha$ ) at $12.368 \mu \mathrm{m}$.

[S III] $\lambda 33.481$ and [Si II] $\lambda 34.815$ are commonly observed in ULIRGs for which the redshifted transitions appear within the red edge of the LH module. Farrah et al. (2007) detected [S III] 333.481 in roughly half of all ULIRGs with $z<0.06$, all of which show the stronger [S III] $\lambda 18.713$ associated transition. For our sample, however, many galaxies have the lines redshifted beyond the range of the IRS; when visible, the lines lie at the far red edge of the 11th order in the LH module, an area with high noise and decreased sensitivity with respect to neighboring orders.

For galaxies taken from the Spitzer archive, we compared our HR measurements with those appearing in the sample of Farrah et al. (2007). The agreement between detected lines is good for nearly all objects; however, Farrah et al. (2007) report detections of [Ne v] in IRAS 11119+3257, [O IV] in IRAS 13451+1232, and $\mathrm{H}_{2} S(0)$ and $S(2)$ lines in IRAS $01572+0009$ and IRAS $23498+2423$ which we fail to confirm. Limits for the measured fluxes are given in Tables 4 and 5.

The IRS is designed to make accurate measurements of narrow atomic transitions in the SH and LH modules; however, several lines are also detected in the LR modules, most often the powerful neon, sulfur, and $\mathrm{H}_{2}$ transitions. The only emission lines visible in the LR modules without a corresponding detection in $\mathrm{HR}$ are the $\mathrm{H}_{2} S(7)$ line at $5.5 \mu \mathrm{m}$ and the $\mathrm{H}_{2}$ $S(5) /[$ Ar II] complex near $6.7 \mu \mathrm{m}$; this is due to the SH lowwavelength cutoff at $\lambda_{\text {rest }}=8.2-9.0 \mu \mathrm{m}$, depending on the redshift of the galaxy. All other atomic emission features observed in the LR modules have corresponding detections in HR; furthermore, blending of narrow lines makes accurate measurements of flux difficult in the LR modules. For isolated lines with welldefined surrounding continuum, our fluxes are consistent for measurements in both low and high resolution.

We note the presence of two features which have no obvious identifications occurring in the LH spectra for multiple objects: one is an emission feature seen near $29 \mu \mathrm{m}$ (a prominent example occurs for IRAS $17539+2935$ ) and the second is an absorption feature near $30 \mu \mathrm{m}$. The two are often paired and are seen in $\sim 50 \%$ of the galaxies observed. The rest wavelengths of the transitions, however, vary significantly from object to object (with a standard deviation of $\sigma_{\lambda} \simeq 0.7 \mu \mathrm{m}$ ), while the observed wavelengths are nearly fixed $\left(\sigma_{\lambda} \lesssim 0.05 \mu \mathrm{m}\right)$. This implies that the features are either artifacts of the extraction process or that both the emission and absorption come from unidentified foreground features with little to no Doppler shift. Given that we see no evidence for these unidentified lines in any of the LR spectra (which should be detectable, given the high $\mathrm{S} / \mathrm{N}$ for many of the features), we consider both to be spurious.

\subsection{Molecular Hydrogen}

We detected multiple emission lines from the pure rotational series of molecular hydrogen in both OHMs and non-masing galaxies. At redshifts of $z \lesssim 0.1$, transitions from $\mathrm{H}_{2} S(0)$ at $28.22 \mu \mathrm{m}$ to $\mathrm{H}_{2} S(3)$ at $9.67 \mu \mathrm{m}$ are visible in the HR modules; in addition, the LR module is capable of detecting lines as far out as the $S(7)$ transition at $5.51 \mu \mathrm{m}$. In each case, the line number (e.g., 0 for $\mathrm{H}_{2} S(0)$ ) indicates the rotational quantum number of the lower state $(J=2 \rightarrow 0)$ for the quadrupole $S$-branch transition. $\Delta J=2$ results in two separate branches: ortho (parallel nuclear spin, odd $J$ ) and para (anti-parallel nuclear spin, even $J$ ).

We detected at least one $\mathrm{H}_{2}$ line in 49/51 OHMs and 13/15 non-masing galaxies, with $S(1)$ seen in all objects for which at least one molecular hydrogen transition is reported. The higherorder $S(2)$ and $S(3)$ lines are seen in roughly $2 / 3$ of the sample, while the para ground-state $S(0)$ transition is detected in only $\sim 15 \%$ of the sample. Our line detection rate is consistent with results from the SINGS galaxies examined in Roussel et al. (2007) and the ULIRG sample of Higdon et al. (2006). Lower detection rates of $S(0)$ and $S(2)$ are likely due to a combination of the intrinsic ortho-para ratio as well as rising continuum levels near $28 \mu \mathrm{m}$ that can obscure weak line emission by $S(0)$. Higdon et al. (2006) find that the $S(2) / S(3)$ ratios are consistent with no significant differential extinction for the two lines, which is supported by numerous detections in our sample of $S(3)$ line emission superimposed on optically deep silicate absorption near $9.7 \mu \mathrm{m}$. We thus applied no extinction or reddening corrections to the line fluxes (Table 5).

We did not detect the $S(6)$ line in any galaxy, while $S(4)$ showed a single detection in IRAS $16300+1558$. Since these lines are excited by hotter gas $(T \sim 1000 \mathrm{~K})$, they are typically weaker than the lower states which probe the larger reservoir of cool gas. In addition, both lines are only visible in the LR modules at $z \sim 0.1$. This means that deblending is a significant issue, since both lines lie near broad PAH emission complexes. Eleven OHMs and one non-masing galaxy show the unresolved $S(7)$ ortho line at $5.51 \mu \mathrm{m}$ in the SL module.

Measurement of the $S(5)$ line presents a particular problem due to its location in a crowded section of the spectra. Its rest wavelength of $6.91 \mu \mathrm{m}$ lies near the [Ar II] feature at $6.99 \mu \mathrm{m}$; in addition, both features are bracketed by possible hydrocarbon absorption at 6.85 and $7.25 \mu \mathrm{m}$. This not only creates difficulties in establishing a reliable continuum, but also in deblending the 
Table 4

Atomic Line Fluxes for High-resolution Spectra

\begin{tabular}{|c|c|c|c|c|c|c|c|c|c|c|c|c|c|c|}
\hline $\begin{array}{c}\text { Object } \\
\lambda_{\text {rest }}(\mu \mathrm{m})\end{array}$ & $\begin{array}{c}\text { [Ar III] } \\
8.991\end{array}$ & $\begin{array}{l}\text { [S IV] } \\
10.511\end{array}$ & $\begin{array}{l}\text { HI 7-6 } \\
12.368\end{array}$ & $\begin{array}{l}{[\mathrm{Ne} I \mathrm{II}]} \\
12.814\end{array}$ & $\begin{array}{l}{[\mathrm{Ne} \mathrm{V}]} \\
14.322\end{array}$ & $\begin{array}{l}{[\mathrm{Cl} I \mathrm{II}]} \\
14.369\end{array}$ & $\begin{array}{l}\text { [Ne III] } \\
15.555\end{array}$ & $\begin{array}{l}{[\mathrm{Fe} \mathrm{II}]} \\
17.936\end{array}$ & $\begin{array}{l}\text { [S III] } \\
18.713\end{array}$ & $\begin{array}{l}{[\mathrm{Ne} \mathrm{V}]} \\
24.318\end{array}$ & $\begin{array}{l}\text { [O IV] } \\
25.890\end{array}$ & $\begin{array}{l}{[\mathrm{Fe} \text { II }]} \\
25.988\end{array}$ & $\begin{array}{l}\text { [S III] } \\
33.481\end{array}$ & $\begin{array}{l}{[\mathrm{Si} \text { III }} \\
34.815\end{array}$ \\
\hline IRAS 01355-1814 & 0.15 & 0.10 & $<0.20$ & 2.45 & $<0.27$ & $<0.25$ & 0.89 & $<0.51$ & $<1.52$ & $<0.78$ & $<1.28$ & $<0.82$ & - & - \\
\hline IRAS 01418+1651 & - & 0.15 & 0.13 & 4.17 & $<0.46$ & $<0.29$ & 0.41 & $<0.67$ & 0.94 & $<3.84$ & $<3.52$ & $<4.05$ & $<24.62$ & 19.43 \\
\hline IRAS 01562+2528 & $<0.77$ & $<1.93$ & $<0.30$ & 1.07 & $<0.91$ & $<0.75$ & 0.77 & $<0.77$ & 0.67 & $<0.73$ & $<2.06$ & $<1.29$ & - & - \\
\hline IRAS 02524+2046 & $<0.63$ & $<1.01$ & $<0.36$ & 2.39 & $<0.68$ & $<0.56$ & $<0.97$ & $<1.11$ & 0.47 & $<1.04$ & $<0.96$ & $<0.59$ & - & - \\
\hline IRAS 03521+0028 & $<0.34$ & $<0.40$ & $<0.41$ & 2.72 & $<0.36$ & $<0.24$ & 1.11 & 1.70 & 1.18 & $<1.02$ & $<0.93$ & $<0.59$ & - & - \\
\hline IRAS $04121+0223$ & 0.35 & $<0.84$ & $<0.41$ & 1.81 & $<1.01$ & $<0.99$ & $<0.87$ & $<0.92$ & 0.93 & $<1.06$ & 0.97 & $<1.34$ & - & - \\
\hline IRAS 04454-4838 & - & 0.47 & $<0.74$ & 2.04 & $<0.75$ & $<0.61$ & 0.39 & $<0.27$ & $<1.00$ & $<7.75$ & $<9.59$ & $<6.29$ & 0.90 & $<87.49$ \\
\hline IRAS 06487+2208 & 1.71 & 1.78 & $<0.81$ & 11.75 & $<1.44$ & $<1.19$ & 9.45 & $<0.87$ & 4.89 & $<1.36$ & 0.48 & 1.31 & - & - \\
\hline IRAS 07163+0817 & 0.44 & $<1.05$ & $<0.22$ & 3.19 & $<0.46$ & $<0.39$ & 0.33 & $<1.04$ & 0.81 & $<1.73$ & $<0.78$ & $<0.71$ & - & - \\
\hline IRAS $07572+0533$ & $<1.69$ & $<0.87$ & $<0.40$ & 1.74 & $<0.79$ & $<0.65$ & $<0.89$ & $<0.61$ & $<1.15$ & $<1.37$ & $<1.53$ & $<1.03$ & - & - \\
\hline IRAS 08201+2801 & $<0.70$ & $<2.74$ & $<0.64$ & 2.23 & $<0.60$ & $<0.41$ & 0.88 & $<0.84$ & 0.41 & $<0.50$ & $<0.66$ & $<0.50$ & - & - \\
\hline IRAS 08449+2332 & $<0.94$ & $<0.71$ & $<0.64$ & 3.60 & $<2.03$ & $<1.64$ & 1.57 & $<0.60$ & 1.62 & $<0.73$ & $<0.69$ & $<0.40$ & - & - \\
\hline IRAS 08474+1813 & $<0.76$ & $<0.64$ & $<0.36$ & 1.26 & $<1.10$ & $<0.89$ & $<1.10$ & $<1.09$ & 0.86 & $<1.27$ & $<3.04$ & $<1.86$ & - & - \\
\hline IRAS 09039+0503 & 0.11 & $<0.27$ & $<0.85$ & 3.68 & $<0.46$ & $<0.38$ & 1.17 & $<0.85$ & 0.88 & $<0.55$ & $<1.11$ & 0.48 & - & - \\
\hline IRAS 09539+0857 & $<0.63$ & $<0.43$ & $<0.30$ & 1.25 & $<0.86$ & $<0.69$ & $<1.88$ & $<1.82$ & $<1.02$ & $<1.41$ & $<2.05$ & $<1.36$ & - & - \\
\hline IRAS $10035+2740$ & $<0.89$ & $<0.75$ & $<0.48$ & 1.82 & $<0.42$ & $<0.31$ & 0.57 & $<0.97$ & 0.78 & $<0.95$ & $<2.11$ & $<1.37$ & - & - \\
\hline IRAS 10039-3338 & - & 0.93 & $<1.25$ & 16.69 & $<1.21$ & $<1.10$ & 3.93 & $<0.81$ & 6.57 & $<14.49$ & $<11.09$ & $<7.54$ & $<10.49$ & 101.40 \\
\hline IRAS 10173+0828 & - & $<0.29$ & $<0.34$ & 1.71 & $<0.33$ & $<0.32$ & 0.46 & $<0.42$ & $<1.36$ & $<1.33$ & $<2.42$ & $<1.61$ & 2.55 & 81.70 \\
\hline IRAS 10339+1548 & 1.00 & 0.55 & $<0.34$ & 1.25 & 1.24 & $<1.13$ & 2.03 & 0.49 & 1.17 & 0.52 & 2.85 & 0.89 & - & - \\
\hline IRAS $10378+1109$ & 0.34 & $<0.25$ & $<0.35$ & 3.95 & $<0.61$ & $<0.63$ & 0.68 & $<0.95$ & 1.82 & $<0.87$ & $<1.99$ & $<1.30$ & - & - \\
\hline IRAS $10485-1447$ & $<1.14$ & $<0.20$ & $<0.23$ & 1.99 & $<0.44$ & $<0.48$ & 0.36 & $<0.87$ & $<1.08$ & $<0.91$ & $<1.08$ & $<0.66$ & - & - \\
\hline IRAS $11028+3130$ & $<1.06$ & $<0.70$ & $<0.28$ & $<0.63$ & $<0.44$ & $<0.38$ & $<0.74$ & $<0.47$ & $<0.71$ & $<3.07$ & $<4.45$ & $<3.14$ & - & - \\
\hline IRAS $11180+1623$ & $<0.80$ & $<0.70$ & $<0.36$ & 1.98 & $<0.50$ & $<0.39$ & 0.62 & $<0.71$ & 0.89 & $<0.85$ & $<1.00$ & $<0.69$ & - & - \\
\hline IRAS $11524+1058$ & $<0.78$ & $<0.56$ & $<0.35$ & 7.82 & $<0.43$ & $<0.37$ & $<0.77$ & $<0.52$ & $<1.61$ & $<1.49$ & $<0.93$ & $<0.70$ & - & - \\
\hline IRAS 12018+1941 & $<0.27$ & $<0.28$ & $<0.59$ & 2.73 & $<0.52$ & $<0.38$ & 0.64 & $<1.01$ & $<1.32$ & $<1.22$ & $<1.71$ & $<1.30$ & - & - \\
\hline IRAS 12032+1707 & $<1.33$ & 0.13 & $<0.48$ & 4.98 & $1.39:$ & $<1.51$ & 1.57 & $<1.22$ & 1.18 & 0.66 & $<1.50$ & $<1.00$ & - & - \\
\hline IRAS 12112+0305 & - & 0.43 & $<0.88$ & 13.06 & $<0.76$ & $<0.55$ & 3.37 & 0.68 & 4.32 & $<1.50$ & $<7.56$ & $<4.65$ & 8.77 & - \\
\hline IRAS $12540+5708$ & - & $<11.45$ & $<4.40$ & 17.95 & $<8.05$ & $<6.86$ & 9.36 & $<4.40$ & $<5.05$ & $<22.99$ & $<18.84$ & $<14.43$ & $<49.65$ & $<163.66$ \\
\hline IRAS 13218+0552 & $<0.66$ & $<0.54$ & $<0.46$ & 0.84 & $<0.71$ & $<1.23$ & $<1.24$ & $<0.78$ & $<2.27$ & $<1.85$ & $<2.93$ & $<1.76$ & - & - \\
\hline IRAS 13428+5608 & - & 7.69 & $<2.42$ & 41.21 & 10.62 & $<5.37$ & 29.05 & $<1.32$ & 16.67 & 9.28 & 75.13 & $<29.79$ & 28.14 & $<134.61$ \\
\hline IRAS $13451+1232$ & 0.57 & 1.57 & $<0.70$ & 4.71 & 0.71 & $<0.88$ & 4.73 & $<1.19$ & 1.15 & $<2.50$ & $<5.17$ & $<1.85$ & - & - \\
\hline IRAS 14059+2000 & $<0.64$ & 0.35 & $<0.53$ & 2.83 & $<0.88$ & $<1.00$ & 2.65 & $<0.97$ & 1.68 & $<1.04$ & $<1.70$ & $<1.11$ & - & - \\
\hline IRAS 14070+0525 & $<0.19$ & $<0.18$ & $<0.27$ & 1.33 & $<0.42$ & $<0.33$ & 2.01 & $<0.53$ & $<0.77$ & $<1.25$ & $<1.98$ & $<1.37$ & - & - \\
\hline IRAS 14553+1245 & 0.47 & 0.71 & $<0.25$ & 3.24 & $<1.55$ & $<1.29$ & 3.10 & $<0.89$ & 1.58 & $<0.89$ & $<2.38$ & $<1.49$ & - & - \\
\hline IRAS $15327+2340$ & - & $<0.79$ & $<3.60$ & 61.13 & $<6.29$ & $<5.29$ & 6.89 & $<1.04$ & 5.19 & $<24.96$ & $<24.33$ & $<15.22$ & $<151.68$ & $<202.18$ \\
\hline
\end{tabular}




\begin{tabular}{|c|c|c|c|c|c|c|c|c|c|c|c|c|c|c|}
\hline Object & [Ar III] & [S IV] & HI 7-6 & {$[\mathrm{Ne} \mathrm{II}]$} & {$[\mathrm{Ne} \mathrm{V}]$} & {$[\mathrm{Cl} \mathrm{II}]$} & [Ne III] & {$[\mathrm{Fe} \mathrm{II}]$} & [S III] & {$[\mathrm{Ne} \mathrm{V}]$} & [O IV] & {$[\mathrm{Fe} \mathrm{II}]$} & [S III] & [Si II] \\
\hline$\lambda_{\text {rest }}(\mu \mathrm{m})$ & 8.991 & 10.511 & 12.368 & 12.814 & 14.322 & 14.369 & 15.555 & 17.936 & 18.713 & 24.318 & 25.890 & 25.988 & 33.481 & 34.815 \\
\hline IRAS 16090-0139 & 0.49 & 0.10 & $<0.49$ & 6.72 & $<0.65$ & $<0.76$ & 2.26 & 1.03 & 3.07 & $<3.95$ & 1.12 & $<2.23$ & - & - \\
\hline IRAS $16255+2801$ & 0.18 & 0.20 & $<0.27$ & 1.73 & $<0.82$ & $<0.68$ & 1.18 & $<5.36$ & 1.54 & $<12.06$ & $<7.16$ & $<4.83$ & - & - \\
\hline IRAS $16300+1558$ & 0.13 & $<0.15$ & $<0.28$ & 2.27 & $<0.33$ & $<0.25$ & 0.42 & $<1.20$ & 0.50 & $<1.23$ & $<0.88$ & $<0.70$ & - & - \\
\hline IRAS 17207-0014 & - & 0.40 & $<2.12$ & 38.84 & $<0.93$ & $<0.69$ & 8.42 & $<0.51$ & 6.39 & $<6.40$ & $<11.27$ & $<7.70$ & 12.24 & 46.91 \\
\hline IRAS $18368+3549$ & $<0.42$ & $<0.81$ & 0.11 & 6.91 & $<0.34$ & $<0.43$ & 1.07 & $<0.49$ & 1.18 & $<1.07$ & $<0.91$ & $<0.59$ & - & - \\
\hline IRAS $18588+3517$ & 0.57 & 0.37 & 0.24 & 5.12 & $<0.40$ & $<0.43$ & 1.87 & $<3.75$ & 7.96 & $<4.91$ & $<4.13$ & $<4.05$ & - & - \\
\hline IRAS 20100-4156 & 0.33 & 0.23 & $<0.31$ & 6.73 & $<0.65$ & $<0.49$ & 1.71 & $<0.69$ & 2.86 & $<3.58$ & 1.20 & 1.34 & - & - \\
\hline IRAS 20286+1846 & $<0.56$ & $<0.50$ & $<0.27$ & 1.67 & $<0.72$ & $<0.65$ & 0.37 & $<0.30$ & 0.44 & $<0.58$ & $<0.73$ & $<0.70$ & - & - \\
\hline IRAS 21077+3358 & $<0.67$ & $<0.63$ & $<0.39$ & 3.06 & $<0.45$ & $<0.36$ & 1.09 & $<0.50$ & 0.77 & $<0.80$ & $<0.60$ & $<0.47$ & - & - \\
\hline IRAS $21272+2514$ & $<0.18$ & $<0.16$ & 0.16 & 2.29 & $<0.31$ & $<0.22$ & 0.35 & $<0.21$ & 0.45 & $<0.50$ & $<0.68$ & $<0.46$ & - & - \\
\hline IRAS 22055+3024 & 0.12 & $<0.67$ & $<0.71$ & 4.55 & $<0.76$ & $<0.74$ & 1.05 & $<0.51$ & 1.04 & $<2.12$ & $<2.30$ & $<1.34$ & - & - \\
\hline IRAS 22116+0437 & $<0.47$ & $<0.73$ & $<0.37$ & 2.25 & $<0.82$ & $<0.68$ & 1.33 & $<1.07$ & 1.04 & $<2.16$ & $<2.54$ & $<2.35$ & - & - \\
\hline IRAS $22491-1808$ & - & 0.41 & $<0.54$ & 4.88 & $<0.55$ & $<0.43$ & 1.70 & $<0.72$ & 1.86 & $<3.40$ & $<7.11$ & $<3.35$ & 13.72 & - \\
\hline IRAS $23028+0725$ & $<0.55$ & $<0.61$ & $<0.39$ & 1.81 & $<0.90$ & $<0.72$ & 1.04 & $<1.05$ & $<1.15$ & $<1.72$ & $<2.70$ & $<1.72$ & - & - \\
\hline IRAS 23233+0946 & 0.37 & 0.33 & $<0.43$ & 4.93 & $<0.43$ & $<0.38$ & 1.06 & $<0.89$ & 3.13 & $<1.06$ & 1.20 & 0.66 & - & - \\
\hline IRAS $23365+3604$ & - & $<0.36$ & $<0.51$ & 8.51 & $<0.63$ & $<0.52$ & 1.12 & $<0.44$ & 4.29 & $<4.74$ & $<11.96$ & $<7.97$ & 6.81 & - \\
\hline IRAS 00163-1039 & - & 3.40 & 0.43 & 80.95 & $<1.96$ & $<0.92$ & 14.53 & $<1.70$ & 30.85 & $<7.59$ & 1.42 & 3.35 & 34.70 & 73.84 \\
\hline IRAS 01572+0009 & 0.73 & 3.06 & $<0.72$ & 6.15 & 5.51 & $<3.23$ & 10.34 & $<0.89$ & 1.66 & 4.76 & 9.82 & $<6.12$ & - & - \\
\hline IRAS 05083+7936 & - & $<1.19$ & $<1.57$ & 49.40 & 0.60 & 0.48 & 7.63 & $<1.19$ & 18.67 & $<1.89$ & 1.28 & 1.33 & 28.00 & 71.27 \\
\hline IRAS 06538+4628 & - & 0.80 & 1.09 & 47.39 & 0.69 & $<1.16$ & 6.07 & 1.17 & 19.11 & $<2.33$ & $<6.97$ & 2.70 & 37.60 & 57.37 \\
\hline IRAS 08559+1053 & $<0.37$ & 0.56 & $<0.43$ & 8.38 & 0.51 & 0.35 & 1.87 & $<0.93$ & 1.35 & $<1.34$ & 2.54 & 0.45 & - & - \\
\hline IRAS 09437+0317 & - & $<1.04$ & $<0.61$ & 8.74 & $<0.64$ & $<0.51$ & 1.22 & $<0.70$ & 3.33 & $<0.99$ & 0.47 & 0.68 & 9.45 & 23.27 \\
\hline IRAS $10565+2448$ & - & $<0.76$ & $<1.34$ & 57.60 & $<1.29$ & 0.67 & 7.65 & $<1.02$ & 12.42 & $<3.07$ & $<5.13$ & $<2.35$ & 20.89 & 51.37 \\
\hline IRAS $11119+3257$ & $<0.83$ & 0.31 & $<0.78$ & 2.19 & $<0.71$ & $<0.58$ & 1.89 & $<2.30$ & $<1.75$ & $<2.40$ & $<3.08$ & $<1.95$ & - & - \\
\hline IRAS $13349+2438$ & 2.76 & 1.66 & $<0.45$ & 1.43 & 0.81 & $<0.99$ & 3.50 & $<4.54$ & 1.68 & 3.46 & 7.28 & $<5.11$ & - & - \\
\hline IRAS $15001+1433$ & 0.39 & 0.28 & $<0.30$ & 6.61 & 1.08 & $<0.98$ & 2.62 & $<0.78$ & 2.36 & 0.67 & 1.21 & 0.56 & - & - \\
\hline IRAS $15206+3342$ & 2.01 & 3.82 & 0.23 & 10.96 & $<0.34$ & $<0.41$ & 19.87 & $<1.03$ & 8.58 & 1.36 & 0.74 & 1.21 & - & - \\
\hline IRAS 20460+1925 & $<0.39$ & $<0.66$ & $<0.42$ & $<0.44$ & $<0.60$ & $<0.51$ & $<0.69$ & $<0.72$ & $<0.79$ & $<3.68$ & 1.95 & $<1.01$ & - & - \\
\hline IRAS 23007+0836 & - & 8.67 & $<1.84$ & 179.04 & 8.36 & $<4.23$ & 33.69 & $<3.28$ & 70.16 & 15.51 & 30.98 & 9.47 & 97.56 & 188.36 \\
\hline IRAS 23394-0353 & - & 0.72 & 0.57 & 46.75 & $<1.45$ & $<1.08$ & 7.71 & $<1.10$ & 17.16 & $<1.92$ & 1.27 & 2.43 & 44.29 & 53.48 \\
\hline IRAS $23498+2423$ & 0.23 & 1.27 & $<0.23$ & 3.10 & 0.92 & $<0.91$ & 7.79 & $<0.55$ & 1.13 & 1.26 & 4.61 & $<3.77$ & - & - \\
\hline
\end{tabular}

Note. Line fluxes are given in $10^{-21} \mathrm{~W} \mathrm{~cm}^{-2}$. The symbol “_” indicates that the redshifted line wavelength lay outside the range of the IRS. 
Table 5

Molecular $\mathrm{H}_{2}$ Gas Properties

\begin{tabular}{|c|c|c|c|c|c|c|c|c|c|c|c|}
\hline $\begin{array}{c}\text { Object } \\
\lambda_{\text {rest }}(\mu \mathrm{m})\end{array}$ & $\begin{array}{l}\mathrm{H}_{2} \mathrm{~S}(7) \\
5.51 \mu \mathrm{m}\end{array}$ & $\begin{array}{l}\mathrm{H}_{2} \mathrm{~S}(5) \\
6.91 \mu \mathrm{m} \\
\end{array}$ & $\begin{array}{l}\mathrm{H}_{2} \mathrm{~S}(4) \\
8.03 \mu \mathrm{m}\end{array}$ & $\begin{array}{l}\mathrm{H}_{2} \mathrm{~S}(3) \\
9.67 \mu \mathrm{m}\end{array}$ & $\begin{array}{c}\mathrm{H}_{2} \mathrm{~S}(2) \\
12.28 \mu \mathrm{m}\end{array}$ & $\begin{array}{c}\mathrm{H}_{2} S(1) \\
17.04 \mu \mathrm{m}\end{array}$ & $\begin{array}{c}\mathrm{H}_{2} \mathrm{~S}(0) \\
28.22 \mu \mathrm{m}\end{array}$ & $\begin{array}{c}T_{\text {warm }} \\
(\mathrm{K})\end{array}$ & $\begin{array}{l}T_{\text {hot }} \\
(\mathrm{K})\end{array}$ & $\begin{array}{c}M_{\text {warm }} \\
\left(10^{7} M_{\odot}\right)\end{array}$ & $\begin{array}{c}M_{\mathrm{hot}} \\
\left(10^{7} M_{\odot}\right) \\
\end{array}$ \\
\hline IRAS 01355-1814 & $<1.52$ & $<3.25$ & - & 0.22 & 0.63 & 1.13 & $<1.63$ & 262 & & 4.74 & \\
\hline IRAS $01418+1651$ & $<5.29$ & $6.96:$ & - & 1.16 & 0.94 & 2.15 & $<5.40$ & 320 & & 0.14 & \\
\hline IRAS $01562+2528$ & $<3.25$ & $<7.06$ & - & $<0.52$ & $<0.39$ & 0.94 & $<0.92$ & & & & \\
\hline IRAS $02524+2046$ & $<1.52$ & 0.88 : & - & $<2.51$ & $<0.46$ & 0.88 & $<1.03$ & & & & \\
\hline IRAS $03521+0028$ & $<2.89$ & $0.58:$ & - & 0.63 & 0.47 & 1.69 & $<0.55$ & 292 & & 4.24 & \\
\hline IRAS $04121+0223$ & $<3.54$ & $1.03:$ & - & $<1.62$ & $<0.43$ & $<0.73$ & $<1.06$ & & & & \\
\hline IRAS $04454-4838$ & $<3.31$ & $<13.24$ & - & 1.04 & 1.27 & 3.05 & 0.76 & 222 & & 0.82 & \\
\hline IRAS 06487+2208 & $<5.99$ & $0.97:$ & - & 2.10 & 1.02 & 1.80 & $<1.52$ & 381 & & 4.13 & \\
\hline IRAS $07163+0817$ & $<1.84$ & $<4.58$ & - & $<0.77$ & 0.04 & 0.47 & 1.68 & 110 & & 0.60 & \\
\hline IRAS $07572+0533$ & $<1.03$ & $<2.62$ & - & $<0.90$ & $<0.50$ & $<0.99$ & $<3.41$ & & & & \\
\hline IRAS 08201+2801 & $<3.41$ & $<10.84$ & - & 0.28 & $<0.90$ & 0.56 & $<0.91$ & 313 & & 1.79 & \\
\hline IRAS 08449+2332 & $<3.67$ & $1.22:$ & - & 0.63 & 0.33 & 1.36 & $<0.70$ & 301 & & 3.48 & \\
\hline IRAS 08474+1813 & $<1.07$ & $0.61:$ & - & 0.75 & $<0.46$ & 0.27 & $<1.33$ & 493 & & 0.68 & \\
\hline IRAS 09039+0503 & 1.13 & $2.69:$ & - & 2.21 & 1.46 & 2.91 & 1.27 & 198 & 978 & 4.88 & 0.42 \\
\hline IRAS 09539+0857 & $<2.40$ & $<6.29$ & - & 0.53 & 0.26 & 1.07 & $<0.95$ & 308 & & 1.93 & \\
\hline IRAS $10035+2740$ & $<0.98$ & $0.81:$ & - & 0.70 & $<0.63$ & 1.06 & $<2.31$ & 334 & & 3.29 & \\
\hline IRAS $10039-3338$ & $<28.84$ & $<46.95$ & - & 3.27 & 1.71 & 3.95 & $<7.46$ & 348 & & 0.46 & \\
\hline IRAS $10173+0828$ & $<5.73$ & $3.00:$ & - & 0.58 & 0.49 & 1.38 & $<1.67$ & 302 & & 0.33 & \\
\hline IRAS $10339+1548$ & $<1.09$ & $<2.88$ & - & $<0.58$ & $<0.42$ & 0.42 & $<1.94$ & & & & \\
\hline IRAS $10378+1109$ & 0.53 & $1.42:$ & - & 1.82 & 0.55 & 2.25 & $<0.61$ & 315 & 876 & 4.59 & 0.46 \\
\hline IRAS $10485-1447$ & 0.52 & $<5.57$ & - & 0.18 & 0.14 & 0.28 & $<0.76$ & 307 & 1540 & 0.55 & 0.03 \\
\hline IRAS $11028+3130$ & $<0.44$ & $<1.83$ & - & $<0.61$ & $<0.36$ & 0.39 & $<1.75$ & & & & \\
\hline IRAS $11180+1623$ & $<2.25$ & $0.51:$ & - & 0.39 & 0.61 & 1.06 & $<1.88$ & 302 & & 3.32 & \\
\hline IRAS $11524+1058$ & $<1.99$ & $<4.35$ & - & $<0.43$ & $<0.42$ & 0.66 & $<2.14$ & & & & \\
\hline IRAS 12018+1941 & $<3.27$ & $<8.84$ & - & 0.47 & 0.36 & 1.28 & 2.21 & 165 & & 4.10 & \\
\hline IRAS $12032+1707$ & $<2.78$ & $4.93:$ & $<0.27$ & 0.81 & 0.58 & 1.61 & $<3.34$ & 312 & & 9.24 & \\
\hline IRAS $12112+0305$ & 7.03 & $5.89:$ & - & 1.87 & 1.66 & 3.71 & 1.30 & 195 & 1680 & 2.01 & 0.08 \\
\hline IRAS $12540+5708$ & $<32.27$ & $<49.24$ & - & 2.42 & 3.21 & 6.23 & $<17.62$ & 301 & & 1.07 & \\
\hline IRAS $13218+0552$ & $<4.32$ & $<7.55$ & - & 0.32 & 0.42 & 0.97 & $<2.91$ & 292 & & 4.83 & \\
\hline IRAS $13428+5608$ & $<13.14$ & 8.96 : & - & 7.77 & 4.83 & 8.63 & $<9.82$ & 360 & & 1.19 & \\
\hline IRAS $13451+1232$ & $<8.46$ & $3.26:$ & - & 1.65 & 1.10 & 2.61 & $<0.79$ & 329 & & 4.18 & \\
\hline IRAS $14059+2000$ & 0.91 & $1.55:$ & - & 2.12 & 0.73 & 2.36 & $<0.88$ & 319 & 944 & 3.90 & 0.40 \\
\hline IRAS $14070+0525$ & $<2.54$ & $<5.85$ & $<0.41$ & 0.28 & 0.22 & 1.30 & $<0.85$ & 260 & & 11.42 & \\
\hline IRAS $14553+1245$ & 0.32 & $<9.92$ & - & 0.92 & 0.19 & 1.05 & $<0.82$ & 317 & 906 & 1.76 & 0.19 \\
\hline IRAS $15327+2340$ & 22.70 & $41.6:$ & - & $<0.56$ & 7.66 & 13.68 & 14.53 & 159 & & 0.42 & \\
\hline IRAS $16090-0139$ & 1.20 & $<15.12$ & - & 1.10 & $<0.28$ & 2.12 & $<2.93$ & 299 & 1160 & 4.09 & 0.20 \\
\hline IRAS $16255+2801$ & $<1.82$ & 0.21 : & - & $<0.59$ & 0.28 & 0.55 & $<4.18$ & 348 & & 1.07 & \\
\hline IRAS $16300+1558$ & $<1.77$ & $<5.63$ & 0.31 & 0.56 & 0.42 & 1.58 & $<1.31$ & 287 & & 11.33 & \\
\hline IRAS $17207-0014$ & 6.12 & $10.2:$ & - & 4.56 & 4.44 & 7.51 & $<3.60$ & 311 & 1230 & 1.30 & 0.07 \\
\hline IRAS $18368+3549$ & $<6.98$ & $4.22:$ & - & 1.11 & 0.70 & 1.38 & $<0.81$ & 349 & & 1.95 & \\
\hline IRAS $18588+3517$ & $<7.44$ & $<15.54$ & - & 0.92 & 0.76 & 1.61 & $<5.12$ & 324 & & 1.89 & \\
\hline IRAS $20100-4156$ & $<9.04$ & $<15.77$ & - & 0.78 & 0.35 & 0.92 & 0.94 & 195 & & 1.61 & \\
\hline IRAS $20286+1846$ & $<1.74$ & 0.35 : & - & $<0.78$ & 0.30 & 0.92 & $<0.51$ & 282 & & 1.80 & \\
\hline IRAS $21077+3358$ & $<2.13$ & $1.59:$ & - & 0.67 & 0.34 & 1.16 & $<1.29$ & 319 & & 4.05 & \\
\hline IRAS $21272+2514$ & 0.39 & $0.51:$ & - & 0.36 & 0.28 & 0.91 & $<0.46$ & 287 & 1160 & 2.22 & 0.09 \\
\hline IRAS $22055+3024$ & 0.65 & $2.52:$ & - & 1.30 & 1.09 & 1.61 & $<1.39$ & 320 & 976 & 2.72 & 0.24 \\
\hline IRAS $22116+0437$ & $<2.45$ & $<6.21$ & - & 0.74 & 0.64 & 1.09 & $<1.82$ & 340 & & 4.70 & \\
\hline IRAS 22491-1808 & $<7.01$ & $<14.81$ & - & 0.76 & 0.92 & 2.10 & $<8.22$ & 298 & & 1.23 & \\
\hline IRAS $23028+0725$ & - & - & - & 0.48 & 0.45 & 0.77 & $<1.67$ & 333 & & 1.86 & \\
\hline IRAS $23233+0946$ & $<3.20$ & $1.60:$ & - & 1.03 & 0.80 & 1.47 & $<0.73$ & 340 & & 2.53 & \\
\hline IRAS $23365+3604$ & $<7.02$ & $6.98:$ & - & 1.26 & 0.73 & 2.14 & $<5.75$ & 321 & & 0.84 & \\
\hline IRAS 00163-1039 & $<9.00$ & $24.46:$ & - & 2.79 & 2.87 & 5.13 & $<2.63$ & 326 & & 0.32 & \\
\hline IRAS 01572+0009 & 2.15 & $1.21:$ & - & 0.60 & $<0.71$ & 2.15 & $<1.36$ & 268 & 1650 & 6.26 & 0.15 \\
\hline IRAS 05083+7936 & $<8.11$ & 27.31: & - & 2.34 & 2.73 & 3.59 & $<2.75$ & 341 & & 0.99 & \\
\hline IRAS $06538+4628$ & $<3.69$ & 14.56: & - & $<0.63$ & 3.28 & 8.86 & 2.63 & 189 & & 0.38 & \\
\hline IRAS 08559+1053 & $<5.91$ & $1.76:$ & - & 0.72 & 0.64 & 1.83 & $<0.74$ & 298 & & 4.44 & \\
\hline IRAS $09437+0317$ & $<9.39$ & $22.60:$ & - & $<0.36$ & 0.68 & 2.68 & 2.20 & 153 & & 0.11 & \\
\hline IRAS $10565+2448$ & $<12.33$ & $32.87:$ & - & 3.34 & 1.95 & 5.73 & $<3.77$ & 320 & & 1.06 & \\
\hline IRAS $11119+3257$ & $<6.58$ & $<7.87$ & - & 0.42 & $<1.02$ & 2.47 & $<2.23$ & 256 & & 10.21 & \\
\hline IRAS $13349+2438$ & $<23.79$ & $<20.52$ & - & $<1.67$ & $<0.83$ & $<1.05$ & $<1.62$ & & & & \\
\hline IRAS $15001+1433$ & $<4.21$ & 1.72 : & - & 0.44 & 0.24 & 1.25 & $<0.84$ & 283 & & 3.72 & \\
\hline IRAS $15206+3342$ & $<8.31$ & $1.61:$ & - & 0.65 & 0.46 & 0.94 & 1.02 & 196 & & 1.54 & \\
\hline IRAS $20460+1925$ & - & - & - & $<0.48$ & $<0.43$ & $<0.88$ & $<4.24$ & & & & \\
\hline
\end{tabular}


Table 5

(Continued)

\begin{tabular}{|c|c|c|c|c|c|c|c|c|c|c|c|}
\hline $\begin{array}{c}\text { Object } \\
\lambda_{\text {rest }}(\mu \mathrm{m})\end{array}$ & $\begin{array}{l}\mathrm{H}_{2} S(7) \\
5.51 \mu \mathrm{m}\end{array}$ & $\begin{array}{l}\mathrm{H}_{2} S(5) \\
6.91 \mu \mathrm{m} \\
\end{array}$ & $\begin{array}{l}\mathrm{H}_{2} S(4) \\
8.03 \mu \mathrm{m} \\
\end{array}$ & $\begin{array}{l}\mathrm{H}_{2} S(3) \\
9.67 \mu \mathrm{m} \\
\end{array}$ & $\begin{array}{c}\mathrm{H}_{2} S(2) \\
12.28 \mu \mathrm{m} \\
\end{array}$ & $\begin{array}{c}\mathrm{H}_{2} S(1) \\
17.04 \mu \mathrm{m}\end{array}$ & $\begin{array}{c}\mathrm{H}_{2} S(0) \\
28.22 \mu \mathrm{m}\end{array}$ & $\begin{array}{c}T_{\text {warm }} \\
(\mathrm{K})\end{array}$ & $\begin{array}{l}T_{\text {hot }} \\
(\mathrm{K})\end{array}$ & $\begin{array}{c}M_{\text {warm }} \\
\left(10^{7} M_{\odot}\right) \\
\end{array}$ & $\begin{array}{c}M_{\mathrm{hot}} \\
\left(10^{7} M_{\odot}\right)\end{array}$ \\
\hline IRAS 23007+0836 & $<23.20$ & $77.35:$ & - & $<3.53$ & 6.30 & 12.90 & $<6.28$ & 342 & & 0.27 & \\
\hline IRAS 23394-0353 & $<14.60$ & $32.52:$ & - & 3.15 & 2.44 & 5.24 & 1.79 & 226 & & 0.23 & \\
\hline IRAS $23498+2423$ & $<1.25$ & $0.49:$ & - & 0.34 & $<0.29$ & 0.89 & $<1.50$ & 298 & & 4.66 & \\
\hline
\end{tabular}

Notes. Line fluxes are given in $10^{-21} \mathrm{~W} \mathrm{~cm}^{-2}$. The symbol “-” indicates that the redshifted line lay outside of the IRS spectral range. Fluxes for $\mathrm{H}_{2} S(7)$ and $S(5)$ are measured in the SL module; all other lines are measured in the SH and LH modules. $S(5)$ lines are tentative upper limits (indicated by a colon (:)) due to possible blending with [Ar II] at $6.99 \mu \mathrm{m}$; see Section 5.3.

[Ar II] and the $S(5)$ emission (see Section 5.5.2). Emission in the [Ar II] $/ \mathrm{H}_{2} \mathrm{~S}(5)$ complex is seen in more than half of our sample, however, and so we present measurements for the entire feature, including blended emission from both lines. We caution that these fluxes should be viewed as upper limits for either [Ar II] or $\mathrm{H}_{2} \mathrm{~S}(5)$ emission, since the SL module does not have sufficient resolution to separate the two features.

For galaxies in which multiple $\mathrm{H}_{2}$ lines are observed, we fit excitation temperatures $\left(T_{\mathrm{ex}}\right)$ to the molecular gas following the methods of Rigopoulou et al. (2002) and Higdon et al. (2006). We assume that the emission is optically thin (so that the lines are unsaturated), populations are in local thermodynamic equilibrium (LTE), and that the sources are unresolved in the Spitzer beam. The luminosity of a molecular emission line for the transition from $(J+2) \rightarrow J$ is then $L_{J}=A_{J} \times \Delta E_{J} \times N_{J+2}$, where $A_{J}$ is the Einstein-A coefficient, $\Delta E_{J}$ is the energy of the transition, and $N_{J+2}$ is the number of molecules in the $J+2$ state. The partition function for a given symmetry branch is

$$
Z_{J_{o / p}}=\sum_{J_{o / p}} g_{J} \exp \left[-\mathrm{E}_{\mathrm{J}} / \mathrm{kT}_{\mathrm{ex}}\right]
$$

where $T_{\mathrm{ex}}$ is the excitation temperature and we sum only over a single symmetry branch (ortho or para). The statistical weights are $g_{J}=(2 J+1) \times J_{s}$, where $J_{s}=1$ for the para branch (even $J)$ and $J_{s}=3$ for ortho (odd $J$ ).

Assuming that the lines are in LTE, the ratio of level populations follows a Boltzmann distribution such that $N_{J} \propto$ $g_{J} \exp \left[-E_{J} / k T_{\mathrm{ex}}\right]$. The inverse slope of the best-fit line of an excitation diagram yields $T_{\mathrm{ex}}$-Figure 6 shows examples of temperature fits for our data. The total warm $\mathrm{H}_{2}$ mass can be then calculated from $T_{\mathrm{ex}}$ and the flux $\left(F_{J}\right)$ from any transition as

$$
M_{\mathrm{tot}}=m_{\mathrm{H}_{2}} \times \phi_{o / p} \times \frac{\left(4 \pi D_{L}^{2}\right) F_{J} Z_{J_{o / p}}}{A_{J} \Delta E_{J} g_{J} \exp \left[-E_{J} / k T_{\mathrm{ex}}\right]},
$$

where $\phi_{o / p}$ is a numerical factor accounting for the ortho-topara ratio (assumed to be 3:1), $m_{\mathrm{H}_{2}}$ is the mass of the hydrogen molecule, and $D_{L}$ is the luminosity distance.

For cases where the $S(7)$ line was detected, a single excitation temperature gives a poor fit to the full set of transitions. In these cases, we first fit $T_{\mathrm{ex}}$ between $S(3)$ and $S(7)$, measuring hotter gas. We then subtracted this component from the $S(0)$ to $S(3)$ fluxes, and fit a second $T_{\mathrm{ex}}$ to the warm gas component. This decreased the mean warm $T_{\text {ex }}$ by $\sim 20 \mathrm{~K}$, with a negligible effect on the gas mass. We calculated the warm $\mathrm{H}_{2}$ mass using the flux in the $S(1)$ transition and the hot gas mass using the $S(3)$ flux (Table 5).

Both Higdon et al. (2006) and Roussel et al. (2007) suggest that the $\mathrm{H}_{2}$ emission arises from far-ultraviolet photons from massive stars powering photodissociation regions (PDRs). Detections of the $\mathrm{H}_{2} \mathrm{~S}(3)$ transition in nearly all objects implies that the silicate absorption at $9.7 \mu \mathrm{m}$ must be partially background to the warm molecular gas seen in emission. Since the dust is very optically thick in almost all ULIRGs, this means that at least some molecular gas (and possibly other atomic transitions) actually come from superficial layers at the edge of the merging system. Given that the OHM is typically formed within the central kiloparsec of the host galaxy, a link between the observed warm $\mathrm{H}_{2}$ gas and the OHM is uncertain.

Eleven objects in the OHM sample and two non-masing galaxies have $\mathrm{CO}$ detections published in the literature (Solomon et al. 1997; Gao \& Solomon 2004a). The beam width used for CO observations is several times that of the HR slits; since most ULIRGs are unresolved in the Spitzer beam, we consider the gas mass estimates to be comparable. The cold gas masses derived using a ULIRG-calibrated $M_{\mathrm{H}_{2}} / L_{\mathrm{CO}}$ ratio of $\sim 1.4 M_{\odot} /\left(\mathrm{K} \mathrm{km} \mathrm{s}^{-1} \mathrm{pc}^{2}\right)$ give a warm gas mass fraction for the OHMs ranging from $0.04 \%$ to $0.8 \%$, with the gas fraction of the non-masing galaxies lying in a similar range $(0.06 \%-0.1 \%)$. This is comparable to warm gas fractions in ULIRGs from Higdon et al. (2006), implying that the mid-IR $\mathrm{H}_{2}$ lines probe only a small amount of the total gas mass in these galaxies. The bulk of the remaining portion is likely cold gas without sufficient energy to excite rotational transitions in the mid-IR.

\subsection{PAH Emission}

In addition to the atomic and simple molecular emission lines, we also observed multiple features attributed to PAHs; the broad-line emission comes from vibrational modes of $\mathrm{C}-\mathrm{C}$ and $\mathrm{C}-\mathrm{H}$ bonds (Draine 2003). PAH features are ubiquitous in the mid-IR emission of starburst galaxies and ULIRGs (Lutz et al. 1998; Genzel et al. 1998; Sturm et al. 2000; Peeters et al. 2004; Desai et al. 2007; Imanishi et al. 2007), and dominate the LR spectra of most galaxies in our sample. Multiple PAH features are seen for all OHMs, encompassing galaxies with very wide ranges in continuum shape and line emission. We detect strong PAH transitions centered at 6.2, 7.7, 8.6, 11.3, and $12.7 \mu \mathrm{m}$, several of which are also visible in the HR spectra. Weaker emission features at 13.5, 14.2, 16.4, 17.1, and $17.4 \mu \mathrm{m}$ are also visible in many galaxies.

We measured the PAH emission via two methods: the first defines a local continuum around the PAH feature using a spline fit and then integrates the total flux after baseline subtraction. The default continuum pivots are located at 5.15, 5.55, 5.95, 6.55 , and $7.10 \mu \mathrm{m}$ for the $6.2 \mu \mathrm{m}$ feature and at $10.1,10.9$, 11.8 , and $12.4 \mu \mathrm{m}$ for the $11.3 \mu \mathrm{m}$ feature. These are shifted slightly for each object to avoid both broad absorption features (including water ice and hydrocarbons) and narrow atomic emission lines. We quantify the emission from the two cleanest 

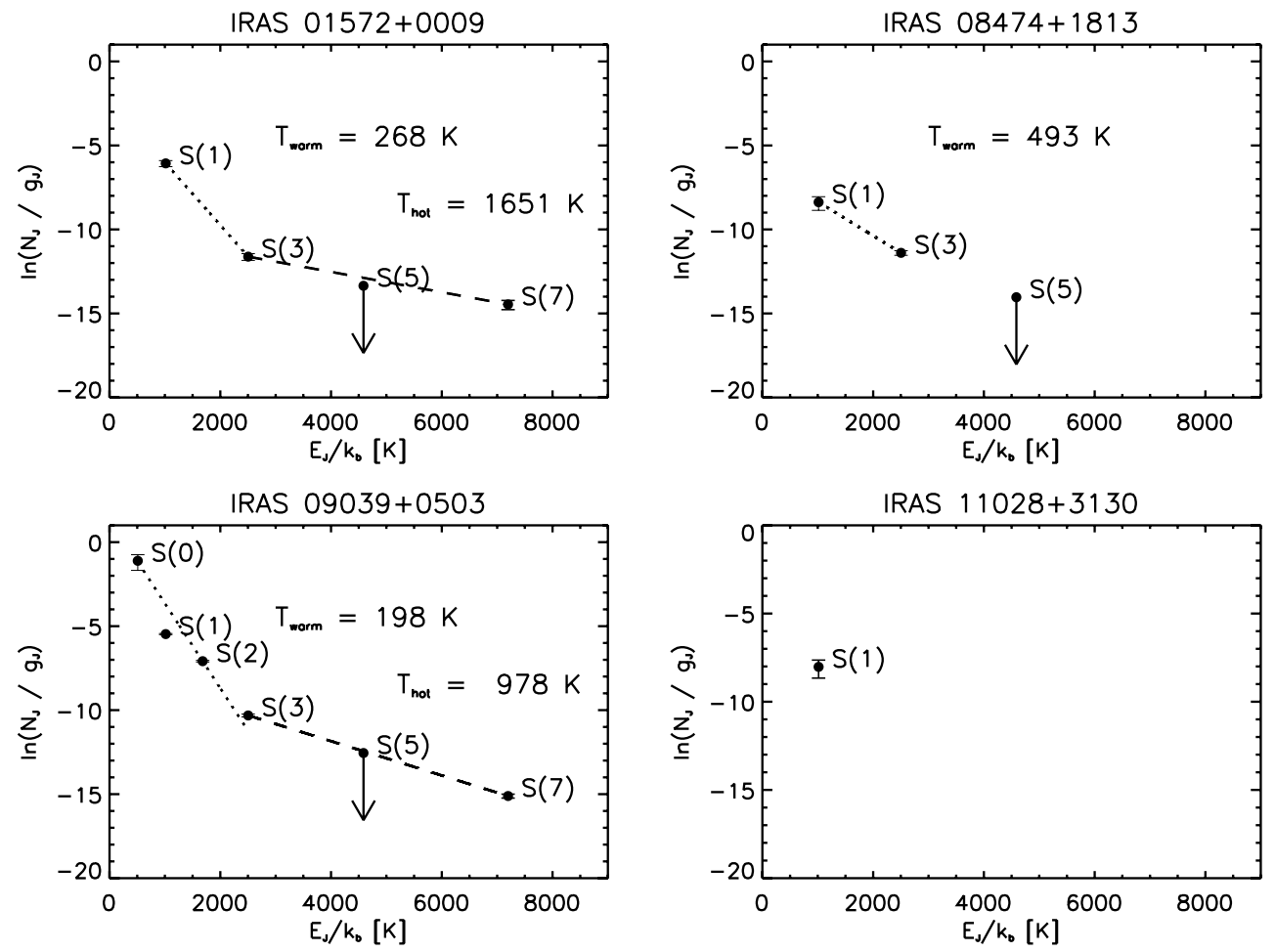

Figure 6. Example: $\mathrm{H}_{2}$ excitation diagrams for both non-masing (IRAS 01572+0009) and OHM galaxies (all others). Two galaxies (left) are fit with both warm and hot excitation temperatures; IRAS $08474+1813$ fits only a warm component since the higher $J$ lines are not detected. IRAS $11028+3130$ shows an example of a galaxy with only a single $\mathrm{H}_{2}$ detection (for which no $T_{\mathrm{ex}}$ can be determined). Dotted lines are fit to the warm gas for all detections from $S(0)$ to $S(3)$; dashed lines are fit to the hotter gas using detections of $S(3), S(4)$, and $S(7)$. The $S(5)$ line is always an upper limit due to possible blending from [Ar II] and is not used in the temperature fits.

PAH features appearing in our spectra: the 6.2 and $11.3 \mu \mathrm{m}$ complexes (Table 6).

A second method uses PAHFIT (Smith et al. 2007), a public IDL package, to fit global mid-IR spectral templates and simultaneously measure the relative effects of overlapping features. The routine decomposes the LR IRS spectra into emission from stellar continuum, dust features (including PAHs), atomic and molecular lines, and blackbodies from thermally heated dust at a variety of temperatures; this is simultaneously fit with an extinction curve including silicate features at 9.7 and $18 \mu \mathrm{m}$. Due to the large number of parameters being fit, however, even strong features may overlap sufficiently to affect the accuracy of the fit (Spoon et al. 2002); in addition, PAHFIT does not fit for several features commonly found in ULIRGs, such as absorption features from ice, hydrocarbons, and gas-phase molecules.

PAHFIT fits the dust emission features (including PAHs) with a Drude profile, which has the form

$$
I_{\lambda}[\lambda]=\frac{b \gamma^{2}}{\left(\lambda / \lambda_{c}-\lambda_{c} / \lambda\right)^{2}+\gamma^{2}},
$$

where $\lambda_{c}$ is the central wavelength, $\gamma$ is the fractional FWHM, and $b$ is the (peak) central intensity. The Drude profile is typically broader than a Gaussian, with significant amounts of power in the extended wings. Several dust emission features (e.g., the 7.7 and $12.7 \mu \mathrm{m}$ PAHs) require more than one component for a reasonable fit. PAHFIT returned positive detections for both the 6.2 and $11.3 \mu \mathrm{m}$ PAH features for nearly all galaxies; the fit for OHM IRAS $07572+0533$ showed no emission at $6.2 \mu \mathrm{m}$, while the fits to OHM IRAS $13218+0552$ and the non-masing galaxy IRAS $13349+2438$ show no emission in either dust feature.

We compared the flux measured in the 6.2 and $11.3 \mu \mathrm{m}$ $\mathrm{PAH}$ features from our baseline-subtracted spline fits to the
PAHFIT values; results from PAHFIT are consistently higher than those from the spline fit, indicating significant mixing between the PAH emission and what was previously designated as "continuum" (Figure 7). Fluxes of the $6.2 \mu \mathrm{m}$ complex measured with PAHFIT are a factor of $\sim 3-4$ greater than the spline-fit fluxes, while the $11.3 \mu \mathrm{m}$ feature is an average of $\sim 2-3$ times larger. The relative strengths of the two features are consistent using both methods; the mean value of the $(6.2 \mu \mathrm{m}$ $\mathrm{PAH} / 11.3 \mu \mathrm{m} \mathrm{PAH}$ ) ratio is identical to within $15 \%$ for OHMs.

Galliano et al. (2008) also use both spline and multicomponent profile fitting approaches to measure the PAH variations within galaxies; they show that both methods yield the same overall trends, although each have their own underlying biases depending on the property being measured. Given the large contributions of overlapping dust emission features to the 5-10 $\mu \mathrm{m}$ spectrum, which are not possible to separate from the underlying blackbody emission using spline fits (Marshall et al. 2007), we consider the PAHFIT results to be the more robust method. Measurements of PAH features in the literature, however, typically use a spline-fit method (e.g., Brandl et al. 2006; Desai et al. 2007; Spoon et al. 2007; Zakamska et al. 2008). In particular, comparisons of PAH data from the OHM galaxies to other samples (e.g., the "fork diagram" from Spoon et al. 2007) must use the same method to return physically meaningful results. While PAHFIT fluxes may thus better represent the absolute PAH luminosity, the spline-fit data are used when comparing the OHMs to objects from the literature (Table 6).

Water ice absorption at $6 \mu \mathrm{m}$ can have significant effects on the measurement of the $6.2 \mu \mathrm{m}$ PAH EW; following the method of Spoon et al. (2007), we correct for this by substituting the continuum inferred while measuring the $9.7 \mu \mathrm{m}$ silicate strength for the measured $6.2 \mu \mathrm{m}$ continuum (see Section 5.5). In total, $24 \mathrm{OHMs}$ and three non-masing galaxies with SL data 
Table 6

PAH Emission Features in Low-resolution Spectra

\begin{tabular}{|c|c|c|c|c|c|c|c|c|c|c|}
\hline \multirow[t]{2}{*}{ Object } & \multicolumn{2}{|c|}{ PAHFIT Luminosity } & \multicolumn{3}{|c|}{ PAHFIT EW } & \multicolumn{2}{|c|}{ Spline-fit Luminosity } & \multicolumn{3}{|c|}{ Spline-fit EW } \\
\hline & $\begin{array}{c}6.2 \\
\left(\log L / L_{\odot}\right)\end{array}$ & $\begin{array}{c}11.3 \\
\left(\log L / L_{\odot}\right)\end{array}$ & $\begin{array}{c}6.2 \\
(\mu \mathrm{m})\end{array}$ & $\begin{array}{c}6.2 \text { ice } \\
(\mu \mathrm{m})\end{array}$ & $\begin{array}{l}11.3 \\
(\mu \mathrm{m})\end{array}$ & $\begin{array}{c}6.2 \\
\left(\log L / L_{\odot}\right)\end{array}$ & $\begin{array}{c}11.3 \\
\left(\log L / L_{\odot}\right)\end{array}$ & $\begin{array}{c}6.2 \\
(\mu \mathrm{m})\end{array}$ & $\begin{array}{c}6.2 \text { ice } \\
(\mu \mathrm{m})\end{array}$ & $\begin{array}{l}11.3 \\
(\mu \mathrm{m})\end{array}$ \\
\hline IRAS $01355-1814$ & 9.81 & 9.42 & 2.25 & & 0.49 & 9.07 & 8.97 & 0.14 & & 0.26 \\
\hline IRAS $01418+1651$ & 8.98 & 9.07 & 1.81 & & 1.86 & 8.60 & 8.48 & 0.44 & & 0.61 \\
\hline IRAS $01562+2528$ & 9.59 & 9.71 & 2.07 & & 2.22 & 9.23 & 9.43 & 0.37 & & 0.86 \\
\hline IRAS $02524+2046$ & 9.37 & 9.62 & 0.54 & & 1.85 & 9.19 & 9.13 & 0.49 & & 0.53 \\
\hline IRAS $03521+0028$ & 9.78 & 9.60 & 1.67 & 0.36 & 0.81 & 9.41 & 9.29 & 0.43 & 0.36 & 0.60 \\
\hline IRAS $04121+0223$ & 9.48 & 9.42 & 1.82 & 0.89 & 2.01 & 9.11 & 9.02 & 0.44 & 0.38 & 0.78 \\
\hline IRAS $04454-4838$ & 10.07 & 9.90 & 23.53 & 0.05 & 1.31 & 8.26 & 8.42 & 0.07 & 0.05 & 1.07 \\
\hline IRAS 06487+2208 & 9.89 & 9.75 & 0.55 & & 0.36 & 9.60 & 9.47 & 0.26 & & 0.26 \\
\hline IRAS $07163+0817$ & 9.21 & 9.12 & 12.82 & & 1.30 & 8.93 & 8.84 & 0.58 & & 0.77 \\
\hline IRAS $07572+0533$ & - & 8.95 & - & & 0.04 & $<8.95$ & 8.88 & $<0.10$ & & 0.04 \\
\hline IRAS 08201+2801 & 10.03 & 9.52 & 2.83 & 0.39 & 0.57 & 9.41 & 9.33 & 0.19 & 0.09 & 0.71 \\
\hline IRAS 08449+2332 & 9.78 & 9.63 & 2.79 & & 1.09 & 9.38 & 9.25 & 0.43 & & 0.59 \\
\hline IRAS $08474+1813$ & 9.37 & 9.20 & 2.33 & & 1.41 & 8.72 & 8.82 & 0.23 & & 1.40 \\
\hline IRAS 09039+0503 & 9.65 & 9.73 & 1.59 & 0.19 & 2.58 & 9.13 & 9.09 & 0.30 & 0.19 & 0.76 \\
\hline IRAS 09539+0857 & 10.2 & 10.06 & 6.38 & & 6.50 & 8.91 & 8.96 & 0.11 & & 1.09 \\
\hline IRAS $10035+2740$ & 9.21 & 9.33 & 2.10 & & 1.03 & 8.77 & 8.72 & 0.27 & & 0.25 \\
\hline IRAS $10039-3338$ & 10.96 & 10.32 & 2.90 & 0.01 & 8.28 & 8.72 & 8.94 & 0.01 & 0.01 & 0.72 \\
\hline IRAS $10173+0828$ & 9.37 & 9.51 & 2.50 & & 5.13 & 8.65 & 8.64 & 0.35 & & 0.95 \\
\hline IRAS $10339+1548$ & 9.30 & 9.63 & 0.71 & & 0.85 & 9.14 & 9.23 & 0.42 & & 0.44 \\
\hline IRAS $10378+1109$ & 9.30 & 9.36 & 0.45 & 0.04 & 0.64 & 8.62 & 9.02 & 0.07 & 0.04 & 0.49 \\
\hline IRAS $10485-1447$ & 9.73 & 9.19 & 1.64 & & 0.47 & 8.73 & 8.84 & 0.09 & 0.08 & 0.36 \\
\hline IRAS $11028+3130$ & 9.11 & 9.54 & 1.13 & & 2.33 & 8.41 & 8.85 & 0.14 & & 0.64 \\
\hline IRAS $11180+1623$ & 9.69 & 9.44 & 5.87 & 0.97 & 0.95 & 9.02 & 9.07 & 0.24 & 0.26 & 0.57 \\
\hline IRAS $11524+1058$ & 9.55 & 9.85 & 1.09 & & 3.07 & 8.87 & 9.17 & 0.12 & & 0.69 \\
\hline IRAS 12018+1941 & 9.85 & 9.44 & 0.25 & & 0.08 & 9.58 & 9.06 & 0.17 & & 0.05 \\
\hline IRAS $12032+1707$ & 10.33 & 10.20 & 3.54 & 0.06 & 1.33 & 9.40 & 9.64 & 0.07 & 0.06 & 0.61 \\
\hline IRAS $12112+0305$ & 9.62 & 9.35 & 4.39 & 0.37 & 0.76 & 9.27 & 9.02 & 0.61 & 0.37 & 0.52 \\
\hline IRAS $12540+5708$ & 9.59 & 8.66 & 0.03 & & 0.01 & 8.94 & 9.29 & 0.01 & & 0.04 \\
\hline IRAS $13218+0552$ & - & - & - & - & - & $<9.79$ & 9.50 & $<0.01$ & & $<0.03$ \\
\hline IRAS $13428+5608$ & 9.55 & 9.45 & 0.52 & 0.09 & 0.76 & 9.03 & 8.98 & 0.14 & 0.09 & 0.37 \\
\hline IRAS $13451+1232$ & 9.34 & 9.34 & 0.06 & & 0.04 & 8.23 & 8.76 & 0.01 & & 0.01 \\
\hline IRAS $14059+2000$ & 9.16 & 9.06 & 0.25 & & 0.48 & 8.93 & 8.89 & 0.23 & & 0.43 \\
\hline IRAS $14070+0525$ & 10.37 & 10.31 & 1.86 & 0.01 & 2.41 & 8.92 & 9.63 & 0.02 & 0.01 & 0.83 \\
\hline IRAS $14553+1245$ & 9.57 & 9.47 & 0.63 & & 0.65 & 9.37 & 9.22 & 0.44 & & 0.60 \\
\hline IRAS $15327+2340$ & 10.45 & 10.03 & 91.96 & 0.16 & 8.23 & 8.89 & 8.67 & 0.30 & 0.17 & 0.64 \\
\hline IRAS $16090-0139$ & 10.17 & 9.99 & 1.14 & 0.07 & 1.45 & 9.34 & 9.38 & 0.09 & 0.07 & 0.51 \\
\hline IRAS $16255+2801$ & 9.54 & 9.14 & 0.94 & 0.50 & 1.02 & 8.95 & 8.58 & 0.16 & 0.13 & 0.37 \\
\hline IRAS $16300+1558$ & 10.31 & 9.97 & 1.66 & 0.04 & 1.09 & 9.28 & 9.48 & 0.07 & 0.04 & 0.60 \\
\hline IRAS $17207-0014$ & 10.01 & 9.86 & 3.58 & 0.45 & 2.75 & 9.52 & 9.24 & 0.50 & 0.45 & 0.76 \\
\hline IRAS $18368+3549$ & 9.82 & 9.74 & 2.43 & & 2.62 & 9.50 & 9.26 & 0.61 & & 0.74 \\
\hline IRAS $18588+3517$ & 9.84 & 9.67 & 1.85 & 0.56 & 1.65 & 9.45 & 9.25 & 0.41 & 0.23 & 0.82 \\
\hline IRAS 20100-4156 & 9.96 & 9.83 & 1.52 & 0.06 & 1.23 & 9.32 & 9.38 & 0.19 & 0.06 & 0.77 \\
\hline IRAS $20286+1846$ & 9.44 & 9.45 & 1.58 & 0.63 & 2.26 & 8.93 & 8.86 & 0.41 & 0.20 & 0.91 \\
\hline IRAS $21077+3358$ & 9.86 & 9.85 & 2.88 & & 1.15 & 9.23 & 9.28 & 0.21 & & 0.40 \\
\hline IRAS $21272+2514$ & 9.66 & 9.60 & 1.97 & 0.15 & 1.73 & 9.11 & 8.97 & 0.34 & 0.15 & 0.56 \\
\hline IRAS $22055+3024$ & 9.22 & 9.27 & 0.28 & & 0.26 & 8.88 & 9.10 & 0.15 & & 0.22 \\
\hline IRAS $22116+0437$ & 10.27 & 9.85 & 3.16 & & 0.75 & 9.34 & 9.38 & 0.08 & & 0.40 \\
\hline IRAS $22491-1808$ & 9.47 & 9.33 & 1.38 & 0.45 & 0.98 & 9.07 & 8.94 & 0.43 & 0.45 & 0.57 \\
\hline IRAS $23028+0725$ & $\ldots$ & $\ldots$ & $\ldots$ & $\ldots$ & $\ldots$ & $\ldots$ & $\ldots$ & $\ldots$ & $\ldots$ & $\ldots$ \\
\hline IRAS $23233+0946$ & 9.68 & 9.59 & 1.74 & 0.36 & 1.45 & 9.30 & 9.17 & 0.47 & 0.36 & 0.76 \\
\hline IRAS $23365+3604$ & 9.80 & 9.63 & 2.37 & 0.27 & 0.92 & 9.31 & 9.18 & 0.35 & 0.27 & 0.43 \\
\hline IRAS 00163-1039 & 9.30 & 9.16 & 3.32 & & 0.95 & 9.00 & 8.83 & 0.52 & & 0.43 \\
\hline IRAS $01572+0009$ & 9.86 & 9.47 & 0.10 & & 0.04 & 9.60 & 9.55 & 0.07 & & 0.06 \\
\hline IRAS $05083+7936$ & 9.93 & 9.95 & 4.45 & & 2.10 & 9.68 & 9.57 & 0.62 & & 0.66 \\
\hline IRAS $06538+4628$ & 8.75 & 8.77 & 1.00 & & 0.60 & 8.51 & 8.50 & 0.45 & & 0.36 \\
\hline IRAS $08559+1053$ & 9.89 & 9.86 & 0.31 & 0.19 & 0.55 & 9.63 & 9.52 & 0.21 & 0.19 & 0.29 \\
\hline IRAS 09437+0317 & 9.10 & 9.16 & 1.47 & & 1.99 & 8.89 & 8.82 & 0.62 & & 0.77 \\
\hline IRAS $10565+2448$ & 9.90 & 9.80 & 1.53 & & 1.43 & 9.55 & 9.31 & 0.51 & & 0.51 \\
\hline IRAS 11119+3257 & 9.92 & 9.68 & 0.04 & 0.05 & 0.04 & 9.93 & 8.36 & 0.06 & 0.05 & 0.003 \\
\hline IRAS $13349+2438$ & - & - & - & - & - & $<9.39$ & $<9.10$ & $<0.01$ & & $<0.01$ \\
\hline IRAS $15001+1433$ & 9.86 & 9.86 & 0.30 & 0.14 & 0.43 & 9.54 & 9.44 & 0.16 & 0.14 & 0.20 \\
\hline IRAS $15206+3342$ & 9.81 & 9.75 & 0.38 & & 0.41 & 9.56 & 9.47 & 0.25 & & 0.27 \\
\hline IRAS $20460+1925$ & $\ldots$ & $\ldots$ & $\ldots$ & $\ldots$ & $\ldots$ & $\ldots$ & $\ldots$ & $\ldots$ & $\ldots$ & $\ldots$ \\
\hline
\end{tabular}


Table 6

(Continued)

\begin{tabular}{|c|c|c|c|c|c|c|c|c|c|c|}
\hline \multirow[t]{2}{*}{ Object } & \multicolumn{2}{|c|}{ PAHFIT Luminosity } & \multicolumn{3}{|c|}{ PAHFIT EW } & \multicolumn{2}{|c|}{ Spline-fit Luminosity } & \multicolumn{3}{|c|}{ Spline-fit EW } \\
\hline & $\begin{array}{c}6.2 \\
\left(\log L / L_{\odot}\right) \\
\end{array}$ & $\begin{array}{c}11.3 \\
\left(\log L / L_{\odot}\right) \\
\end{array}$ & $\begin{array}{c}6.2 \\
(\mu \mathrm{m})\end{array}$ & $\begin{array}{c}6.2 \text { ice } \\
(\mu \mathrm{m})\end{array}$ & $\begin{array}{l}11.3 \\
(\mu \mathrm{m})\end{array}$ & $\begin{array}{c}6.2 \\
\left(\log L / L_{\odot}\right) \\
\end{array}$ & $\begin{array}{c}11.3 \\
\left(\log L / L_{\odot}\right) \\
\end{array}$ & $\begin{array}{c}6.2 \\
(\mu \mathrm{m})\end{array}$ & $\begin{array}{c}6.2 \text { ice } \\
(\mu \mathrm{m})\end{array}$ & $\begin{array}{l}11.3 \\
(\mu \mathrm{m}) \\
\end{array}$ \\
\hline IRAS $23007+0836$ & 9.23 & 9.19 & 0.33 & & 0.34 & 8.99 & 8.89 & 0.19 & & 0.20 \\
\hline IRAS 23394-0353 & 9.26 & 9.16 & 2.24 & & 2.47 & 8.94 & 8.72 & 0.53 & & 0.64 \\
\hline IRAS $23498+2423$ & 9.52 & 9.53 & 0.06 & & 0.13 & 9.25 & 9.05 & 0.04 & & 0.06 \\
\hline
\end{tabular}

Notes. The "ice" $6.2 \mu \mathrm{m}$ PAH columns use a continuum that is corrected for water ice absorption (where present) at $6 \mu \mathrm{m}$. The symbol "-" indicates that PAHFIT fit no significant flux for a particular dust component.
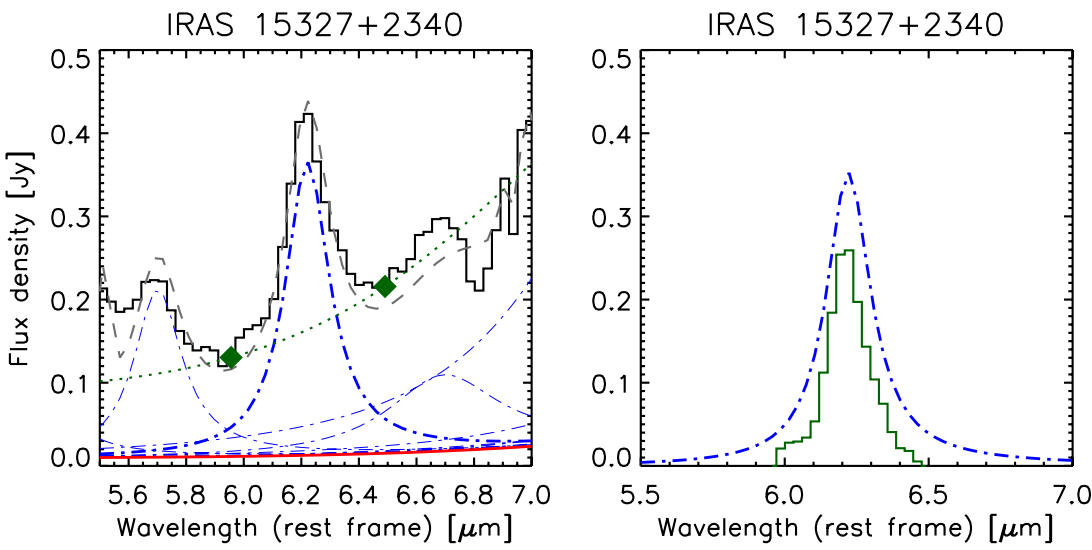

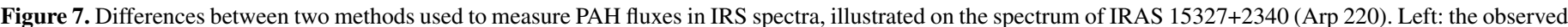

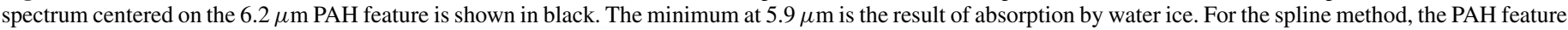

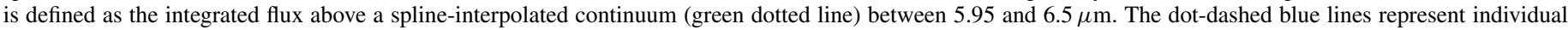

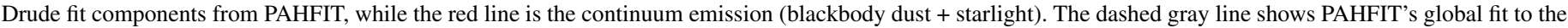

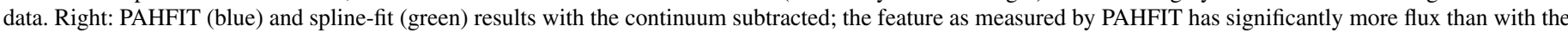
spline continuum, typical of nearly all galaxies in our sample.

showed absorption strong enough to affect the measured EW; the spline fit with the new continuum decreased the EW for all objects except IRAS $11180+1623$, for which the continuum levels as measured by the PAH fit and the silicate depth are nearly identical (within error). Since PAHFIT does not fit for ice absorption, we also calculate ice-corrected EW for objects showing absorption at $6 \mu \mathrm{m}$ by using the flux from PAHFIT and the inferred continuum from silicate measurements.

The $11.3 \mu \mathrm{m}$ PAH is seated atop the edge of the deep silicate absorption at $9.7 \mu \mathrm{m}$; determining an extinction-corrected continuum level for EW measurements is thus also difficult. Spectral mapping of AGN galaxies with ISOCAM has shown that $\mathrm{PAH}$ emission can be spatially extended and suppressed near the nucleus (Le Floc'h et al. 2001; Díaz-Santos et al. 2010); this means that the PAH emission in AGNs may be largely unaffected by dust absorption. Starburst galaxies, however, can show strong PAH emission in both the 6.2 and $11.3 \mu \mathrm{m}$ bands in the nuclear regions where the silicate optical depth is at its highest (Galliano et al. 2008), and is likely to affect continuum levels for PAH features. The measured $11.3 \mu \mathrm{m}$ PAH data using the spline-fit method are therefore likely to underestimate the luminosities.

\subsection{Absorption Features}

\subsubsection{Silicates}

The LR spectra show near-ubiquitous absorption from amorphous silicate dust, with a strong feature caused by a $\mathrm{Si}-\mathrm{O}$ stretching mode near $9.7 \mu \mathrm{m}$ and a weaker feature caused by an Si-O-Si bending mode near $18 \mu \mathrm{m}$ (Knacke \& Thomson 1973).
The presence of dust is unsurprising, as the characteristic extreme IR luminosities of ULIRGs are caused by large amounts of heated dust being thermally re-radiated. Hao et al. (2007) found that ULIRGs nearly uniformly show absorption in the two silicate features, in contrast to QSOs and some Seyfert galaxies which typically show the feature in emission (Siebenmorgen et al. 2005; Hao et al. 2005; Sturm et al. 2005; Schweitzer et al. 2008).

We measure the strength of the silicate absorption at both 9.7 and $18 \mu \mathrm{m}$ using the method of Spoon et al. (2007):

$$
S_{\lambda}=\ln \left(\frac{f_{\lambda}}{f_{\text {cont }}}\right),
$$

where $f_{\lambda}$ is the measured flux and $f_{\text {cont }}$ is the interpolated continuum at the feature extremum. More negative values of $S_{\text {sil }}$ represent deeper absorption. The expected continuum is calculated using a combination of spline and power-law fits, depending on the strength of the PAH and water ice features in the spectrum (Spoon et al. 2007).

All OHMs and $~ 95 \%$ of the non-masing galaxies showed absorption at both 9.7 and $18 \mu \mathrm{m}$ (Table 7); the average depth for the OHMs is $S_{9.7}=-1.8 \pm 0.8$, while the average depth of the non-masing galaxies is $S_{9.7}=-0.6 \pm 0.4$. The deepest absorption is in the OHM IRAS 04454-0838 $\left(S_{9.7}=-3.7\right)$, while only one object (the non-masing galaxy IRAS $13349+2438$ ) shows emission in both amorphous silicate features.

In addition to the amorphous silicate, we detect weaker features from crystalline silicate absorption in 19 OHMs, including bands at $11,16,19,23$, and $28 \mu \mathrm{m}$ (see Figure 8 
Table 7

Solid-phase Absorption Features

\begin{tabular}{|c|c|c|c|c|c|c|c|c|c|}
\hline \multirow[t]{2}{*}{ Object } & \multirow{2}{*}{$\begin{array}{c}6.0 \mu \mathrm{m} \mathrm{H}_{2} \mathrm{O} \text { Ice } \\
\tau\end{array}$} & \multicolumn{2}{|c|}{$6.85 \mu \mathrm{m}$ HAC } & \multicolumn{2}{|c|}{$7.25 \mu \mathrm{m}$ HAC } & \multirow{2}{*}{$\begin{array}{c}9.7 \mu \mathrm{m} \\
S_{\mathrm{sil}} \\
\end{array}$} & \multirow{2}{*}{$\begin{array}{c}18 \mu \mathrm{m} \\
S_{\mathrm{sil}}\end{array}$} & \multirow{2}{*}{$\begin{array}{c}16 \mu \mathrm{m} \\
S_{\text {sil }}^{\text {resid }}\end{array}$} & \multirow{2}{*}{$\begin{array}{c}23 \mu \mathrm{m} \\
S_{\text {sil }}^{\text {resid }} \\
\end{array}$} \\
\hline & & $\tau$ & Flux & $\tau$ & Flux & & & & \\
\hline IRAS $01355-1814$ & & & & & & -2.4 & -0.9 & -0.3 & -0.2 \\
\hline IRAS 01418+1651 & & 0.49 & -8.3 & & & -1.3 & -0.4 & & \\
\hline IRAS $01562+2528$ & & & & & & -0.7 & -0.3 & & \\
\hline IRAS 02524+2046 & & 0.20 & -0.4 & & & -0.9 & -0.3 & & \\
\hline IRAS $03521+0028$ & 0.42 & 0.13 & -0.4 & & & -1.4 & -0.2 & -0.1 & -0.1 \\
\hline IRAS $04121+0223$ & 1.61 & & & & & -1.0 & -0.2 & & \\
\hline IRAS $04454-4838$ & 0.42 & 0.35 & -9.0 & 0.10 & -1.5 & -3.7 & -1.0 & -0.3 & -0.2 \\
\hline IRAS 06487+2208 & & 0.23 & -4.6 & & & -1.2 & -0.3 & & \\
\hline IRAS $07163+0817$ & & & & & & -1.2 & -0.1 & & \\
\hline IRAS $07572+0533$ & & & & & & -0.6 & -0.3 & & \\
\hline IRAS 08201+2801 & 1.06 & 0.37 & -4.0 & 0.22 & -1.5 & -2.2 & -0.6 & -0.2 & -0.1 \\
\hline IRAS $08449+2332$ & & & & & & -1.2 & -0.5 & -0.1 & -0.1 \\
\hline IRAS $08474+1813$ & & 0.36 & -0.2 & & & -1.9 & -1.2 & & \\
\hline IRAS 09039+0503 & 0.98 & 0.15 & -0.5 & & & -2.0 & -0.6 & & \\
\hline IRAS 09539+0857 & & 0.24 & -2.5 & & & -3.1 & -1.2 & -0.4 & -0.2 \\
\hline IRAS $10035+2740$ & & & & & & -1.5 & -0.8 & & \\
\hline IRAS 10039-3338 & 0.23 & 0.23 & -166.3 & & & -3.1 & -1.0 & -0.4 & -0.3 \\
\hline IRAS $10173+0828$ & & & & & & -1.9 & -0.8 & -0.3 & -0.2 \\
\hline IRAS $10339+1548$ & & & & & & -1.1 & -0.05 & & \\
\hline IRAS $10378+1109$ & 0.72 & 0.18 & -0.6 & & & -2.0 & -0.3 & & \\
\hline IRAS $10485-1447$ & & 0.25 & -0.4 & & & -2.9 & -0.9 & & \\
\hline IRAS $11028+3130$ & & & & & & -2.6 & -1.0 & & \\
\hline IRAS $11180+1623$ & 0.54 & & & & & -1.7 & -0.5 & & \\
\hline IRAS $11524+1058$ & & 0.27 & -1.5 & $0.2:$ & $-0.3:$ & -1.5 & -0.8 & & \\
\hline IRAS 12018+1941 & & 0.16 & -2.6 & & & -1.4 & -0.4 & & \\
\hline IRAS 12032+1707 & 0.71 & 0.55 & -6.6 & 0.30 & -3.9 & -2.7 & -0.8 & & \\
\hline IRAS 12112+0305 & 0.59 & 0.41 & -3.5 & & & -1.8 & -0.3 & -0.2 & -0.1 \\
\hline IRAS $12540+5708$ & & & & & & -0.7 & -0.2 & & \\
\hline IRAS $13218+0552$ & & & & & & -0.5 & -0.4 & & \\
\hline IRAS $13428+5608$ & 0.50 & 0.40 & -28.5 & & & -2.0 & -0.5 & & \\
\hline IRAS $13451+1232$ & & & & & & -0.5 & -0.1 & & \\
\hline IRAS 14059+2000 & & & & & & -0.8 & -0.1 & & \\
\hline IRAS $14070+0525$ & 0.90 & 0.24 & -1.8 & 0.15 & -1.8 & -2.7 & -0.9 & & \\
\hline IRAS $14553+1245$ & & & & & & -1.3 & -0.5 & & \\
\hline IRAS $15327+2340$ & 0.68 & 0.35 & -50.1 & & & -3.1 & -0.4 & -0.2 & -0.1 \\
\hline IRAS 16090-0139 & 0.56 & 0.45 & -10.5 & 0.24 & -5.6 & -2.4 & -0.6 & & \\
\hline IRAS 16255+2801 & 0.54 & & & & & -2.2 & -0.6 & & \\
\hline IRAS $16300+1558$ & 0.61 & 0.38 & -2.2 & 0.21 & -1.1 & -2.7 & -0.7 & -0.3 & -0.1 \\
\hline IRAS 17207-0014 & 0.31 & 0.23 & -17.4 & & & -1.9 & -0.6 & -0.2 & -0.1 \\
\hline IRAS $18368+3549$ & & & & & & -1.8 & -0.2 & -0.3 & -0.2 \\
\hline IRAS $18588+3517$ & 0.72 & & & & & -2.2 & -0.6 & -0.3 & - \\
\hline IRAS 20100-4156 & 1.45 & 0.23 & -2.9 & 0.23 & -4.5 & -2.4 & -0.7 & -0.2 & -0.1 \\
\hline IRAS $20286+1846$ & 1.08 & & & & & -1.6 & -0.6 & & \\
\hline IRAS $21077+3358$ & & & & & & -1.9 & -0.7 & & \\
\hline IRAS $21272+2514$ & 1.66 & 0.21 & -0.7 & & & -2.8 & -0.7 & & \\
\hline IRAS $22055+3024$ & & & & & & -1.3 & -0.3 & & \\
\hline IRAS $22116+0437$ & & 0.04: & $-0.1:$ & & & -2.6 & -0.9 & -0.2 & -0.2 \\
\hline IRAS $22491-1808$ & 0.43 & 0.19 & -0.2 & & & -1.5 & -0.5 & -0.2 & - \\
\hline IRAS $23028+0725$ & $\ldots$ & $\ldots$ & $\ldots$ & $\ldots$ & $\ldots$ & $\ldots$ & -0.6 & & \\
\hline IRAS 23233+0946 & 0.44 & & & & & -1.9 & -0.4 & & \\
\hline IRAS $23365+3604$ & 0.66 & & & & & -2.0 & -0.5 & & \\
\hline IRAS 00163-1039 & & & & & & -0.5 & -0.1 & & \\
\hline IRAS 01572+0009 & & & & & & -0.2 & -0.2 & & \\
\hline IRAS $05083+7936$ & & & & & & -1.1 & -0.3 & & \\
\hline IRAS $06538+4628$ & & & & & & -0.5 & -0.2 & & \\
\hline IRAS 08559+1053 & 0.18 & & & & & -0.6 & -0.2 & & \\
\hline IRAS $09437+0317$ & & & & & & -1.1 & -0.3 & & \\
\hline IRAS $10565+2448$ & & & & & & -1.2 & -0.3 & & \\
\hline IRAS 11119+3257 & 0.19 & & & & & -0.7 & -0.3 & -0.2 & - \\
\hline IRAS $13349+2438$ & & & & & & 0.1 & 0.07 & & \\
\hline IRAS $15001+1433$ & 0.30 & & & & & -0.9 & -0.4 & & \\
\hline IRAS $15206+3342$ & & & & & & -0.4 & -0.2 & & \\
\hline IRAS $20460+1925$ & $\ldots$ & $\ldots$ & $\ldots$ & $\ldots$ & $\ldots$ & $\ldots$ & -0.4 & & \\
\hline
\end{tabular}


Table 7

(Continued)

\begin{tabular}{|c|c|c|c|c|c|c|c|c|c|}
\hline \multirow[t]{2}{*}{ Object } & \multirow{2}{*}{$\begin{array}{c}6.0 \mu \mathrm{m} \mathrm{H}_{2} \mathrm{O} \text { Ice } \\
\tau\end{array}$} & \multicolumn{2}{|c|}{$6.85 \mu \mathrm{m}$ HAC } & \multicolumn{2}{|c|}{$7.25 \mu \mathrm{m} \mathrm{HAC}$} & \multirow{2}{*}{$\begin{array}{c}9.7 \mu \mathrm{m} \\
S_{\text {sil }} \\
\end{array}$} & \multirow{2}{*}{$\begin{array}{c}18 \mu \mathrm{m} \\
S_{\text {sil }} \\
\end{array}$} & \multirow{2}{*}{$\begin{array}{l}16 \mu \mathrm{m} \\
S_{\text {sil }}^{\text {resid }} \\
\end{array}$} & \multirow{2}{*}{$\begin{array}{l}23 \mu \mathrm{m} \\
S_{\text {sil }}^{\text {resid }} \\
\end{array}$} \\
\hline & & $\tau$ & Flux & $\tau$ & Flux & & & & \\
\hline IRAS $23007+0836$ & & & & & & -0.3 & -0.1 & & \\
\hline IRAS 23394-0353 & & & & & & -0.7 & -0.3 & & \\
\hline IRAS $23498+2423$ & & & & & & -0.6 & -0.3 & & \\
\hline
\end{tabular}

Notes. Silicate strength is defined in Equation (5); the 9.7 and $18 \mu \mathrm{m}$ features are depths for amorphous silicates, while the 16 and $23 \mu \mathrm{m}$ crystalline features are residual depths measured after the $18 \mu \mathrm{m}$ feature was subtracted. Fluxes are given in $10^{-21} \mathrm{~W} \mathrm{~cm}^{-2}$; objects marked with a colon ":" represent uncertain detections.

for an example). We use the method of Spoon et al. (2006) to subtract off both the dust continuum and amorphous component to measure the residual optical depth at 16 and $23 \mu \mathrm{m}$, typically the strongest crystalline features (Table 7). The deepest $S_{16}$ occurs for IRAS $10039-3338$, at -0.4 ; however, the $\mathrm{S} / \mathrm{N}$ ratio means we are only sensitive to an absorption limit of $S_{16} \simeq-0.1$ and $S_{18} \simeq-0.05$. Only one detection of crystalline silicates is made in a non-masing galaxy, in IRAS $11119+3257$. Since all detections of crystalline silicates in OHMs have $S_{9.7}<-1.4$, the lower total dust column in non-masing galaxies is a likely contributor to the detection rate.

\subsubsection{Aliphatic Hydrocarbons}

Absorption bands arising from hydrogenated amorphous carbon grains (HACs) can also be significant contributors to diffuse dust in the galactic interstellar medium (ISM; Chiar et al. 2000). Observations from the Infrared Space Observatory (ISO; Spoon et al. 2001, 2002) and Spitzer (Dartois et al. 2007; Dartois \& Muñoz-Caro 2007) have identified HAC absorption features due to bending modes at $6.85 \mu \mathrm{m}\left(\mathrm{CH}_{2} / \mathrm{CH}_{3}\right)$ and $7.25 \mu \mathrm{m}\left(\mathrm{CH}_{3}\right)$ in ULIRGs. These aliphatic features represent a counterpart to the aromatic hydrocarbons responsible for PAH emission and are an abundant component of the ISM in luminous galaxies.

We detect absorption from the $6.85 \mu \mathrm{m}$ HAC transition in 27/51 galaxies in the OHM sample (Figure 8), with zero detections in the non-masing sample. The accompanying $7.25 \mu \mathrm{m}$ feature is detected in eight of the galaxies in which the $6.85 \mu \mathrm{m}$ feature is seen. The strength of the HAC is measured using a spline fit with pivots at 5.2, 5.6, 7.8, 14.0, and $26.0 \mu \mathrm{m}$ to determine the local continuum in the $6-8 \mu \mathrm{m}$ region (Spoon et al. 2007) and integrate the total flux within the absorption feature (Table 7). The average optical depth of the $6.85 \mu \mathrm{m}$ feature is $\tau_{6.85}=0.23 \pm 0.11$, and the average depth of the $7.25 \mu \mathrm{m}$ feature is $\tau_{7.25}=0.20 \pm 0.06$. Many of the galaxies with no detection of HACs, however, have limits from noise that are consistent with the absorption depths measured in brighter galaxies.

\subsubsection{Ices}

Absorption from ices in a variety of molecular species (including $\mathrm{H}_{2} \mathrm{O}, \mathrm{CO}, \mathrm{CO}_{2}$, and $\mathrm{CH}_{4}$ ) has been detected in spectroscopy of IR-bright galaxies (Spoon et al. 2000, 2001; Sturm et al. 2000). The band from water ice absorption stretching from 6 to $8 \mu \mathrm{m}$ is prominent (Figure 8 ) and was detected in $\sim 10 \%$ of a sample of bright galaxies using ISO (Spoon et al. 2002) and IRS (Armus et al. 2004, 2007; Spoon et al. 2005).

We detected water ice absorption at $6 \mu \mathrm{m}$ in $24 \mathrm{OHMs}$ and three non-masing galaxies. We use the spline continuum from fitting the $9.7 \mu \mathrm{m}$ silicate feature as the local $5.5-7 \mu \mathrm{m}$ continuum in order to obtain an optical depth spectrum for the $6 \mu \mathrm{m}$ absorption complex. The resulting water ice optical depths
Table 8

Gas-phase Absorption Features

\begin{tabular}{|c|c|c|c|c|c|c|}
\hline \multirow[t]{2}{*}{ Object } & \multicolumn{2}{|c|}{$\begin{array}{c}13.7 \mu \mathrm{m} \\
\mathrm{C}_{2} \mathrm{H}_{2}\end{array}$} & \multicolumn{2}{|c|}{$\begin{array}{c}14.02 \mu \mathrm{m} \\
\mathrm{HCN}\end{array}$} & \multicolumn{2}{|c|}{$15.0 \mu \mathrm{m} \mathrm{CO}_{2}$} \\
\hline & $f_{\text {norm }}$ & Flux & $f_{\text {norm }}$ & Flux & $f_{\text {norm }}$ & Flux \\
\hline IRAS 10039-3338 & 0.93 & -0.9 & 0.86 & -4.0 & & \\
\hline IRAS 12018+1941 & 0.95 & -0.7 & 0.94 & -0.8 & & \\
\hline IRAS $12540+5708$ & 0.95 & -9.5 & & & & \\
\hline IRAS $13218+0552$ & 0.82 & -1.1 & & & & \\
\hline IRAS $13428+5608$ & 0.93 & -0.8 & & & & \\
\hline IRAS $14070+0525$ & 0.79 & -0.4 & & & & \\
\hline IRAS $15327+2340$ & 0.84 & -5.8 & 0.90 & -5.6 & 0.94 & -2.0 \\
\hline IRAS 16090-0139 & 0.86 & -1.1 & & & & \\
\hline IRAS 17207-0014 & 0.93 & -0.7 & & & & \\
\hline IRAS 20100-4156 & 0.82 & -1.4 & 0.84 & -1.1 & & \\
\hline IRAS $22491-1808$ & 0.92 & -0.7 & & & & \\
\hline
\end{tabular}

Notes. $f_{\text {norm }}$ gives the peak depth of absorption features plotted in normalized flux units. Fluxes are measured in $10^{-21} \mathrm{~W} \mathrm{~cm}^{-2}$.

are tabulated in Table 7. We note that contamination by $6.2 \mu \mathrm{m}$ $\mathrm{PAH}$ emission and absorption by other species than water ice (Spoon et al. 2005) may add confusion in properly measuring optical depths.

\subsection{4. $\mathrm{C}_{2} \mathrm{H}_{2}, \mathrm{HCN}$, and $\mathrm{CO}_{2}$}

Previous mid-IR surveys have also identified bands of molecular gas absorption in ULIRGs (Spoon et al. 2006; Armus et al. 2007; Lahuis et al. 2007), including the vibration-rotation bands of acetylene $\left(\mathrm{C}_{2} \mathrm{H}_{2} ; 13.7 \mu \mathrm{m}\right)$, hydrogen cyanide ( $\mathrm{HCN}$; $14.02 \mu \mathrm{m})$, and carbon dioxide $\left(\mathrm{CO}_{2} ; 15.0 \mu \mathrm{m}\right)$. Lahuis et al. (2007) reported the detection of both $\mathrm{C}_{2} \mathrm{H}_{2}$ and $\mathrm{HCN}$ in 15 (U)LIRG nuclei, with detections of $\mathrm{CO}_{2}$ in four objects. Eight of the objects in the Lahuis sample are OHMs in our sample; we confirm detections of $\mathrm{C}_{2} \mathrm{H}_{2}$ in all eight galaxies, in addition to the OHMs IRAS 10039-3338 and IRAS 12018+1941 (Figure 8). $\mathrm{HCN}$ is detected in only 4/10 archival galaxies, meaning that we cannot confirm the $\mathrm{HCN}$ detection of four galaxies; since the optical depth of $\mathrm{HCN}$ is typically much weaker than that of $\mathrm{C}_{2} \mathrm{H}_{2}$, however, it is possible that our lower detection rate is a result of improved $\mathrm{S} / \mathrm{N}$ in their reduction process. We also confirm the detection of $\mathrm{CO}_{2}$ in IRAS $15327+2340$ (Arp 220). No galaxies in the non-masing control sample showed absorption in any molecular band, nor did any of the OHMs observed in our dedicated program.

Since these gas-phase absorption features are actually a blend of multiple absorption lines, the peak optical depth measured is a function of the velocity resolution of the spectrograph. We therefore report the integrated flux and the peak depth in normalized flux units $\left(f_{\text {norm}}\right.$, where the spectrum has been divided by the adopted continuum; Spoon et al. 2004) in Table 8 . 


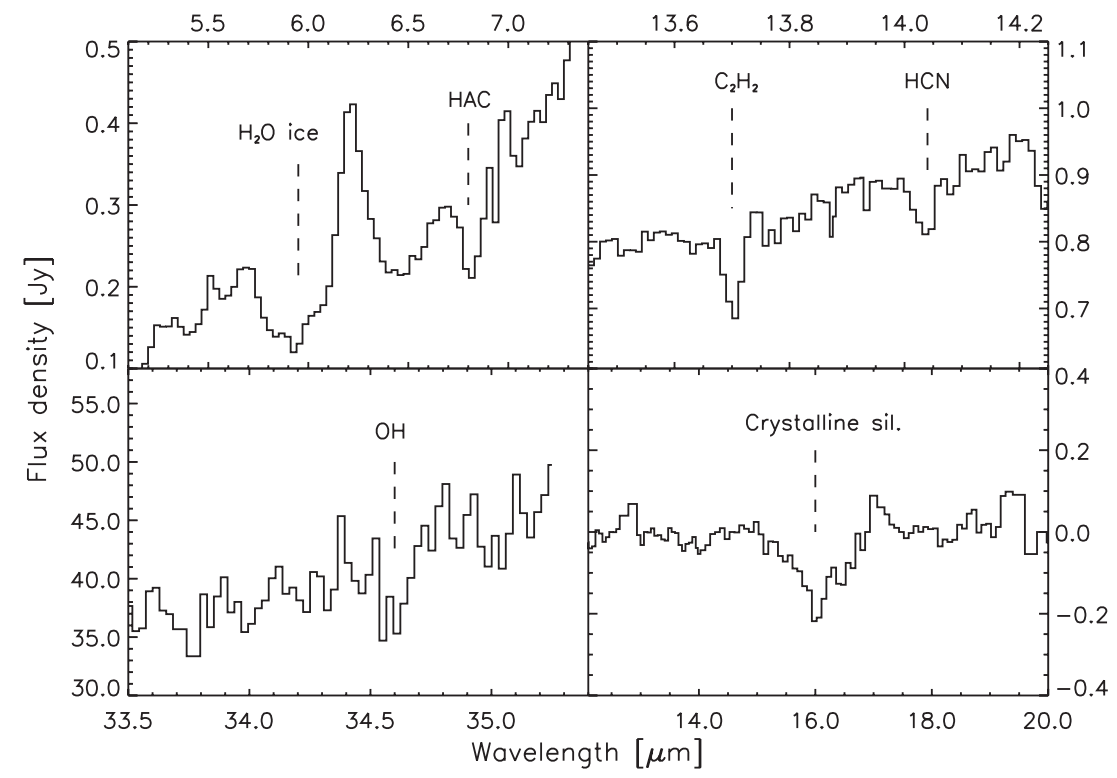

Figure 8. Examples of mid-IR absorption features for the OHM IRAS 15327+2340 (Arp 220). Top left: the $6.0 \mu \mathrm{m} \mathrm{H}_{2} \mathrm{O}$ ice and $6.85 \mu \mathrm{m}$ HAC absorption features. Top right: gas-phase $\mathrm{C}_{2} \mathrm{H}_{2} 13.7 \mu \mathrm{m}$ and $\mathrm{HCN} 14.0 \mu \mathrm{m}$ absorption. Bottom left: gas-phase $\mathrm{OH} 34.6 \mu \mathrm{m}$ absorption. Bottom right: residual optical depth of the $16 \mu \mathrm{m}$ crystalline silicate feature. Measurements for all labeled features are in Tables 7 and 8.

Lahuis et al. (2007) model abundances for ULIRGs with detections of $\mathrm{C}_{2} \mathrm{H}_{2}, \mathrm{HCN}$, and $\mathrm{CO}_{2}$, and suggest that they are associated with a phase of deeply embedded star formation, excluding the possibility of the features arising from an X-ray dominated region (XDR) powered by AGNs. Darling (2007) has also shown that OHMs have the highest mean molecular gas densities among starburst galaxies (traced by the $J=$ $1 \rightarrow 0$ rotational $\mathrm{HCN}$ transition) and also possess high dense molecular gas fractions, comprising a distinct population in the IR-CO relation. The results of Lahuis et al. (2007) show that 9/15 ULIRGs with absorption in both $\mathrm{C}_{2} \mathrm{H}_{2}$ and $\mathrm{HCN}$ are known OHMs in our Spitzer sample; this dense gas fraction $(\sim 50 \%)$ is nearly identical to the observed OHM fraction in starbursts with dense (ULIRGs with $L_{\mathrm{HCN}} / L_{\mathrm{CO}}>0.07$ ) fractions of molecular gas (Gao \& Solomon 2004b; Darling 2007; Baan et al. 2008).

Given that the $\mathrm{S} / \mathrm{N}$ ratio for the $\mathrm{OHM}$ and non-masing galaxies are of comparable magnitude, the lack of detection of any gas-phase species in the non-masing galaxies is a striking difference compared to the OHMs. Figure 9 shows the median stack of both samples near the regions of gas-phase absorption; while the $\mathrm{C}_{2} \mathrm{H}_{2}$ feature at $13.7 \mu \mathrm{m}$ can be clearly seen in the median OHM spectrum, neither the $\mathrm{HCN}$ nor the $\mathrm{CO}_{2}$ transition is prominent. Since the data have been median stacked (as opposed to a mean, which can be dominated by a few deep absorbers), this suggests low levels of $\mathrm{C}_{2} \mathrm{H}_{2}$ present in a significant fraction of the OHM host galaxies. No molecular absorption appears in the medianed spectrum for the non-masing galaxies.

While a connection between OHMs and dense molecular gas is known to exist, the lack of detected molecular absorption in the mid-IR for the majority of OHMs is not entirely unexpected. OHMs occur in merging galaxies where different populations of gas may be kinematically and thermally distinct, yet are observed as a single unresolved region within the Spitzer beam. Lahuis et al. (2007) suggest that high abundances of warm, dense gas are associated with deeply embedded star formation, where H II regions are prevented from expanding by large pressure gradients and extend the lifetime of the star formation process.

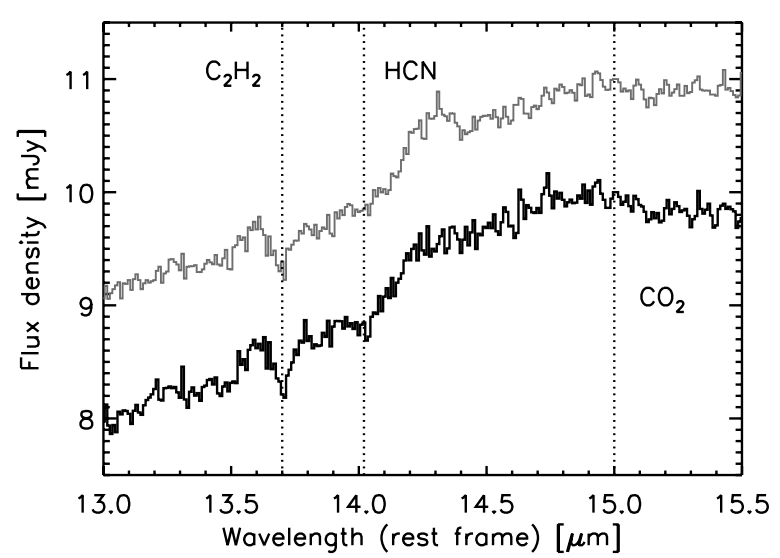

Figure 9. Medianed HR spectra for both OHMs (black) and non-masing (gray) galaxies, sampled at intervals of $0.01 \mu \mathrm{m}$ and normalized in flux at $15 \mu \mathrm{m}$. PAH emission is visible in bands centered at 13.6 and $14.2 \mu \mathrm{m}$. The dotted lines mark locations of gas-phase absorption in $\mathrm{C}_{2} \mathrm{H}_{2}(13.7 \mu \mathrm{m}), \mathrm{HCN}(14.02 \mu \mathrm{m})$, and $\mathrm{CO}_{2}(15.0 \mu \mathrm{m})$. The spectra are vertically offset to highlight the differences between the samples.

Baan et al. (2008) interpret dense gas abundances as excluding very hard radiation fields (such as those found in XDRs) that dissociate the molecules; they suggest that the molecular emission arises from PDRs surrounding $\mathrm{H}$ II regions. Although few OHMs show absorption from dense molecular gas, the OHM sample also has few identified AGNs or XDRs (only $4 / 51$ OHMs show [Ne v] at $14 \mu \mathrm{m}$ ). The connection between dense molecular gas and the presence of an AGN is thus unclear based on this data alone.

\subsubsection{Gas-phase $\mathrm{OH}$}

For three OHMs, we report detection of the ${ }^{2} \Pi_{1 / 2} J=5 / 2 \rightarrow$ ${ }^{2} \Pi_{3 / 2} J=3 / 2 \mathrm{OH}$ absorption doublet near $34.616 \mu \mathrm{m}$ : III Zw 35 (IRAS 01418+1651), Mrk 273 (IRAS 13428+5608), and Arp 220 (IRAS $15327+2340$ ). This feature is generally difficult to detect since it lies near the noisy, far-red edge of the LH module; for all objects with $z>0.08$, it is redshifted out of the IRS range. $\mathrm{OH}$ absorption at $34.6 \mu \mathrm{m}$ in Arp 220 was first 


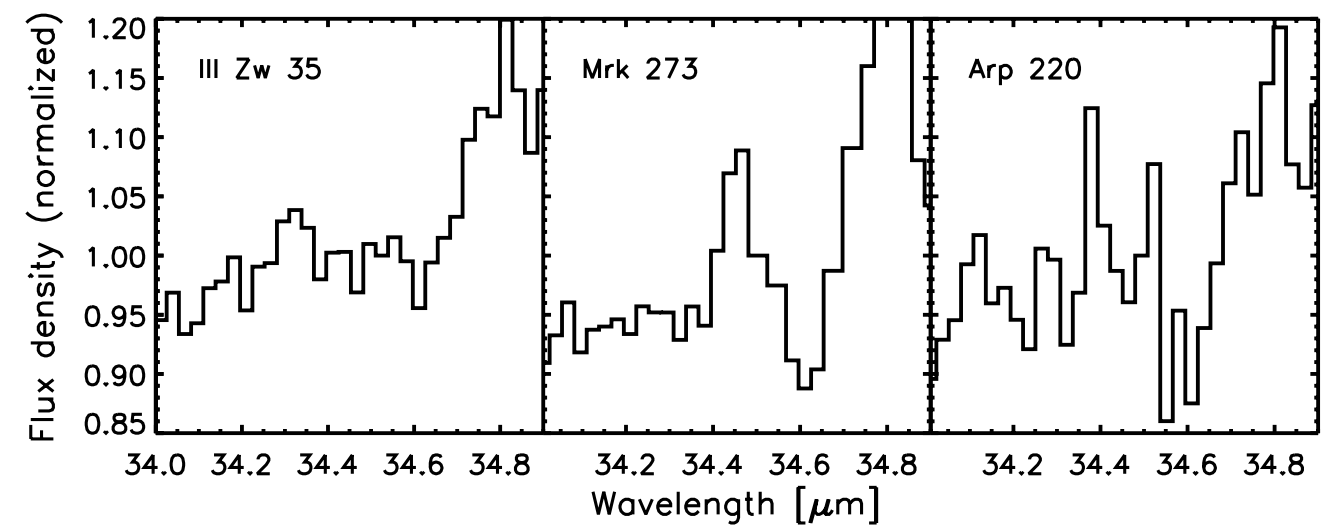

Figure 10. $34.6 \mu \mathrm{m} \mathrm{OH}$ absorption feature in III Zw 35, Mrk 273, and Arp 220. All spectra are been normalized in flux near $34.5 \mu \mathrm{m}$.

Table 9

Properties of OH Gas-phase Absorption

\begin{tabular}{|c|c|c|c|c|c|c|c|}
\hline $\mathrm{OHM}$ & $\begin{array}{c}f_{\mathrm{OH}} \\
\left(10^{-21} \mathrm{~W} \mathrm{~cm}^{-2}\right)\end{array}$ & $\tau_{\text {peak }}$ & $\begin{array}{c}\text { EW } \\
\left(10^{-3} \mu \mathrm{m}\right)\end{array}$ & $\begin{array}{c}N_{\mathrm{OH}} \\
\left(\mathrm{cm}^{-2}\right)\end{array}$ & $\begin{array}{c}\gamma_{\mathrm{abs}} \\
\left.\text { (photons s }^{-1}\right)\end{array}$ & 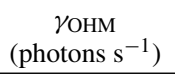 & $\begin{array}{c}\phi_{\text {pump }} \\
(\%) \\
\end{array}$ \\
\hline IRAS 01418+1651 (III Zw 35) & -6.1 & 0.15 & 5.5 & $1.1 \times 10^{17}$ & $1.7 \times 10^{54}$ & $1.8 \times 10^{53}$ & 10 \\
\hline IRAS $13428+5608$ (Mrk 273) & -28.5 & 0.14 & 10.2 & $2.1 \times 10^{17}$ & $1.7 \times 10^{55}$ & $1.4 \times 10^{53}$ & 0.8 \\
\hline IRAS $15327+2340($ Arp 220) & -172 & 0.21 & 15.0 & $3.0 \times 10^{17}$ & $2.3 \times 10^{55}$ & $1.6 \times 10^{53}$ & 0.7 \\
\hline
\end{tabular}

Note. The pumping efficiency $\left(\phi_{\text {pump }}=\gamma_{\mathrm{OHM}} / \gamma_{\mathrm{abs}} \times 100\right)$ assumes all pumping comes from the $34.6 \mu \mathrm{m}$ transition.

reported by Skinner et al. (1997) using ISO and confirmed with the IRS by Farrah et al. (2007), who incorrectly identified it as the $\mathrm{OH}^{-}$ion. All $\mathrm{OH}$ absorption features are well fit with a single Gaussian (Figure 10), since the separation between the doublet features $(\Delta \lambda \simeq 0.02 \mu \mathrm{m})$ is comparable to the resolution element in the LH module. No detection of $\mathrm{OH}$ absorption was made for any of the non-masing galaxies.

Assuming the $\mathrm{OH}$ transitions are optically thin, we can use the EW to derive a column density for the $\mathrm{OH}$ ground state, which is likely to be a good proxy for the total column at typical molecular cloud densities (Bradford et al. 1999):

$$
N_{l}=\frac{E W}{A_{u l}} \frac{8 \pi c}{\lambda^{4}} \frac{g_{l}}{g_{u}} .
$$

$\mathrm{OH}$ column densities for all galaxies are quite similar, lying between (1-3) $\times 10^{17} \mathrm{~cm}^{-2}$ (Table 9). The measured $N_{\mathrm{OH}}$ from the IRS data for Arp 220 also agrees within a factor of two of the column measured with ISO (Skinner et al. 1997). Limits for galaxies in which the $34.6 \mu \mathrm{m} \mathrm{OH}$ feature is not detected are of order $N_{\mathrm{OH}} \lesssim 1 \times 10^{17} \mathrm{~cm}^{-2}$.

We compare the $N_{\mathrm{OH}}$ derived from the rotational $34.6 \mu \mathrm{m}$ transitions to the $\mathrm{OH}$ column density measured in galaxies who show the hyperfine $1667 \mathrm{MHz}$ feature in absorption. The majority of such galaxies are ULIRGs of comparable luminosity to the galaxies in our non-masing sample. Measurements from $10 \mathrm{OH}$ absorbers (Baan et al. 1992; Darling 2007) give $N_{\mathrm{OH}}=T_{\mathrm{ex}}(1.8 \pm 1.9) \times 10^{15} \mathrm{~cm}^{-2}$, where $T_{\mathrm{ex}}$ is the $\mathrm{OH}$ excitation temperature in $\mathrm{K}$. If the dust and gas are well mixed, then the temperature of the dust $(\sim 50-100 \mathrm{~K})$ can be used as a proxy for $T_{\mathrm{ex}}$. This gives $\mathrm{OH}$ column densities for both OHMs $(34.6 \mu \mathrm{m})$ and non-masing galaxies $(1667 \mathrm{MHz})$ with comparable values of $N_{\mathrm{OH}} \simeq 10^{17} \mathrm{~cm}^{-2}$. If so, then this addresses one of the crucial differences between OHMs and non-masing ULIRGs - namely, that differences in the abundance of masing molecules are not a key factor for triggering an OHM.
The amount of $\mathrm{OH}$ available in the galaxy can also test models of the OHM pumping mechanism. Skinner et al. (1997) computed the photon flux $\left(\gamma_{\mathrm{abs}}=L_{\mathrm{abs}}^{\mathrm{OH}} / h v_{\mathrm{OH}}\right)$ absorbed in the $34.6 \mu \mathrm{m}$ transition from the OHM Arp 220. They found that $\gamma_{\mathrm{abs}}$ is roughly $1 \%$ of the photon flux in the OHM $\left(\gamma_{\mathrm{OHM}}=L_{\mathrm{OHM}} / h v_{\mathrm{OHM}}\right)$. If the $18 \mathrm{~cm}$ and mid-IR pumping photons lie along the same line of sight, this means that pumping photons from the $34.6 \mu \mathrm{m}$ transition alone can power the OHM (given an efficiency of $\sim 1 \%$ or higher). While radiative transfer models from Lockett \& Elitzur (2008) suggest that the $53 \mu \mathrm{m}$ $\mathrm{OH}$ transition likely contributes more pumping photons than the $34.6 \mu \mathrm{m}$ line, the energetics are consistent with the basic accepted mid-IR pumping model.

For the galaxies in our sample with $\mathrm{OH}$ absorption, Arp 220 and Mrk 273 would require pumping efficiencies on the order of $1 \%$ to power the OHMs from the $34.6 \mu \mathrm{m}$ transition alone. III $\mathrm{Zw} 35$ shows the weakest $\mathrm{OH}$ absorption among our detections and would require an efficiency of $\phi_{\text {pump }} \simeq 10 \%$.

\section{CONCLUSIONS}

We present mid-infrared spectra and photometry for $51 \mathrm{OH}$ megamasers taken with the IRS on Spitzer, along with 15 galaxies confirmed to have no megamaser emission above $L_{\mathrm{OH}}=10^{2.3} L_{\odot}$. All objects in both samples have full coverage in both the LR and HR IRS modules. We measure both emission (PAH, $\mathrm{H}_{2}$, and fine-structure atomic transitions) and absorption (silicates, HAC grains, and molecular bands) features, with full spectra, line fluxes, EWs, and absorption depths presented for each object.

The majority of the galaxies closely resemble standard mid-IR ULIRG templates, with the low-resolution emission dominated by moderate-to-deep amorphous silicate absorption at 9.7 and $18 \mu \mathrm{m}$ and PAH features at 6.2, 7.7, 8.6, 11.3 and $12.7 \mu \mathrm{m}$. The OHMs (on average) show deeper silicate absorption and steeper continuum slopes than the non-masing galaxies. Crystalline silicate absorption is detected in roughly a third of OHMs, but in 
Table 10

Hi-res Line Fluxes for Common Atomic Emission Lines with HR Sky Subtraction

\begin{tabular}{|c|c|c|c|c|}
\hline Object & $\begin{array}{c}\text { [S IV] } \\
10.511 \mu \mathrm{m} \\
\end{array}$ & $\begin{array}{c}{[\mathrm{Ne} \mathrm{II}]} \\
12.814 \mu \mathrm{m}\end{array}$ & $\begin{array}{c}{[\mathrm{Ne} \text { III] }} \\
15.555 \mu \mathrm{m} \\
\end{array}$ & $\begin{array}{c}{[\mathrm{S} \mathrm{III}]} \\
18.713 \mu \mathrm{m} \\
\end{array}$ \\
\hline IRAS $01562+2528$ & $\ldots$ & $\ldots$ & $\ldots$ & 0.65 \\
\hline IRAS $02524+2046$ & $\ldots$ & $\ldots$ & $\ldots$ & 0.47 \\
\hline IRAS $04121+0223$ & $\ldots$ & $\ldots$ & $\ldots$ & 0.67 \\
\hline IRAS 04454-4838 & 0.42 & 1.95 & 0.43 & $\ldots$ \\
\hline IRAS 06487+2208 & $\ldots$ & $\ldots$ & $\ldots$ & 5.01 \\
\hline IRAS $07163+0817$ & $\ldots$ & $\ldots$ & $\ldots$ & 1.34 \\
\hline IRAS $08201+2801$ & $\ldots$ & $\cdots$ & $\ldots$ & 0.41 \\
\hline IRAS $08449+2332$ & $\ldots$ & $\ldots$ & $\ldots$ & 1.60 \\
\hline IRAS $08474+1813$ & $\ldots$ & $\ldots$ & $\ldots$ & 0.14 \\
\hline IRAS $10035+2740$ & $\ldots$ & $\ldots$ & $\ldots$ & 0.35 \\
\hline IRAS 10039-3338 & 0.98 & 17.22 & 4.20 & 8.08 \\
\hline IRAS $10339+1548$ & $\ldots$ & $\ldots$ & $\ldots$ & 0.97 \\
\hline IRAS $11180+1623$ & $\ldots$ & $\ldots$ & $\ldots$ & 0.40 \\
\hline IRAS $11524+1058$ & $\ldots$ & $\ldots$ & $\ldots$ & 0.27 \\
\hline IRAS $12540+5708$ & $\ldots$ & 19.47 & $\ldots$ & $\ldots$ \\
\hline IRAS $14059+2000$ & $\ldots$ & $\ldots$ & $\ldots$ & 0.59 \\
\hline IRAS $14553+1245$ & $\ldots$ & $\ldots$ & $\ldots$ & 1.58 \\
\hline IRAS $15327+2340$ & $\ldots$ & 59.39 & 6.73 & 7.54 \\
\hline IRAS $16255+2801$ & $\ldots$ & $\ldots$ & $\ldots$ & 1.54 \\
\hline IRAS $18368+3549$ & $\ldots$ & $\ldots$ & $\ldots$ & 1.01 \\
\hline IRAS $18588+3517$ & $\ldots$ & $\ldots$ & $\ldots$ & 2.89 \\
\hline IRAS $20286+1846$ & $\ldots$ & $\ldots$ & $\ldots$ & 0.48 \\
\hline IRAS $21077+3358$ & $\ldots$ & $\ldots$ & $\ldots$ & 0.74 \\
\hline IRAS $21272+2514$ & $\ldots$ & 2.22 & 0.33 & 0.40 \\
\hline IRAS $22055+3024$ & $\ldots$ & $\ldots$ & $\ldots$ & 1.16 \\
\hline IRAS $22116+0437$ & $\cdots$ & $\cdots$ & $\cdots$ & 0.90 \\
\hline IRAS 00163-1039 & 2.64 & 87.43 & 14.30 & 32.10 \\
\hline IRAS $05083+7936$ & $\ldots$ & 49.95 & 7.63 & 19.60 \\
\hline IRAS $06538+4628$ & 0.84 & 47.34 & 5.90 & 20.49 \\
\hline IRAS 09437+0317 & $\ldots$ & 8.72 & 1.22 & 3.58 \\
\hline IRAS 23394-0353 & $\ldots$ & 46.45 & 7.60 & 18.50 \\
\hline
\end{tabular}

Notes. Fluxes are in $10^{-21} \mathrm{~W} \mathrm{~cm}^{-2}$. No data are given for the $26 \mathrm{OHMs}$ in our program for lines with $\lambda_{\text {rest }} \lesssim 16 \mu \mathrm{m}$ since we have no SH sky subtraction available for these objects.

only 1 out of 15 non-masing galaxies. OHMs are also the only galaxies in our sample to show absorption from HACs, gas- phase $\mathrm{HCN}, \mathrm{C}_{2} \mathrm{H}_{2}$, and $\mathrm{CO}_{2}$; however, higher average noise in the non-masing spectra mean that features at similar absorption depths could be obscured due to lack of sensitivity. Highresolution spectra show emission from [Ne II] and [Ne III] in almost all galaxies, with emission from [S III], [S IV], and [O IV] commonly detected. The high-ionization [Ne v] line (a clear tracer of $\mathrm{AGN}$ ) is detected in $<10 \%$ of OHMs and in $53 \%$ of the non-masing galaxies. Almost all galaxies in both samples also show emission in multiple $\mathrm{H}_{2}$ rotational transitions.

We also measure the $34.6 \mu \mathrm{m} \mathrm{OH}$ transition in three OHMs. $\mathrm{OH}$ column densities derived from the mid-IR $\mathrm{OH}$ transition are of the same order of magnitude as the column densities derived from the $1667 \mathrm{MHz} \mathrm{OH}$ transition for ULIRGs in the literature. We interpret this as evidence that the $\mathrm{OH}$ abundances in both OHMs and non-masing galaxies are similar, and are not a limiting factor for megamaser emission.

A companion paper (Willett et al. 2011, Paper II) presents a full analysis of the mid-IR data, with comparisons between the two samples and connections to the OHM properties.

This work is based on observations made with the Spitzer Space Telescope, which is operated by the Jet Propulsion Laboratory, California Institute of Technology under a contract with NASA. Support for this work was provided by NASA through grant 30407 issued by JPL/Caltech. We have made extensive use of the NASA/IPAC Extragalactic Database (NED) which is operated by JPL and Caltech under contract with NASA. Many thanks are due to J.-D. Smith for help with PAHFIT, N. Halverson for comments on computing flux limits, D. Farrah for useful discussions, and to the Spitzer Science Center for hosting K.W.W. and J.D. in 2007 April. V.C. acknowledges partial support from the EU ToK grant 39965 and FP7-REGPOT 206469.

\section{APPENDIX \\ HIGH-RESOLUTION DATA WITH BACKGROUND SKY SUBTRACTION}

As discussed in Section 3, the reduction process for the overall sample is slightly different for some archival galaxies that did not have separate IRS sky backgrounds in the HR modules. Since

Table 11

Hi-res Line Fluxes for Rarer Atomic Emission Lines with HR Sky Subtraction

\begin{tabular}{|c|c|c|c|c|c|c|c|c|c|}
\hline $\begin{array}{c}\text { Object } \\
\lambda_{\text {rest }}(\mu \mathrm{m})\end{array}$ & $\begin{array}{l}\text { H I 7-6 } \\
12.368\end{array}$ & $\begin{array}{l}{[\mathrm{Ne} \mathrm{V}]} \\
14.322\end{array}$ & $\begin{array}{l}{[\mathrm{Cl} \mathrm{II}]} \\
14.369\end{array}$ & $\begin{array}{l}{[\mathrm{Fe} \mathrm{II}]} \\
17.936\end{array}$ & $\begin{array}{l}{[\mathrm{Ne} \mathrm{V}]} \\
24.318\end{array}$ & $\begin{array}{l}{[\mathrm{O} \text { IV }]} \\
25.890\end{array}$ & $\begin{array}{l}{[\mathrm{Fe} \mathrm{II}]} \\
25.988\end{array}$ & $\begin{array}{l}\text { [S III] } \\
33.481\end{array}$ & $\begin{array}{l}{[\mathrm{Si} \mathrm{II}]} \\
34.815\end{array}$ \\
\hline IRAS 04454-4838 & $\ldots$ & $\ldots$ & $\ldots$ & $\ldots$ & $\ldots$ & 1.12 & $\ldots$ & 0.90 & $\ldots$ \\
\hline IRAS 06487+2208 & $\ldots$ & $\ldots$ & $\ldots$ & $\ldots$ & $\ldots$ & 1.12 & $\ldots$ & $\ldots$ & $\ldots$ \\
\hline IRAS $07163+0817$ & $\ldots$ & $\ldots$ & $\ldots$ & 0.73 & $\ldots$ & $\ldots$ & $\ldots$ & $\ldots$ & $\ldots$ \\
\hline IRAS $10339+1548$ & $\ldots$ & $\ldots$ & $\ldots$ & $\ldots$ & 0.84 & 2.55 & $\ldots$ & $\ldots$ & $\ldots$ \\
\hline IRAS $11524+1058$ & $\ldots$ & $\ldots$ & $\ldots$ & 0.35 & $\ldots$ & 0.59 & $\ldots$ & $\ldots$ & $\ldots$ \\
\hline IRAS $14553+1245$ & $\ldots$ & $\ldots$ & $\ldots$ & 0.41 & $\ldots$ & $\ldots$ & $\ldots$ & $\ldots$ & $\ldots$ \\
\hline IRAS $16255+2801$ & $\ldots$ & $\ldots$ & $\ldots$ & $\ldots$ & $\ldots$ & 0.46 & $\ldots$ & $\ldots$ & $\ldots$ \\
\hline IRAS $18368+3549$ & $\ldots$ & $\ldots$ & $\ldots$ & 0.51 & $\ldots$ & $\ldots$ & $\ldots$ & $\ldots$ & $\ldots$ \\
\hline IRAS $21272+2514$ & 0.20 & 0.10 & $\ldots$ & $\ldots$ & $\ldots$ & $\ldots$ & $\ldots$ & $\ldots$ & $\ldots$ \\
\hline IRAS $00163-1039$ & 0.64 & & .. & $\ldots$ & $\ldots$ & 1.56 & 2.46 & 32.18 & 74.28 \\
\hline IRAS $05083+7936$ & $\ldots$ & 0.58 & 0.43 & $\ldots$ & $\ldots$ & 1.53 & 1.97 & 29.34 & 43.28 \\
\hline IRAS $06538+4628$ & $\ldots$ & 1.08 & $\ldots$ & 1.06 & $\ldots$ & $\ldots$ & 2.15 & 37.21 & 48.72 \\
\hline IRAS $09437+0317$ & $\ldots$ & $\ldots$ & $\ldots$ & $\ldots$ & $\ldots$ & 0.47 & 0.68 & 9.60 & 22.74 \\
\hline IRAS 23394-0353 & 0.59 & $\ldots$ & $\ldots$ & $\ldots$ & $\ldots$ & 1.49 & 2.53 & 42.37 & 53.56 \\
\hline
\end{tabular}

Notes. Fluxes are in $10^{-21} \mathrm{~W} \mathrm{~cm}^{-2}$. No data are given for the 26 OHMs in our program for lines with $\lambda_{\text {rest }} \lesssim 16 \mu \mathrm{m}$ since we have no SH sky subtraction available for these objects. 
Table 12

Hi-res Line Fluxes and Upper Limits for $\mathrm{H}_{2}$ Transitions with HR Sky Subtraction

\begin{tabular}{ccccc}
\hline Object & $\mathrm{H}_{2} S(3)$ & $\mathrm{H}_{2} S(2)$ & $\mathrm{H}_{2} S(1)$ & $\mathrm{H}_{2} S(0)$ \\
$\lambda_{\text {rest }}(\mu \mathrm{m})$ & 9.67 & 12.28 & 17.04 & 28.22 \\
\hline IRAS $01562+2528$ & $\ldots$ & $\ldots$ & 0.78 & $\ldots$ \\
IRAS $02524+2046$ & $\ldots$ & $\ldots$ & 0.72 & $\ldots$ \\
IRAS 04454-4838 & 1.05 & 1.21 & 3.03 & 0.76 \\
IRAS 06487+2208 & $\ldots$ & $\ldots$ & 2.03 & $\ldots$ \\
IRAS 08201+2801 & $\ldots$ & $\ldots$ & 0.51 & $\ldots$ \\
IRAS 08449+2332 & $\ldots$ & $\ldots$ & 1.40 & $\ldots$ \\
IRAS 08474+1813 & $\ldots$ & $\ldots$ & 0.27 & $\ldots$ \\
IRAS $10035+2740$ & $\ldots$ & $\ldots$ & 1.16 & $\ldots$ \\
IRAS $10039-3338$ & 3.39 & 1.79 & 3.85 & $\ldots$ \\
IRAS $10339+1548$ & $\ldots$ & $\ldots$ & 0.46 & $\ldots$ \\
IRAS $11180+1623$ & $\ldots$ & $\ldots$ & 0.89 & $\ldots$ \\
IRAS $11524+1058$ & $\ldots$ & $\ldots$ & 0.81 & $\ldots$ \\
IRAS $12540+5708$ & 2.42 & 4.22 & $\ldots$ & $\ldots$ \\
IRAS $14059+2000$ & $\ldots$ & $\ldots$ & 2.55 & $\ldots$ \\
IRAS $15327+2340$ & $\ldots$ & 7.36 & 15.42 & 14.55 \\
IRAS $16255+2801$ & $\ldots$ & $\ldots$ & 0.55 & $\ldots$ \\
IRAS 21077+3358 & $\ldots$ & $\ldots$ & 1.17 & $\ldots$ \\
IRAS 21272+2514 & 0.26 & 0.35 & 0.68 & $\ldots$ \\
IRAS 22116+0437 & $\ldots$ & $\ldots$ & 1.35 & $\ldots$ \\
IRAS 23028+0725 & $\ldots$ & $\ldots$ & 1.11 & $\ldots$ \\
\hline IRAS 00163-1039 & 2.18 & 2.82 & 6.01 & $\ldots$ \\
IRAS 05083+7936 & 2.34 & 2.64 & 5.11 & $\ldots .99$ \\
IRAS 06538+4628 & $\ldots$ & 3.33 & 8.70 & 1.78 \\
IRAS 09437+0317 & $\ldots$ & $\ldots$ & 2.67 & 1.38 \\
IRAS 23394-0353 & 3.40 & 2.25 & 4.99 & $\ldots$ \\
\hline
\end{tabular}

Notes. Fluxes are in $10^{-21} \mathrm{~W} \mathrm{~cm}^{-2}$. No data are given for the $26 \mathrm{OHMs}$ in our program for the $S(2)$ or $S(3)$ lines since we have no $\mathrm{SH}$ sky subtraction available for these objects.

much of our subsequent analysis (Paper II) depends on statistical comparisons between the two samples, we chose to minimize possible systematic errors and reduced all galaxies in a uniform manner without HR sky subtraction. These data are, however, likely to be a more reliable indicator of the absolute flux levels due to subtraction of the zodiacal background; therefore, we also present atomic and molecular line fluxes for these galaxies (Tables 10-12).

\section{REFERENCES}

Armus, L., et al. 2004, ApJS, 154, 178

Armus, L., et al. 2007, ApJ, 656, 148

Baan, W. A., Haschick, A., \& Henkel, C. 1992, AJ, 103, 728

Baan, W. A., Henkel, C., Loenen, A. F., Baudry, A., \& Wiklind, T. 2008, A\&A, 477,747

Baan, W. A., Wood, P. A. D., \& Haschick, A. D. 1982, ApJ, 260, L49

Bradford, C. M., et al. 1999, in The Universe as Seen by ISO, ed. P. Cox \& M. Kessler (ESA Special Publication, Vol. 427; Noordwijk: ESA), 861

Brandl, B. R., et al. 2006, ApJ, 653, 1129

Chiar, J. E., Tielens, A. G. G. M., Whittet, D. C. B., Schutte, W. A., Boogert, A. C. A., Lutz, D., van Dishoeck, E. F., \& Bernstein, M. P. 2000, ApJ, 537, 749

Condon, J. J., Cotton, W. D., Greisen, E. W., Yin, Q. F., Perley, R. A., Taylor, G. B., \& Broderick, J. J. 1998, AJ, 115, 1693

Darling, J. 2007, ApJ, 669, L9

Darling, J., \& Giovanelli, R. 2000, AJ, 119, 3003

Darling, J., \& Giovanelli, R. 2001, AJ, 121, 1278

Darling, J., \& Giovanelli, R. 2002a, AJ, 124, 100

Darling, J., \& Giovanelli, R. 2002b, ApJ, 572, 810
Dartois, E., \& Muñoz-Caro, G. M. 2007, A\&A, 476, 1235

Dartois, E., et al. 2007, A\&A, 463, 635

Desai, V., et al. 2007, ApJ, 669, 810

Díaz-Santos, T., et al. 2010, ApJ, 723, 993

Draine, B. T. 2003, ARA\&A, 41, 241

Farrah, D., et al. 2007, ApJ, 667, 149

Galliano, F., Madden, S. C., Tielens, A. G. G. M., Peeters, E., \& Jones, A. P. 2008, ApJ, 679, 310

Gao, Y., \& Solomon, P. M. 2004a, ApJS, 152, 63

Gao, Y., \& Solomon, P. M. 2004b, ApJ, 606, 271

Genzel, R., et al. 1998, ApJ, 498, 579

Hao, L., Weedman, D. W., Spoon, H. W. W., Marshall, J. A., Levenson, N. A., Elitzur, M., \& Houck, J. R. 2007, ApJ, 655, L77

Hao, L., et al. 2005, ApJ, 625, L75

Henkel, C., Guesten, R., \& Baan, W. A. 1987, A\&A, 185, 14

Henkel, C., \& Wilson, T. L. 1990, A\&A, 229, 431

Higdon, S. J. U., Armus, L., Higdon, J. L., Soifer, B. T., \& Spoon, H. W. W. 2006, ApJ, 648, 323

Higdon, S. J. U., et al. 2004, PASP, 116, 975

Hinshaw, G., et al. 2009, ApJS, 180, 225

Houck, J. R., et al. 2004, ApJS, 154, 18

Imanishi, M., Dudley, C. C., Maiolino, R., Maloney, P. R., Nakagawa, T., \& Risaliti, G. 2007, ApJS, 171, 72

Kent, B., Braatz, J., \& Darling, J. 2002, BAAS, 34, 1194

Knacke, R. F., \& Thomson, R. K. 1973, PASP, 85, 341

Lahuis, F., et al. 2007, ApJ, 659, 296

Le Floc'h, E., Mirabel, I. F., Laurent, O., Charmandaris, V., Gallais, P., Sauvage, M., Vigroux, L., \& Cesarsky, C. 2001, A\&A, 367, 487

Lockett, P., \& Elitzur, M. 2008, ApJ, 677, 985

Lutz, D., Spoon, H. W. W., Rigopoulou, D., Moorwood, A. F. M., \& Genzel, R. 1998, ApJ, 505, L103

Marshall, J. A., Herter, T. L., Armus, L., Charmandaris, V., Spoon, H. W. W., Bernard-Salas, J., \& Houck, J. R. 2007, ApJ, 670, 129

Peeters, E., Spoon, H. W. W., \& Tielens, A. G. G. M. 2004, ApJ, 613, 986

Rigopoulou, D., Kunze, D., Lutz, D., Genzel, R., \& Moorwood, A. F. M. 2002, A\&A, 389, 374

Roussel, H., et al. 2007, ApJ, 669, 959

Sanders, D. B., Mazzarella, J. M., Kim, D.-C., Surace, J. A., \& Soifer, B. T. 2003, AJ, 126, 1607

Sanders, D. B., \& Mirabel, I. F. 1996, ARA\&A, 34, 749

Schweitzer, M., et al. 2006, ApJ, 649, 79

Schweitzer, M., et al. 2008, ApJ, 679, 101

Siebenmorgen, R., Haas, M., Krügel, E., \& Schulz, B. 2005, A\&A, 436, L5

Skinner, C. J., Smith, H. A., Sturm, E., Barlow, M. J., Cohen, R. J., \& Stacey, G. J. 1997, Nature, 386, 472

Smith, J. D. T., et al. 2007, ApJ, 656, 770

Solomon, P. M., Downes, D., Radford, S. J. E., \& Barrett, J. W. 1997, ApJ, 478, 144

Spoon, H. W. W., Keane, J. V., Cami, J., Lahuis, F., Tielens, A. G. G. M., Armus, L., \& Charmandaris, V. 2005, in IAU Symp. 231, Astrochemistry: Recent Successes and Current Challenges, ed. D. C. Lis, G. A. Blake, \& E. Herbst (Cambridge: Cambridge Univ. Press), 281

Spoon, H. W. W., Keane, J. V., Tielens, A. G. G. M., Lutz, D., \& Moorwood, A. F. M. 2001, A\&A, 365, L353

Spoon, H. W. W., Keane, J. V., Tielens, A. G. G. M., Lutz, D., Moorwood, A. F. M., \& Laurent, O. 2002, A\&A, 385, 1022

Spoon, H. W. W., Koornneef, J., Moorwood, A. F. M., Lutz, D., \& Tielens, A. G. G. M. 2000, A\&A, 357, 898

Spoon, H. W. W., Marshall, J. A., Houck, J. R., Elitzur, M., Hao, L., Armus, L., Brandl, B. R., \& Charmandaris, V. 2007, ApJ, 654, L49

Spoon, H. W. W., et al. 2004, ApJS, 154, 184

Spoon, H. W. W., et al. 2006, ApJ, 638, 759

Staveley-Smith, L., Norris, R. P., Chapman, J. M., Allen, D. A., Whiteoak, J. B., \& Roy, A. L. 1992, MNRAS, 258, 725

Sturm, E., Lutz, D., Tran, D., Feuchtgruber, H., Genzel, R., Kunze, D., Moorwood, A. F. M., \& Thornley, M. D. 2000, A\&A, 358, 481

Sturm, E., et al. 2005, ApJ, 629, L21

Weedman, D. W., \& Houck, J. R. 2009, ApJ, 693, 370

Weedman, D. W., et al. 2005, ApJ, 633, 706

Werner, M. W., et al. 2004, ApJS, 154, 1

Willett, K. W., Darling, J., Spoon, H. W. W., Charmandaris, V., \& Armus, L. 2011, ApJ, 730, 56 (Paper II)

Zakamska, N. L., Gómez, L., Strauss, M. A., \& Krolik, J. H. 2008, AJ, 136, 1607 